\title{
Kingdom of Lesotho: 2008 Article IV Consultation-Staff Report; Staff Supplement; Public Information Notice on the Executive Board Discussion; and Statement by the Executive Director for the Kingdom of Lesotho
}

The following documents have been released and are included in this package:

- $\quad$ The staff report, prepared by a staff team of the IMF, following discussions that ended on November 11, 2008 with the officials of the Kingdom of Lesotho on economic developments and policies. Based on information available at the time of these discussions, the staff report was completed on February 9, 2009. The views expressed in the staff report are those of the staff team and do not necessarily reflect the views of the Executive Board of the IMF.

- $\quad$ A supplement to the staff report.

- $\quad$ A Public Information Notice (PIN) summarizing the views of the Executive Board as expressed during its February 9, 2009, discussion of the staff report that concluded the Article IV consultation.

- $\quad$ A statement by the Executive Director for the Kingdom of Lesotho.

The document(s) listed below will be separately released.

\section{Statistical Appendix}

The policy of publication of staff reports and other documents allows for the deletion of market-sensitive information.

Copies of this report are available to the public from

International Monetary Fund • Publication Services

$70019^{\text {th }}$ Street, N.W. • Washington, D.C. 20431

Telephone: (202) 623-7430 • Telefax: (202) 623-7201

E-mail: publications@imf.org Internet: http://www.imf.org

\section{International Monetary Fund Washington, D.C.}




\section{INTERNATIONAL MONETARY FUND}

\section{KINGDOM OF LESOTHO}

\section{Staff Report for the 2008 Article IV Consultation}

Prepared by the staff representatives for the 2008 consultation with the Kingdom of Lesotho

Approved by Robert Sharer and Anthony Boote

January 23, 2009

- Date: October 29-November 11, 2008. The mission met with Finance Minister Thahane, Central Bank Governor Senaoana, other senior government officials, and representatives of the private sector and the donor community.

- $\quad$ Team: Messrs. Thugge (head), Davoodi, Fontaine, and Torrez (all AFR). Ms. Lephoto (OED) and representatives of the World Bank also attended.

- Lesotho has accepted the obligations of Article VIII, Sections 2, 3, and 4, of the Articles of Agreement and maintains an exchange system free of restrictions on the making of payments and transfers for current international transactions. The Lesotho loti is pegged at par to the South African rand, which is also legal tender in the country.

- Data provision has some shortcomings, but is broadly adequate for surveillance. 


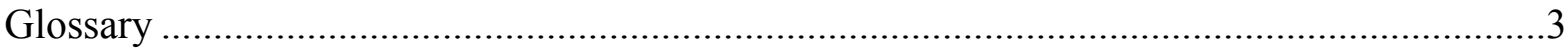

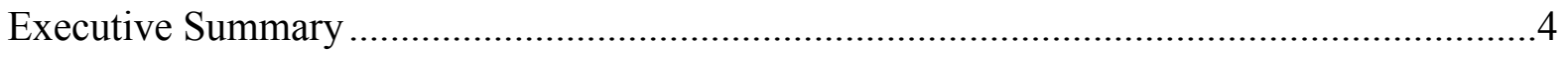

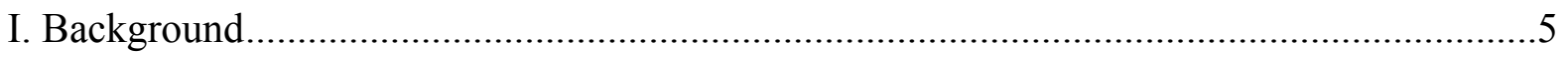

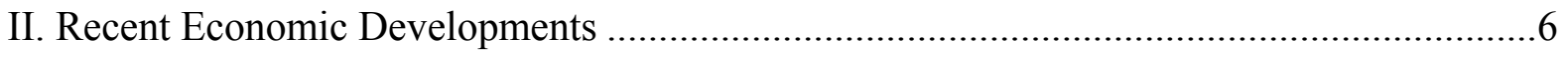

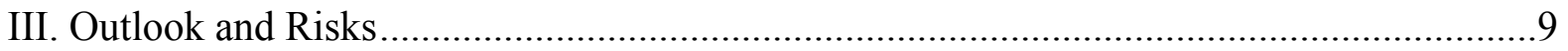

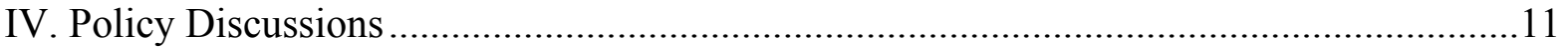

A. Achieving Sustained and Broad-Based Growth.................................................. 12

B. Preserving Fiscal Sustainability and Improving Fiscal Management ....................14

C. Pursuing External Stability........................................................................... 15

D. Ensuring Financial Stability and Development ..............................................18

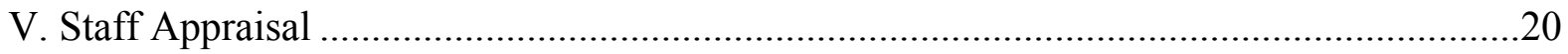

Tables

1. Selected Economic and Financial Indicators, 2004-2013 …...........................................22

2. Central Government Operations, 2005/06-2013/14 ......................................................23

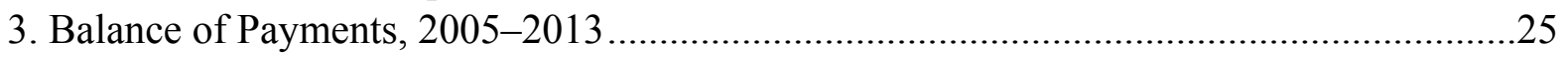

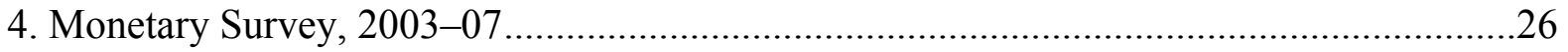

5. Indicators of External Vulnerability, 2003-07 .........................................................2

Figures

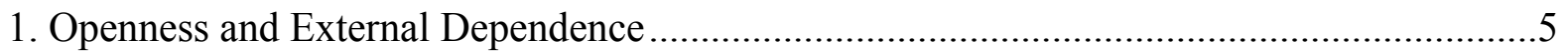

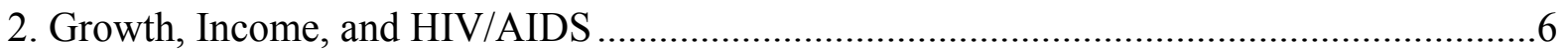

3. Recent Macroeconomic Performance ........................................................................... 7

4. Economic Impact of Lower Diamond and Textile Output .............................................12

Boxes

1. Lesotho's Financial Sector...................................................................................

2. Impact of the Global Financial Crisis and Downturn on Lesotho ..................................... 10

3. Challenges Facing the Textile Sector in Lesotho ......................................................18 


\section{GLOSSARY}

AGOA

CBL

CRP

CMA

DSA

FIA

GSP

IFAD

LHWP

MCC

$\mathrm{M}$

MDGs

NBFIs

PAC

PEM

PPP

PSIRP

RUFIP

SACU

SADC

SACCOs
African Growth and Opportunity Act

Central Bank of Lesotho

Common Revenue Pool

Common Monetary Area

Debt sustainability analysis

Financial Institutions Act

Growth Strategy Paper

International Fund for Agricultural Development

Lesotho Highland Water Project

Millennium Challenge Corporation

Maloti (plural for the Loti, Lesotho's currency)

Millennium Development Goals

Nonbank Financial Institutions

Project Appraisal Committee

Public Expenditure Management

Public-Private-Partnership

Public Sector Improvement and Reform Program

Rural Financial Intermediation Program

Southern African Customs Union

Southern African Development Community

Savings and Credit Cooperatives 


\section{EXECUTIVE SUMMARY}

\section{Background and outlook}

Over the past two years, Lesotho made significant progress in macro-economic performance although implementation of structural reforms has been slow. Lesotho now faces significant risks in the wake of the global financial crisis and economic downturn. Already, the Southern African Customs Union (SACU) receipts, which account for a large part of government revenue, have been revised down significantly; some textile factories have laid off workers owing to reduced demand in the key U.S. market and difficulties securing lines of credit; and one diamond mining company has already suspended operations amid a softening of diamond prices.

With lower SACU revenues, the fiscal surplus is expected to narrow in 2009 and the current account will register a deficit. Economic growth in 2009 is expected to fall below its recent historical average, but should recover in 2010. However, a more protracted global and regional downturn could postpone the timing and limit the size of the recovery.

\section{Key policy issues}

Given the downside risks to SACU revenues, the authorities agreed on the need to strengthen collection of non-SACU revenues, maintain fiscal discipline and improve prioritization of expenditures. They agreed to resuscitate the Project Appraisal Committee to strengthen the process of selection and evaluation of capital projects. They also agreed to form a high-level committee to fast-track implementation of structural reforms that should increase the growth potential, facilitate economic diversity and improve competitiveness.

To contain risks from the weakly-supervised nonbank financial institutions (NBFIs), the authorities agreed to amend the law on credit cooperatives and revise the Financial Institutions Act (FIA). The government has also closed down the operations of the largest Ponzi scheme and is awaiting a court decision to continue with its liquidation. 


\section{BACKGROUND}

\section{Lesotho is a small, highly open, landlocked country, with limited natural} resources, and a narrow production and export base. It has close economic ties with South Africa and its currency, the loti, is pegged to the rand. Aside from subsistence agriculture which employs a majority of the population, the garment sector, transfers from the SACU, ${ }^{1}$ workers' remittances and lately the diamond sector also play an important role in employment, output, and exports (Figure 1). Lesotho is a member of the Common Monetary Area $(\mathrm{CMA})^{2}$ and the Southern African Development Community (SADC).

\section{Figure 1. Openness and External Dependence}
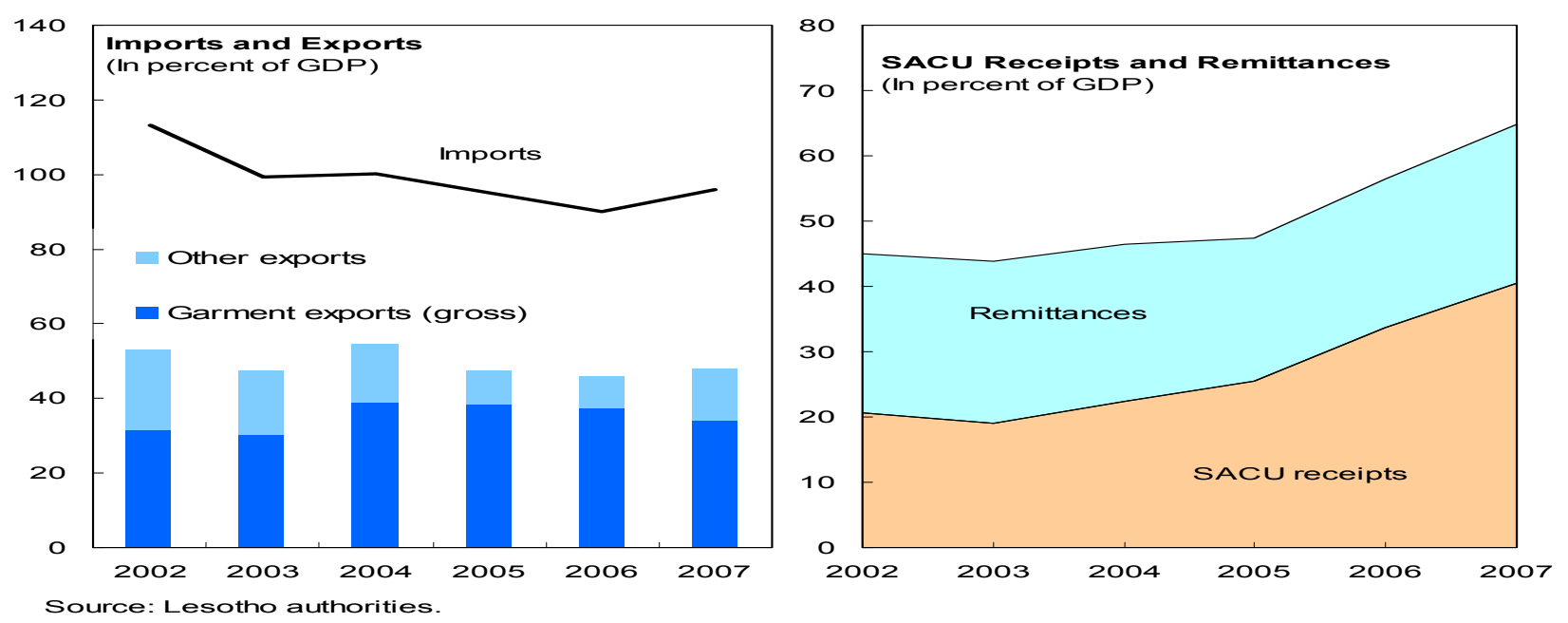

\section{Economic growth has been erratic and social progress has been limited}

(Figure 2). Growth in per capita income averaged about 1 1 $\frac{1}{2}$ percent a year during the period of 1997-2007, well below Lesotho's regional and international comparators. Poverty has declined modestly from 67 percent of households in 1994-95 to 57 percent in 2002-03, and income distribution is highly unequal. In May of this year and in response to the high food prices, the World Food Program began delivering food assistance to the population. It aims to distribute food to 155,000 vulnerable people in Lesotho each year until 2010. The HIV/AIDS epidemic continues to impact negatively on the economy, public service delivery and social development and threatens the achievement of the Millennium Development Goals (MDGs). Already, the country's ranking on the United Nations Human Development Index has deteriorated, falling from the bottom 33 percent of all countries in the early 1990s to the bottom 13 percent in 2007-08.

\footnotetext{
${ }^{1}$ Other members of the SACU are Botswana, Namibia, South Africa, and Swaziland.

${ }^{2}$ CMA consists of SACU members excluding Botswana.
} 
Figure 2. Growth, Income, and HIVIAIDS
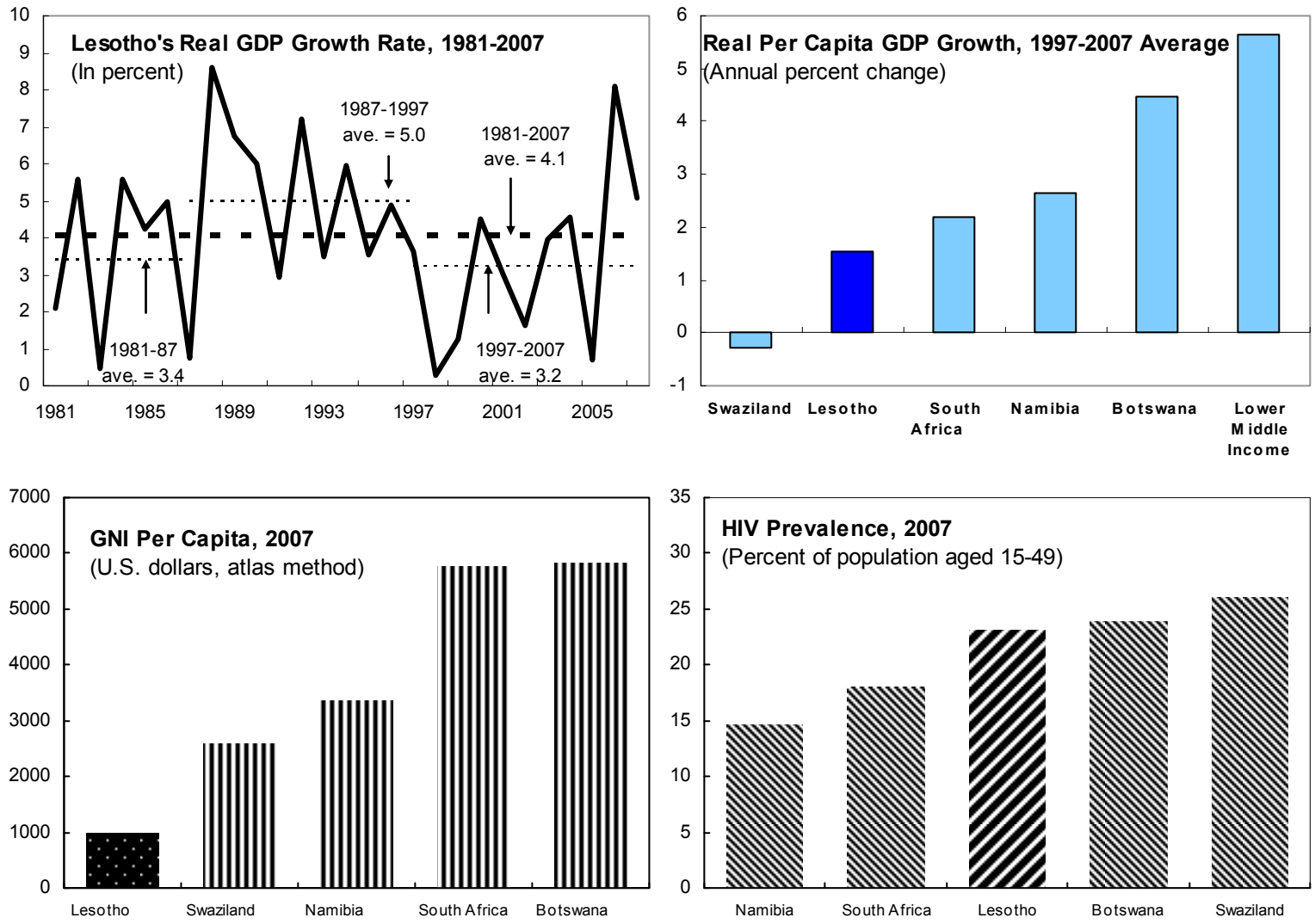

Sources: Lesotho authorities; and the World Bank.

\section{RECENT ECONOMIC DEVELOPMENTS}

\section{Lesotho has made significant progress in macroeconomic performance}

(Figure 3). After a decade of low growth, economic activities surged above historical trends in the last two years, driven by mining, textile and construction sectors. The external current account registered sizable surpluses, reflecting high SACU revenues, workers' remittances, and exports of textiles and diamonds. As a result, gross international reserves was almost US\$1 billion at end-2007

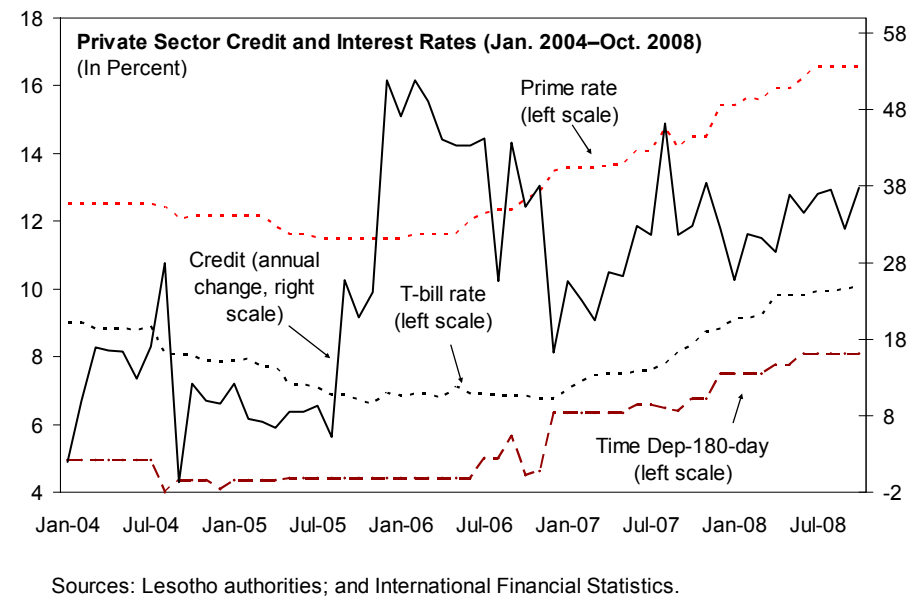
(equivalent to 6.7 months of imports), the highest level in Lesotho's history. Growth in broad monetary aggregates remains high, reflecting the continued accumulation of net foreign assets. The prime lending rate has increased in line with increases in South Africa. 
Figure 3. Recent Macroeconomic Performance
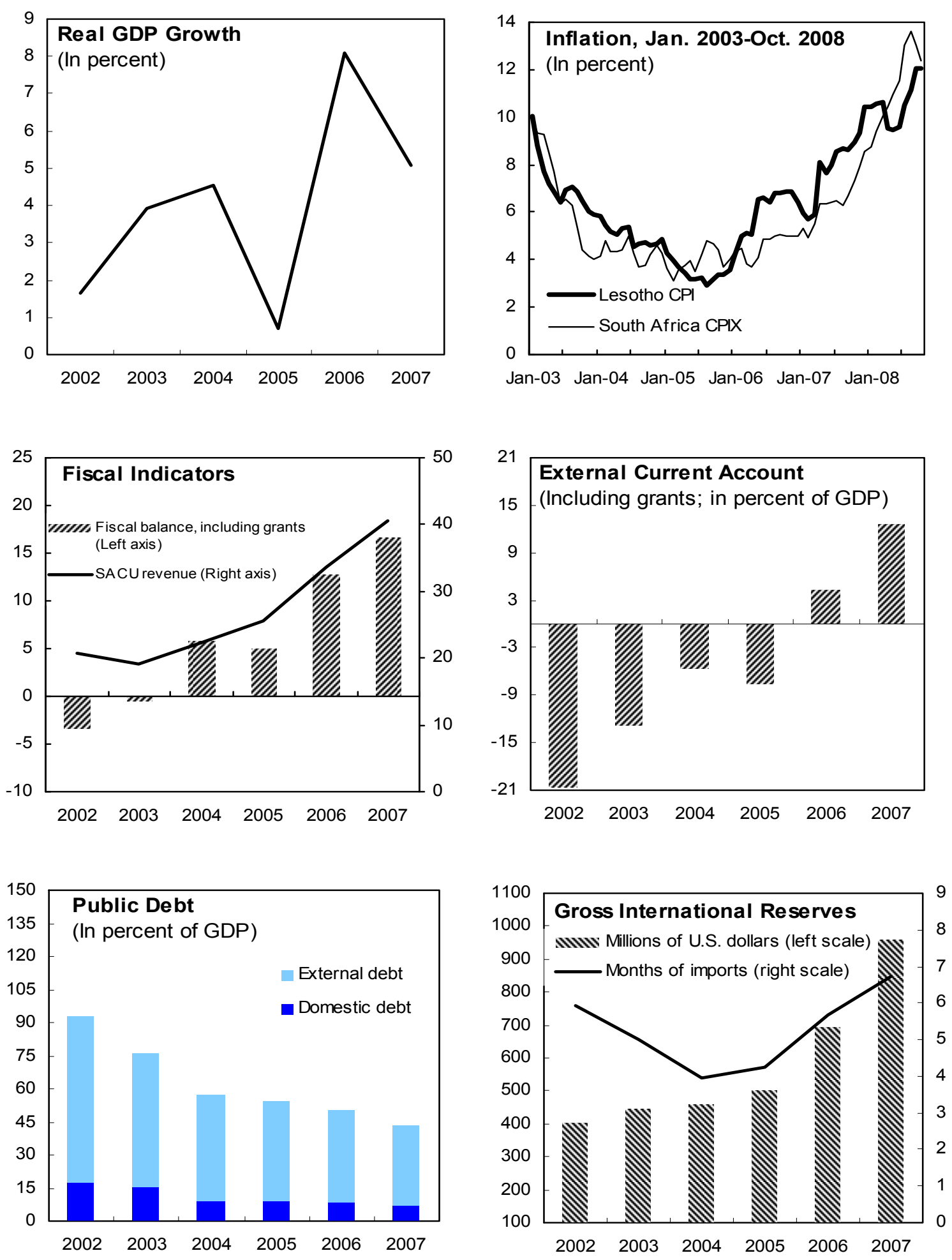

Sources: Lesotho authorities; and IMF staff estimates. 
4. As elsewhere, food and fuel prices have surged in Lesotho, driving the overall inflation up from 6.4 percent at end-2006 to 11.8 percent at end-November 2008.

Lesotho has generally passed-through international fuel and food prices to retail prices, and on April 1, 2008, tariffs for electricity, and public transportation were increased, in line with higher international energy prices and the increase in the price of electricity imported from South Africa. However, in order to boost food production, the 2008/09 budget provides guarantees of (M 105 million) to farmers, to be used for the purchase of inputs, leasing of machinery, and ploughing. The government plans to establish an Agricultural Development Fund over time for continued assistance to farmers. A supplementary budget provides funding (M 40 million) for a well-targeted cash-for-work program that reclaims land due to soil erosion.

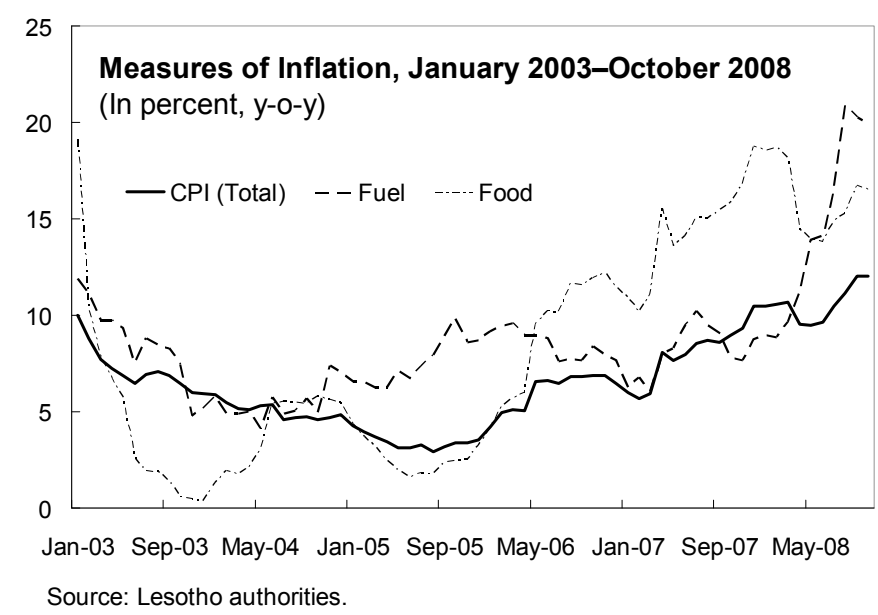

5. Despite a marked increase in development spending, Lesotho recorded its fifth consecutive fiscal surplus in 2007/08 of 10.3 percent of GDP (the highest in the SACU countries) compared with a budgeted deficit of 2.8 percent of GDP. This performance reflected higher-than-budgeted SACU receipts, bolstered by buoyant growth in value-added tax and double digit growth in income taxes, and underspending of both recurrent and development expenditure.

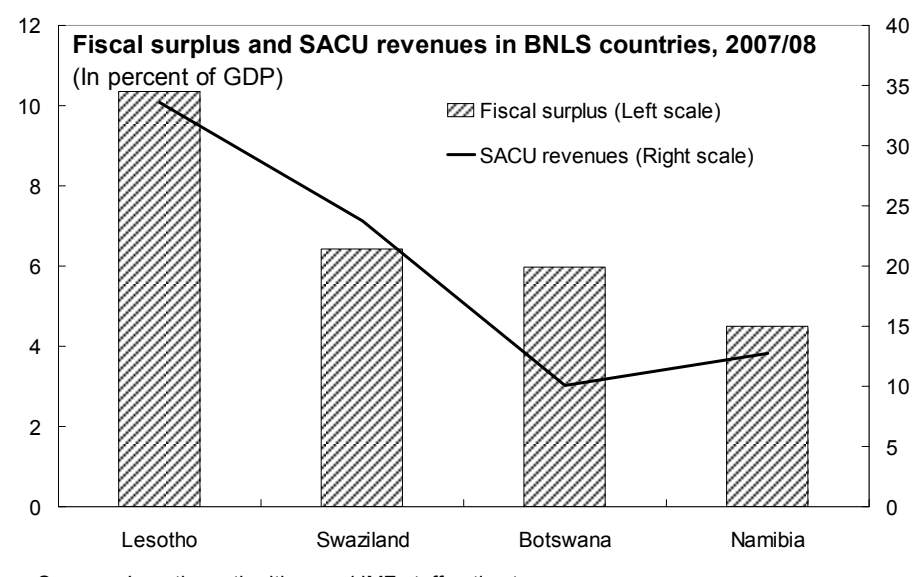

6. Financial sector (Box 1) vulnerabilities stem from the weakly supervised NBFIs, and the emergence of Ponzi schemes. The largest Ponzi scheme (MKM), has been closed but the final resolution of its identified liabilities remains an outstanding policy issue. ${ }^{3}$ On September 23, 2008, the CBL provided to the public a summary of a confidential audit report that indicated that the MKM group is insolvent, with identified assets falling short of investors' original contributions by M300 million (2.7 percent of GDP). ${ }^{4}$ There are so far no

\footnotetext{
${ }^{3}$ NBFIs have not operated any Ponzi schemes.

${ }^{4}$ The MKM's promised payout to investors (including interest) amounts to 9 percent of GDP.
} 
discernible effects on financial intermediation from the collapse of the Ponzi scheme, but many poor individuals have suffered financial losses.

\section{Box 1. Lesotho's Financial Sector}

Lesotho's financial system comprises three South African-majority controlled commercial banks, with total assets equivalent to about 42 percent of GDP. NBFIs, with assets equivalent to 21 percent of GDP, comprise money lenders, insurance companies, private pension funds, unit trusts, and SACCOs. The state-owned Postal Bank provides bank-type services to the unbanked population and micro, small, and medium enterprises, but it is not authorized to make loans. Recently the government established a defined-contribution pension fund for government employees aged 40 years or less.

Assets of the Financial System

\begin{tabular}{|c|c|c|c|c|c|}
\hline & \multirow[b]{2}{*}{$\begin{array}{l}\text { Number of } \\
\text { Institut. }\end{array}$} & \multicolumn{2}{|c|}{ Total Assets as of 2007} & \multirow[b]{2}{*}{$\begin{array}{c}\text { Accept } \\
\text { Deposits }\end{array}$} & \multirow[b]{2}{*}{ Supervised By } \\
\hline & & $\begin{array}{c}\text { In } \\
\text { Maloti Millions }\end{array}$ & $\begin{array}{c}\text { As Percentage } \\
\text { of GDP }\end{array}$ & & \\
\hline Banks & 3 & 4,986 & 42 & Yes & CBL \\
\hline PostBank & 1 & 121 & 1 & Yes & CBL \\
\hline Insurance companies and pension funds 1/ & 5 & 1,958 & 17 & No & CBL \\
\hline Money lenders & 100 & $\ldots$ & $\ldots$ & No & CBL \\
\hline SACCOs $2 /$ & 247 & $\ldots$ & $\ldots$ & Yes & Min. of Trade \& Ind. \\
\hline Investment funds & 3 & 333 & 3 & No & $\mathrm{CBL}$ \\
\hline
\end{tabular}

$1 /$ In addition, there are16 insurance brokers.

2/ There are 1,206 cooperatives, of which 941 are dormant and only 247 are active.

\section{OUTLOOK AND RISKS}

\section{The global financial crisis has adversely affected Lesotho's near-term prospects} (Box 2). Real GDP growth is projected to slow down from 5.1 percent in 2007 to 3.9 percent and 2.1 percent in 2008 and 2009, respectively, owing to lower output in mining and textiles. However, as the global economy recovers, growth is expected to increase to a range of $4-5$ percent in the medium term. The external current account deficit is expected to widen significantly to 8.5 percent of GDP in 2009 owing to reduced diamond and textile exports, remittances, and SACU revenues but then narrow to 5 percent in 2013 in line with the global recovery. Foreign direct investments are also anticipated to decline in 2009, resulting in an overall balance of payments deficit and a reduction in international reserves to 6-7 months of import cover. Balance of payments surpluses are projected beginning 2011, and international reserves are expected to stabilize at about $5 \frac{1}{2}$ months of import cover in the medium term. 


\section{Box 2. Impact of the Global Financial Crisis and Downturn on Lesotho}

Lesotho depends heavily on trade with South Africa and the United States that accounts for 69 percent and 19 percent of its total trade, respectively. The main transmission channels of the crisis include:

SACU Revenues: Slower growth in South Africa's economy reduces import demand, and thus customs duty, excise taxes and the amount of revenues transferred to Lesotho. Following the recent downward revision in South Africa's growth prospects, the projected SACU common revenue pool (CRP) was reduced by 6 percent in 2009 , and by 15 percent in 2010-11. This has led to a significant revision of Lesotho's medium-term fiscal framework.

Textiles. With the slow-down in the U.S. economy, export volumes from Lesotho have declined. In addition, as most of the garment factories are owned by firms in Asia, some of them are facing difficulties obtaining trade credit for input financing from their Asian banks, which have been affected by the global credit crunch.

Diamonds. Weak prices for diamonds and the global credit crunch have resulted in reduced production and exports of diamonds. Already one mining company, Liqhobong, has suspended production and is reassessing its operations, and another, Kao, which is just beginning production, is looking for potential investors - $a$ task that is difficult in the current environment.

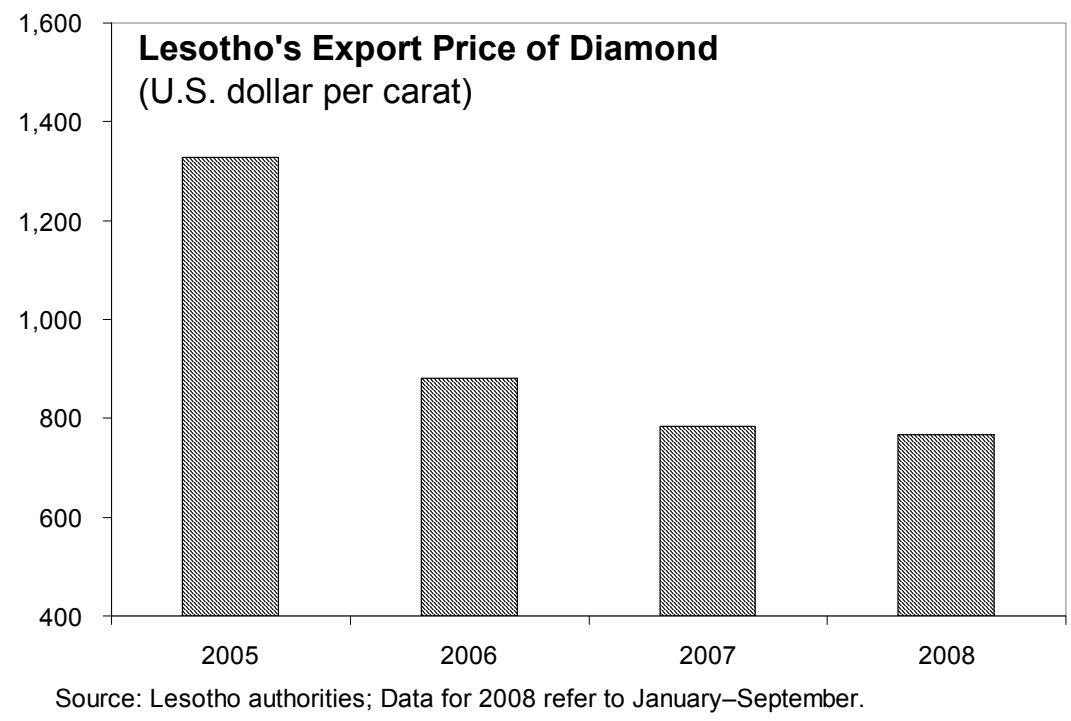

Worker remittances. Remittances from migrant workers, mainly from South Africa, account for about 20 percent of GDP. Already retrenchments in South Africa's mining industry in response to the global credit crunch may put over 9,000 jobs at risk, thus potentially affecting some 50,000 Basothos who work in South Africa's mines. A decline in remittances would reduce private consumption, lower growth and increase poverty. 
Lesotho: Medium-Term Scenario, 2006-2013

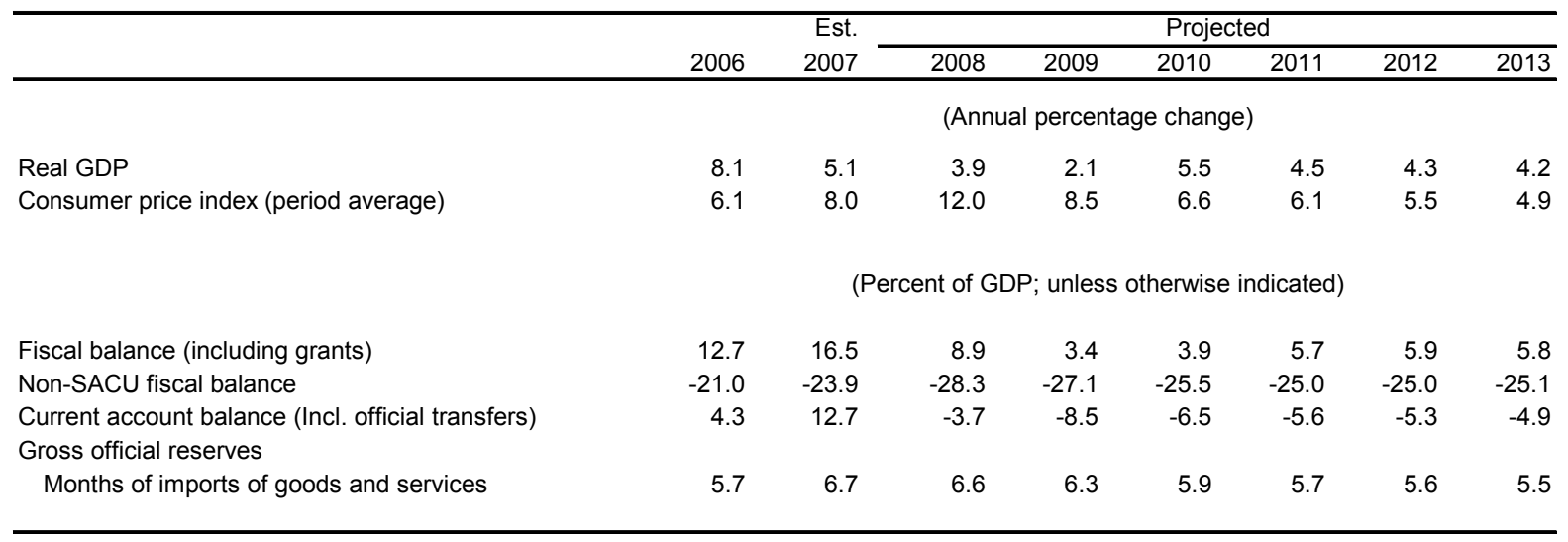

Sources: Lesotho authorities; and IMF staff estimates and projections.

8. Considerable downside risks remain to the baseline outlook. Staff considered the possible impact of a more protracted global financial crisis and economic downturn on Lesotho's economy by assuming a sustained reduction in the production of diamonds and textiles (Figure 4). ${ }^{5}$ The results suggest a need for continuing vigilance on the macroeconomic front, and acceleration of structural reforms to ensure external sustainability. ${ }^{6}$

\section{Policy Discussions}

\section{Against the background of the global financial crisis, the discussions focused on policies to:}

- $\quad$ achieve sustained, broad based growth for poverty reduction;

- $\quad$ preserve fiscal sustainability and improve fiscal management;

- $\quad$ pursue external stability; and

- $\quad$ ensure financial stability and development.

\footnotetext{
${ }^{5}$ The baseline scenario assumes a contraction in mining and textile sectors (of 6- $6 \frac{1}{2}$ percent) followed by a gradual recovery in 2010. The alternative scenario assumes a sustained reduction of 15 percent relative to the baseline scenario.

${ }^{6}$ The impact of lower SACU revenue is considered in the DSA.
} 
Figure 4. Economic Impact of Lower Diamond and Textile Output
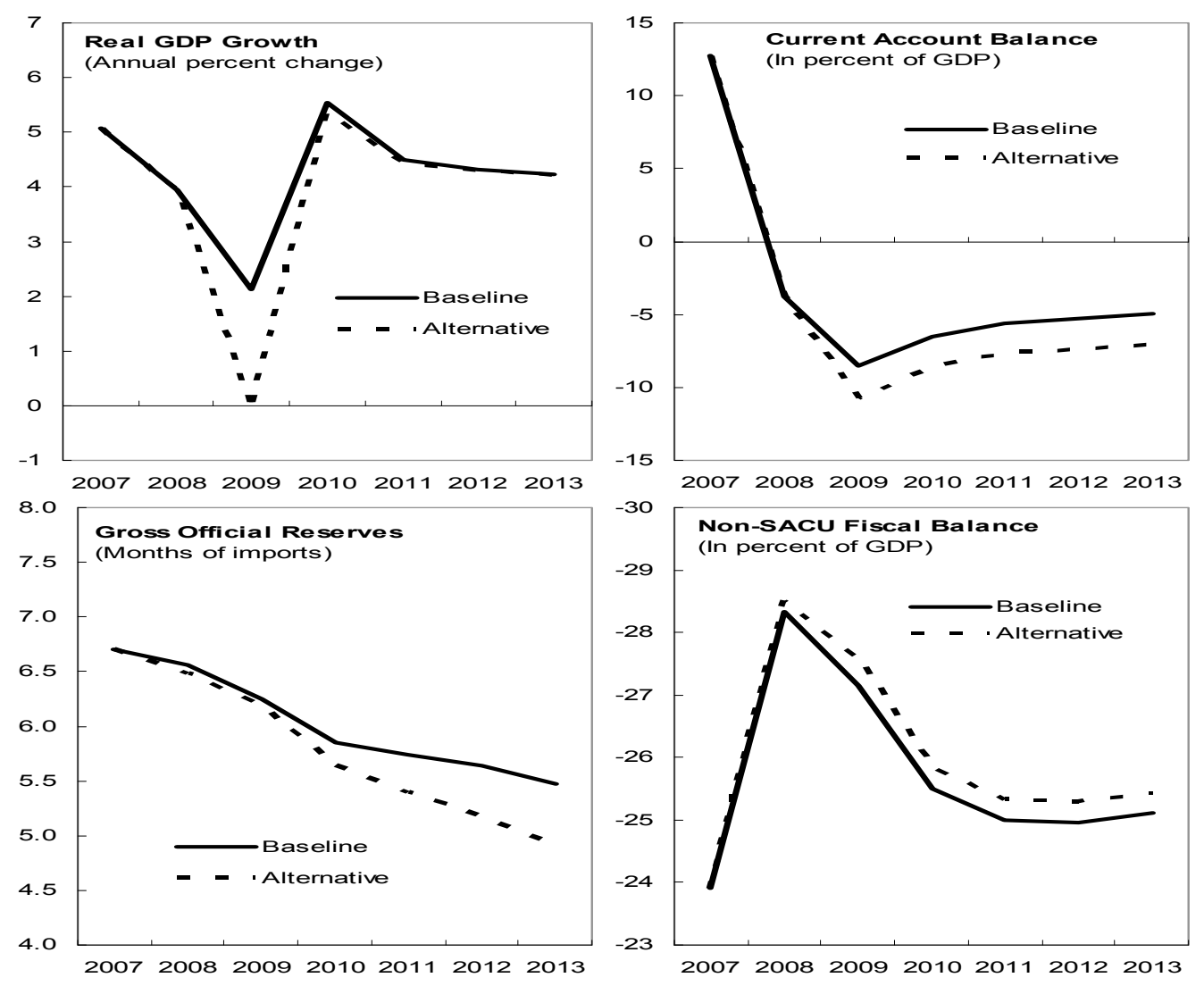

Sources: Lesotho authorities; and IMF staff estimates.

\section{Implementation of Previous Fund Policy Advice}

Fund staff and authorities have generally agreed on Lesotho's main economic challenges. In the past, staff recommended containing recurrent expenditure in light of uncertain SACU revenues, vigorously implementing structural reforms, and taking prompt action to deal with the Ponzi schemes. The authorities' containment of recurrent spending contributed to a substantial surplus in 2007-08, and they closed down the operations of the largest Ponzi scheme. However, the pace of structural reforms has generally been slow mainly due to capacity constraints.

\section{A. Achieving Sustained and Broad-Based Growth}

10. Staff and authorities agreed that accelerating broad-based sustainable economic growth is needed to reduce poverty and inequality, and move toward achieving the MDGs. The recent sharp declines, owing to drought and high cost of inputs, in agricultural production - the source of income for the 70 percent of the rural population - may have exacerbated poverty and worsened income distribution. Staff welcomed, therefore, the government's intention to increase agriculture productivity with the support of the World 
Bank, and to devise a long-term strategy to boost food production. Measures to address the HIV/AIDS pandemic, such as awareness campaigns and increased anti-retroviral therapies, are critical in supporting the development of human capital and increasing productivity in all sectors.

\section{Staff welcomed the focus in the authorities' draft Growth Strategy Paper (GSP)} of identifying binding constraints on growth and improving the investment climate. The GSP rightly emphasizes the importance of prudent macro-fiscal policies, an improved investment climate, enhanced property rights, land reforms, and better infrastructure as linchpins for accelerating pro-poor growth. Staff and the authorities agreed, that for Lesotho to become competitive, it will be critical to expedite the reforms being supported by the World Bank and the Millennium Challenge Corporation (MCC) in such areas as (i) improving the legal, judicial, and regulatory reforms; (ii) raising productivity through improvements in education and health; and (iii) enhancing access to financial services. Staff also noted that Lesotho could take advantage of its proximity to South Africa by deepening regional integration. The recent efforts to establish and operationalize the one-stop-shop are welcomed but closer integration with the relevant ministries is also needed for greater effectiveness.

12. The implementation of projects supported by the MCC and the second phase of Lesotho Highland Water Project (LHWP) should support growth over the next five to ten years. The MCC approved a grant of US\$362.6 million (22 percent of 2007 GDP) under its compact program to assist Lesotho reduce poverty through sustained economic growth. The grant, which came into force in September 2008, has to be utilized within the next five years or Lesotho will lose the balance. The funds will be used to finance projects for provision of water, renovation of health facilities, and development of the private sector. The grant has an administration component for monitoring and evaluation, compact management, and oversight. The second phase of LHWP (62 percent of 2007 GDP) is expected to start in 2012/13 and provides water and electricity to South Africa and Lesotho.

\section{Distribution of MCC grant}
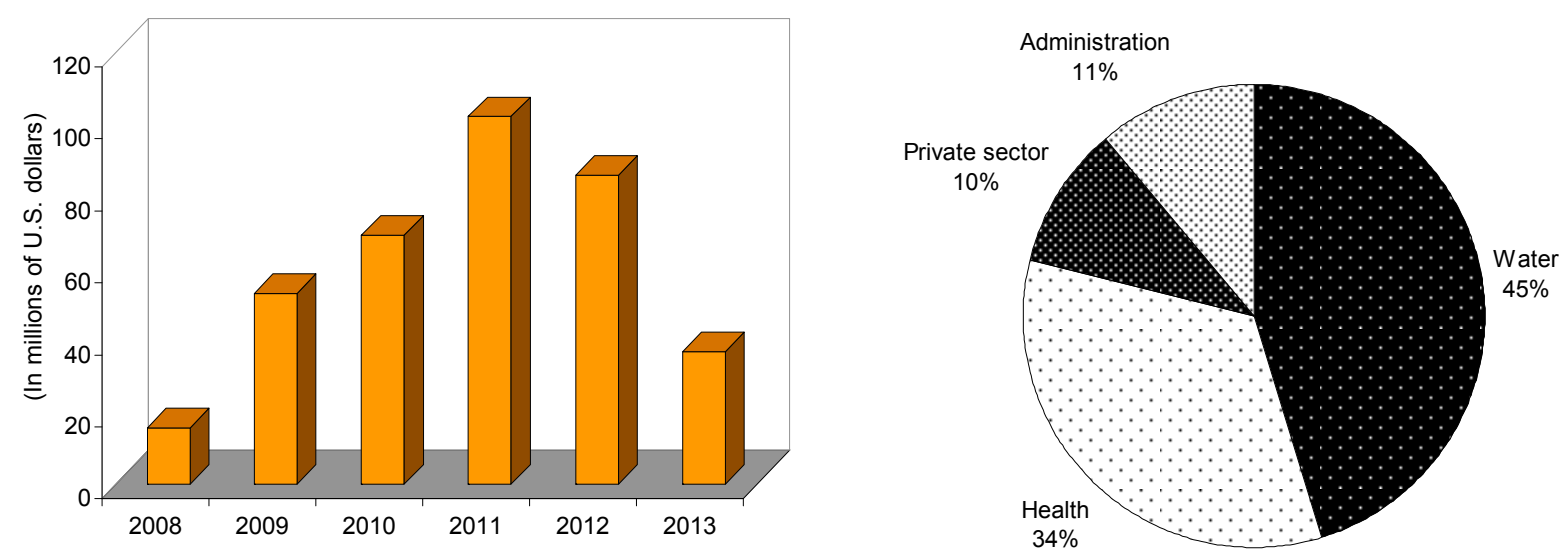
13. The current land tenure system unduly constrains development of agriculture, the financial sector, tourism, and manufacturing. Lack of collaterization of land and clarification of land ownership rights weakens linkages between the agriculture and financial sector while restrictions on foreign ownership and long-term leasing of land reduce incentives for foreign investors to promote tourism and expand factory shells. While agreeing that land reform is important, the authorities noted that progress is likely to be time consuming as it requires consensus-building at the national level.

\section{B. Preserving Fiscal Sustainability and Improving Fiscal Management}

14. The authorities agreed that Lesotho's public finances are vulnerable to downside risks to SACU revenues, which account for about two-thirds of its tax revenues.

Notwithstanding the recent downward revision in the CRP by South Africa, further reduction cannot be ruled out in the event of (i) a potential change in the SACU revenue-sharing formula; (ii) a further slow down in South Africa's economic growth; (iii) a reduction in the common external tariff rates due to trade liberalization; and (iv) the creation of a SADC customs union in 2010, which may change Lesotho's share of SACU revenues.

15. Under unchanged policies and taking into account the authorities' projection for $S A C U$ revenues, the fiscal surplus will narrow from about $81 / 2$ percent of GDP in 2008-09-against a budgeted deficit of 3 percent of GDP - to a surplus of 1.8 percent of GDP in 2009-2010, but then rise as SACU revenues recover. The sharp increase in

Lesotho: Fiscal Framework, 2007/08-2013/14

(In percent of GDP)

\begin{tabular}{|c|c|c|c|c|c|c|c|c|c|}
\hline & \multirow{2}{*}{$\begin{array}{r}\text { Budget } \\
2007 / 08\end{array}$} & \multirow{2}{*}{$\begin{array}{r}\text { Prel. Act. } \\
2007 / 08\end{array}$} & \multirow{2}{*}{$\begin{array}{r}\text { Budget } \\
2008 / 09\end{array}$} & \multicolumn{6}{|c|}{ Projection } \\
\hline & & & & $2008 / 09$ & $2009 / 10$ & $2010 / 11$ & $2011 / 12$ & $2012 / 13$ & $2013 / 14$ \\
\hline Total revenue and grants & 57.2 & 59.3 & 64.4 & 64.0 & 55.3 & 58.3 & 60.2 & 58.7 & 56.5 \\
\hline SACU revenue & 31.8 & 33.6 & 37.6 & 38.4 & 27.9 & 30.0 & 30.9 & 30.9 & 30.9 \\
\hline Non-SACU revenue & 21.2 & 24.2 & 21.7 & 23.4 & 23.3 & 23.2 & 23.2 & 23.2 & 23.2 \\
\hline Grants & 4.1 & 1.5 & 5.1 & 2.2 & 4.1 & 5.1 & 6.0 & 4.6 & 2.4 \\
\hline $\begin{array}{l}\text { Total expenditure and net lending } \\
\text { Of which: }\end{array}$ & 59.9 & 48.9 & 67.4 & 55.5 & 53.5 & 53.8 & 54.1 & 52.9 & 50.7 \\
\hline Current expenditure & 44.1 & 38.5 & 51.5 & 44.7 & 39.9 & 39.6 & 39.0 & 38.5 & 38.1 \\
\hline Wages and salaries & 14.5 & 13.4 & 15.8 & 13.7 & 13.5 & 13.4 & 13.3 & 13.2 & 13.2 \\
\hline Goods and services & 14.3 & 11.5 & 16.9 & 13.7 & 13.7 & 13.7 & 13.7 & 13.7 & 13.7 \\
\hline Transfers and subsidies & 12.2 & 11.2 & 17.7 & 16.5 & 11.9 & 11.7 & 11.3 & 10.9 & 10.6 \\
\hline Capital expenditure & 16.0 & 10.6 & 16.0 & 10.9 & 13.6 & 14.3 & 15.1 & 14.4 & 12.7 \\
\hline Overall balance & -2.8 & 10.3 & -3.0 & 8.5 & 1.8 & 4.5 & 6.1 & 5.9 & 5.8 \\
\hline Financing & 2.8 & -8.3 & 3.0 & -8.5 & -1.8 & -4.5 & -6.1 & -5.9 & -5.8 \\
\hline Statistical discrepancy & 0.0 & 2.0 & 0.0 & 0.0 & 0.0 & 0.0 & 0.0 & 0.0 & 0.0 \\
\hline Memorandum item: & & & & & & & & & \\
\hline Non-SACU fiscal balance & -34.6 & -23.3 & -40.6 & -29.9 & -26.0 & -25.5 & -24.8 & -25.0 & -25.1 \\
\hline
\end{tabular}


transfers in 2008-09 reflects full capitalization of the pension fund. In 2009-10 and beyond, non-SACU revenues are assumed to grow in line with nominal GDP. On the expenditure side, having already introduced a significant pension reform in the 2008-09 budget, which recognized the contingent liability ( $4 \frac{1}{2}$ percent of GDP) associated with the existing unfunded Defined Benefit Plan, the authorities do not intend to introduce any major expenditure policy changes. The wage bill, which is already one of the highest in subSaharan Africa, is expected to decline gradually in line with the implementation of the Public Sector Improvement and Reform Program (PSIRP). Over the medium term, the overall fiscal position is projected to remain in surplus, consistent with a stable non-SACU deficit (the overall fiscal balance excluding SACU revenues) of about 24-25 percent of GDP and a substantial reduction in the domestic debt-to-GDP ratio.

\section{Looking ahead, the fiscal policy challenge will be to respond effectively to a} possible further deterioration in SACU revenues. To mitigate against that risk, the government needs to strengthen non-SACU revenues and contain overall expenditures while at the same time shifting the allocation of resources toward development outlays that support growth. Implementation of these measures would permit a lower non-SACU deficit than projected under the unchanged policy scenario. Staff urged the government to avoid a further general wage increase and limit future recruitment to high priority areas. The authorities agreed that the current wage policy may not be sustainable, and emphasized that full implementation of the PSIRP remains a government priority.

\section{Staff noted the government's intention to improve the public expenditure} management (PEM) system and resuscitate the Project Appraisal Committee (PAC) to improve productivity of public investment. The staff and the authorities agreed that the persistent underspending of the budget (10 percent of GDP in 2007/08) reflects primarily the formulation of budget on the basis of the previous year's budget appropriation rather than outturn. To address this problem, the authorities agreed to formulate future budgets, starting with the 2009/10 budget, on the basis of the expected outturn. They also intend to embark on staff training in order to remove capacity bottlenecks and fast-track the implementation of the new Integrated Financial Management Information System. A monitoring and reporting framework will also be considered with a view to enhancing accountability in the use of resources. The authorities are funding two investment projects under a Public-PrivatePartnership (PPP). They are aware of the risks and challenges of PPPs and are monitoring their implementation in collaboration with the PAC. Staff urged that an appraisal framework be put in place to vet new PPP projects and thereby avoid assuming excessive contingent liabilities.

\section{Pursuing External Stability}

18. Economic policies have been consistent with external stability. The staff agreed with the authorities that the current exchange rate system should be maintained as it facilitates capital and current transactions with the country's most important economic 
partner, South Africa. The main policy focus should be on fast-tracking the implementation of key structural reforms in order to enhance productivity and competitiveness.

19. The assessment of the level of the real exchange rate was based on three approaches, the purchasing power parity approach adjusted for productivity differentials, the external sustainability approach, and the macro balance approach. ${ }^{7}$ Based on information through September 2008, the approaches suggested an overvaluation in the range of 5-11 percent. However, this has largely been offset since then by the depreciation of the loti by 13 percent through end-2008. Caution should be exercised in reaching a conclusion given weaknesses in data and the period of assessment. However, Lesotho's skewed trade pattern, with imports (mostly from South Africa) far exceeding exports (mainly to the United States), complicates the interpretation and usefulness of the standard real effective exchange rate for measuring competitiveness.
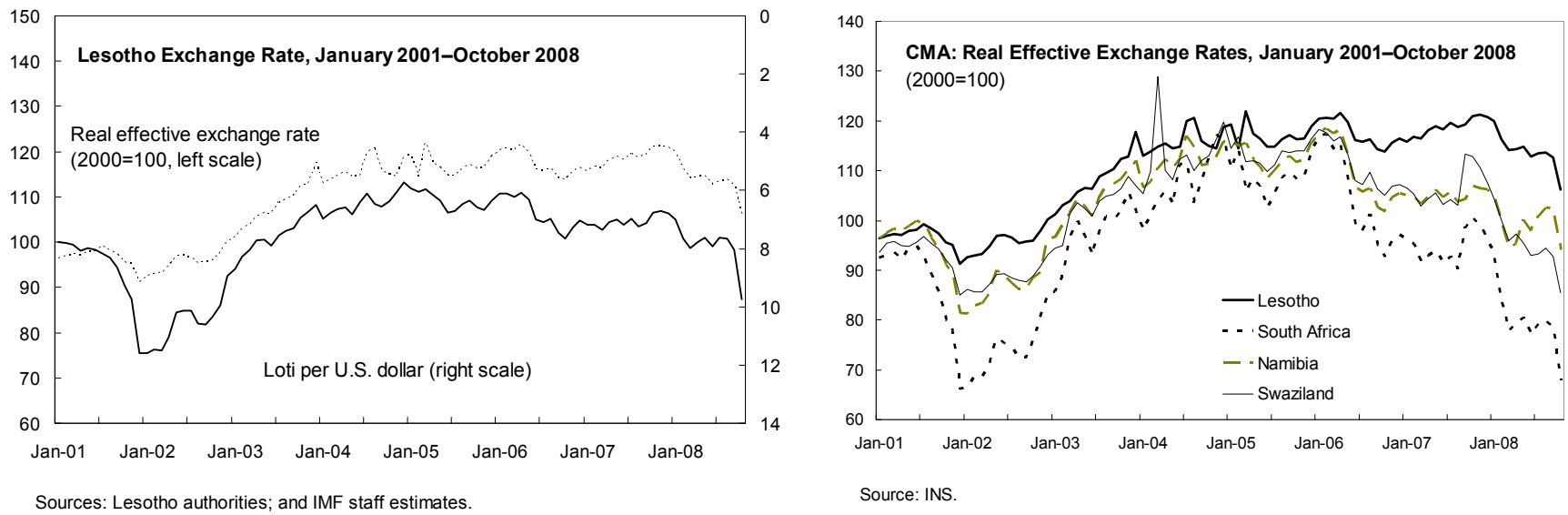

20. Lesotho scores poorly internationally and regionally on most measures of the cost of doing business that are critical to enhancing its competitiveness. Already the government has extended financial support to one large textile company to prevent layoffs and other problems are emerging within the textile sector (Box 3). The current account deficit will widen further to $8 \frac{1}{2}$ percent of GDP in 2009 from 3.7 percent of GDP in 2008; and exports of textiles will fall throughout the projection period. Given Lesotho's real effective exchange rate is largely exogenous in light of the peg of the loti to the rand and with inflation closely tracking that of South Africa, the sustainability of the current account therefore depends crucially on pursuing competitiveness through rigorous implementation of structural reforms.

\footnotetext{
${ }^{7}$ These approaches and the key underlying assumptions are given in two 2007 IMF country reports on Lesotho (Reports no.08/135 and no.08/136).
} 


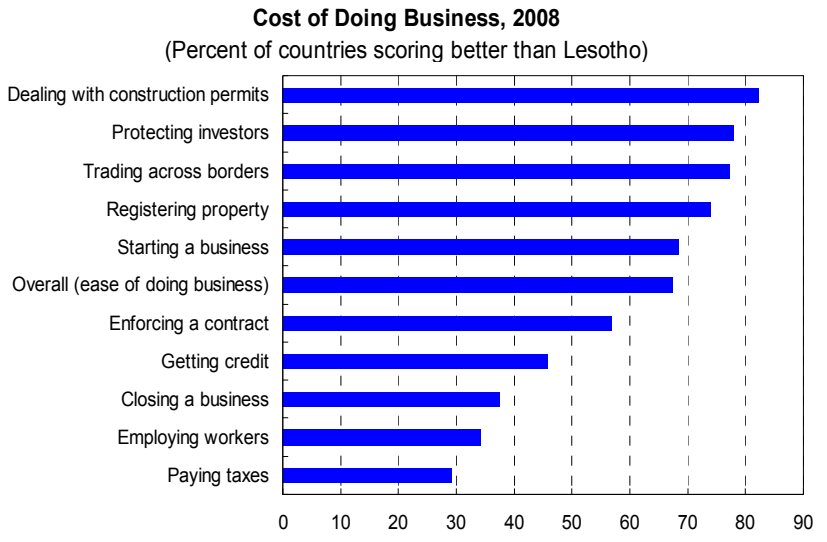

Source: World Bank's Doing Business (2009).

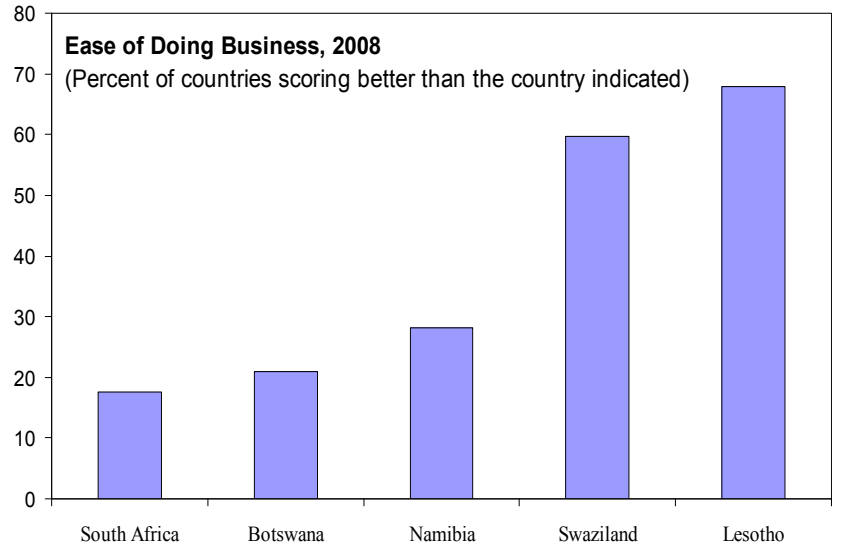

21. The authorities agreed that a continued build-up of international reserves depends in large part on the pursuit of a prudent fiscal stance despite declining SACU transfers. This is particularly important given the falling level of exports (textiles and diamonds), and remittances. The authorities noted that their net international reserve, currently twice the target set by the CBL to support the exchange rate peg, is adequate, and that they would take appropriate measures, if needed, to ensure that objective is sustained.

Indicators of Reserve Adequacy at End-2007

(In percent unless otherwise indicated)

\begin{tabular}{lccc}
\hline & \multicolumn{3}{c}{ Ratio of International Reserve to: } \\
\cline { 2 - 4 } & $\begin{array}{c}\text { Imports of Goods and } \\
\text { Services (In Months) }\end{array}$ & GDP & Broad Money \\
\hline Botswana & 28.0 & 78.6 & 181.4 \\
Lower middle income countries & 10.5 & 33.9 & $\ldots$ \\
Lesotho & 6.7 & $\mathbf{5 7 . 8}$ & 162.4 \\
Sub-Saharan Africa (SSA) & 5.7 & 16.9 & 37.2 \\
Low income countries & 5.5 & 16.6 & $\ldots$ \\
South Africa & 3.7 & 10.6 & 12.3 \\
Swaziland & 3.5 & 25.3 & 94.4 \\
Nonresource intensive SSA & 3.8 & 12.0 & 18.3 \\
Namibia & 2.8 & 10.7 & 20.1 \\
\hline
\end{tabular}

Sources: World Economic Outlook; AFR's Regional Outlook; and staff estimates.

22. With regard to external debt, the staff analysis shows that Lesotho's public and publicly guaranteed debt is sustainable, although there is a moderate risk of debt distress (see the Debt Sustainability Analysis Annex). There is therefore a need to continue pursuing prudent debt policies, including seeking loans on highly concessional terms. 


\section{Box 3. Challenges Facing the Textile Sector in Lesotho}

Not since the expiration of the Multi Fiber Agreement on January 1, 2005 has there been so much pressure on the textile sector in Lesotho. With about two-thirds of its exports going to the United States, the industry has been hit hard by the recent downturn in the U.S. economy. Garment exports to the United States during the first 10 months of 2008 are down by 15 percent compared with the same period in 2007 and Lesotho's share of the U.S. market has fallen.

Among the many other challenges facing the sector is the unavailability of credit from Asia and the pending expiration of the third-country provision for sourcing inputs under the African Growth and Opportunity Act (AGOA) by 2012. This is of particular importance considering that Lesotho has a limited number of fabric mills and will have difficulty sourcing inputs from within the region. The sector is also weighed down by high labor and shipping costs, a shortage of factory shells, and the lack of basic infrastructure, such as water, communications and electricity services.

Lesotho: Textile Exports and Employment 2002-08

\begin{tabular}{lrrrrrrr}
\hline & 2002 & 2003 & 2004 & 2005 & 2006 & 2007 & 2008 \\
\hline & & & & & & & \\
Textiles (in millions of U.S. dollars) & 211.8 & 301.5 & 501.3 & 527.7 & 567.2 & 569.9 & 454.4 \\
Percent of total exports & 59.7 & 64.1 & 71.4 & 81.1 & 81.1 & 70.9 & 63.8 \\
Employment 1/ & 34,125 & 46,635 & 45,034 & 40,813 & 41,535 & 41,149 & 40,939 \\
& & & & & & &
\end{tabular}

Sources: Central Bank of Lesotho; and Lesotho National Development Corporation.

1/ Employment is for end-September 2008.

\section{Responding to the challenges}

Going forward, the challenge is to achieve international competitiveness in the face of strong expansion of Asian exports to their traditional export market in the United States, especially after 2008, when the bilateral agreements restricting Chinese exports expire. In spite of its relatively high unit labor costs compared to those of the Asian textile producing countries and longer turn-around time, trade preferences have helped Lesotho. However, there are no guarantees that the current benefits under the AGOA will last beyond 2012. Moreover, possible reductions in most-favored-nation tariffs in industrial countries under the current Doha Round of multilateral trade negotiations, could further reduce the preference margin for African exports, and adversely affect Lesotho's garment sector. It is essential, therefore, that key structural reforms be implemented quickly if Lesotho is to become competitive.

\section{Ensuring Financial Stability and Development}

\section{The banking sector seems to have weathered well the current global financial} crisis thus far. The three largely South-African-owned commercial banks continue to be well capitalized, liquid and profitable. However, there has been a slight increase in nonperforming loans, although these are still moderate and are fully provisioned. Some vulnerability may remain as bank's loan portfolios continue to be concentrated on a few borrowers. Staff welcomed the approval by Parliament of the Anti-Money Laundering 
legislation and urged that the necessary regulations be issued and the Financial Intelligence Unit be put in place as quickly as possible.

Lesotho: Commercial Banks' Quarterly Performance Ratios, 2006-08

\begin{tabular}{|c|c|c|c|c|c|c|c|c|c|c|c|}
\hline & \multicolumn{4}{|c|}{2006} & \multicolumn{4}{|c|}{2007} & \multicolumn{3}{|c|}{2008} \\
\hline & Mar. & Jun. & Sep. & $\overline{\text { Dec. }}$ & Mar. & Jun. & Sep. & Dec. & Mar. & Jun. & Sep. \\
\hline \multicolumn{12}{|l|}{ Performance ratios } \\
\hline \multicolumn{12}{|l|}{ Capital adequacy } \\
\hline Basel capital ratio & 25.0 & 23.0 & 19.0 & 19.0 & 20.0 & 17.0 & 17.0 & 14.0 & 19.0 & 16.0 & 15.0 \\
\hline Total capital to assets & $\ldots$ & $\ldots$ & $\ldots$ & $\ldots$ & 0.1 & 7.5 & 8.2 & 8.1 & 7.7 & 7.2 & 7.9 \\
\hline \multicolumn{12}{|l|}{ Nonperforming loans net of provisions } \\
\hline to total capital & 2.0 & 2.0 & 6.0 & 6.0 & 9.0 & 6.6 & 0.1 & 0.2 & -0.8 & -0.6 & 3.1 \\
\hline Top 20 exposures to total capital & 84.0 & 63.0 & 98.0 & 117.0 & 116.0 & 148.7 & 143.3 & 124.4 & 124.8 & 132.6 & 139.3 \\
\hline \multicolumn{12}{|l|}{ Asset quality } \\
\hline Loans to deposits ratio & 30.0 & 17.0 & 30.0 & 26.0 & 26.0 & 29.4 & 31.3 & 28.7 & 31.4 & 30.0 & 34.6 \\
\hline Earning assets to total assets & 94.0 & 95.0 & 94.0 & 95.0 & 95.0 & 93.3 & 96.0 & 93.6 & 102.7 & 97.0 & 91.5 \\
\hline Nonperforming loans to total loans & 1.0 & 2.0 & 2.0 & 2.0 & 3.0 & 3.4 & 1.7 & 1.7 & 1.5 & 1.7 & 3.5 \\
\hline Reserve for losses to total loans & 2.0 & 3.0 & 2.0 & 3.0 & 3.0 & 2.6 & 2.6 & 2.6 & 2.6 & 3.2 & 3.8 \\
\hline Reserve for losses to nonperforming loans & 242.0 & 161.0 & 112.0 & 125.0 & 93.0 & 77.8 & 147.9 & 152.4 & 174.8 & 190.5 & 107.8 \\
\hline \multicolumn{12}{|l|}{ Liquidity ratios } \\
\hline Liquid assets to total deposits & 112.0 & 132.0 & 88.0 & 100.0 & 98.0 & 96.4 & 96.8 & 92.9 & 101.4 & 102.7 & 101.3 \\
\hline Available reserves to total deposits & 6.0 & 6.0 & 5.0 & 4.0 & 5.0 & 7.7 & 4.3 & 5.3 & 2.0 & 3.3 & 7.7 \\
\hline Liquid assets to total assets & 75.0 & 83.0 & 75.0 & 78.0 & 78.0 & 75.5 & 74.4 & 74.1 & 75.6 & 76.9 & 74.0 \\
\hline Current assets to total current liabilities & 85.0 & 95.0 & 83.0 & 85.0 & 84.0 & 81.6 & 81.0 & 81.5 & 87.7 & 82.9 & 80.4 \\
\hline \multicolumn{12}{|l|}{ Profitability ratios $1 /$} \\
\hline \multicolumn{12}{|l|}{ Net income to average total } \\
\hline assets (return on assets) & 1.0 & 1.0 & 2.0 & 2.0 & 1.0 & 1.2 & 1.8 & 2.6 & 0.8 & 1.6 & 2.4 \\
\hline \multicolumn{12}{|l|}{ Net income to average total } \\
\hline equity (return on equity) & 7.0 & 8.0 & 20.0 & 27.0 & 8.0 & 14.6 & 21.8 & 31.6 & 10.4 & 21.8 & 31.7 \\
\hline Net interest margin & & & & & 0.0 & 2.7 & 3.8 & 5.5 & 1.7 & 3.3 & 5.0 \\
\hline Total expenses to total income & 75.0 & 73.0 & 68.0 & 70.0 & 74.0 & 74.8 & 72.1 & 71.3 & 71.0 & 69.4 & 68.6 \\
\hline \multicolumn{12}{|l|}{ Solvency ratios } \\
\hline Total debt ratio & 12.0 & 12.0 & 10.0 & 7.0 & 8.0 & 7.5 & 8.2 & 8.1 & 8.2 & 7.2 & 7.9 \\
\hline
\end{tabular}

Source: Central Bank of Lesotho.

$1 /$ Since 2005 , affected by the operations of two new banks.

24. There continues to be a need to increase access to financial services for a large proportion of the underserved population including for small and medium-term enterprises. Staff welcomed the efforts under way supported by the MCC and the International Fund for Agricultural Development (IFAD) under the Rural Financial Intermediation Program (RUFIP) to increase access to financial

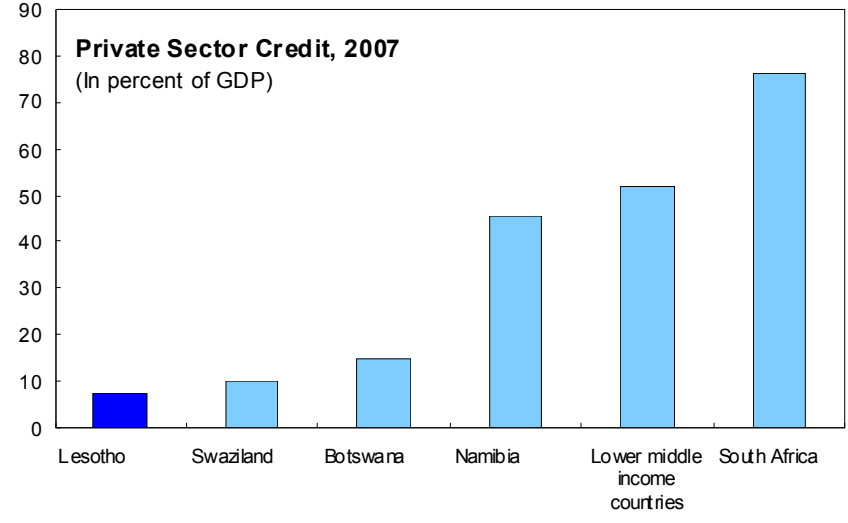

Sources: World Economic Outlook; and International Financial Statistics. services, including by strengthening the institutional and operational framework of PostBank. Implementation of these reforms should deepen and enhance the efficiency of the financial sector. 
25. The extent of the operation of the SACCOs, which by law are permitted to take deposits from nonmembers, is not well known, and the quality of regulatory oversight, performed by the Ministry of Trade, is limited. The authorities intend to repeal Article 68 of the Cooperative Societies Act of 2000 that allows deposit mobilization from nonmembers and may consider converting large SACCOs into commercial banks and placing them under the supervision of the CBL. They are also reviewing the legislation on NBFIs under the IFAD's RUFIP, and more broadly, intend to further strengthen the CBL's supervisory and regulatory authority of the financial sector and prevent emergence of new Ponzi-type schemes.

\section{Staff Appraisal}

26. After two years of good performance, Lesotho's economy now faces significant downside risks owing to the international financial crisis. Projections for SACU revenues have been revised downward significantly and demand for Lesotho's garments has fallen, as has the price of diamonds. The authorities should therefore fast track structural reforms and safeguard fiscal sustainability over the medium term.

27. Broad-based growth will require prioritizing and implementing the reforms supported by the World Bank and the MCC. The government's growth diagnostic study correctly recognizes a number of key constrains that hold growth in check, but it needs to prioritize and remove the key cross-cutting constraints. Of particular importance is land reform, which could help deepen the financial sector and serve as a catalyst for reinvigorating other sectors, such as tourism and manufacturing which are needed for sustaining growth and poverty reduction.

28. Structural reforms need to be accelerated. Consolidating the recent growth performance also requires determined effort to fast-track the implementation of other reforms to reduce the cost of doing business, improve the investment climate, and increase investment in human and physical capital. Implementing these reforms should also help diversify the economy, increase competitiveness and thereby help achieve external sustainability. To ensure implementation and close monitoring, staff recommends charging a high-level committee with the task of accelerating structural reforms, an approach that has worked in other countries.

\section{An appropriate fiscal policy strategy to mitigate risks from lower SACU}

revenues should be put in place. It could include: (i) strengthening non-SACU revenues; (ii) containing recurrent spending by avoiding a general wage increase so as to create fiscal space for increased public investment; (iii) improving the entire process of selecting, monitoring, evaluating, and reporting progress on capital investment projects; (iv) making the budget a more effective tool for public policy by formulating it on an outturn basis rather than on unrealistic budget appropriations that do not advance transparency, accountability, and effective use of scarce resources; and (v) accelerating the civil service reform component 
of the PSIRP, including by linking wage increases to performance and to qualified professionals within the health and education sectors.

30. Staff commends the authorities on their recent decision to close the largest Ponzi scheme and notes that an early successful resolution would serve the country well. It would allow regulators to focus on their core task of ensuring an efficient functioning of the formal financial sector that enhances access to finance and mobilizes resources for productive investment.

31. Prompt action is needed to address vulnerabilities in NBFIs. The amendments to the FIA to strengthen the supervision and regulatory role of the CBL over NBFIs need to be expedited. Staff therefore welcomes the intention to repeal Article 68 of the Cooperative Societies Act of 2000 that allows deposit mobilization from nonmembers.

32. It is proposed that the next Article IV consultation be held on the standard 12-month cycle. 
Table 1. Lesotho: Selected Economic and Financial Indicators, 2004-2013 1/

\begin{tabular}{|c|c|c|c|c|c|c|c|c|c|c|}
\hline & \multirow[b]{2}{*}{2004} & \multirow[b]{2}{*}{2005} & \multirow[b]{2}{*}{2006} & \multirow{2}{*}{$\begin{array}{r}\text { Est. } \\
2007\end{array}$} & \multicolumn{6}{|c|}{ Projections } \\
\hline & & & & & 2008 & 2009 & 2010 & 2011 & 2012 & 2013 \\
\hline & \multicolumn{10}{|c|}{ (Annual percentage change; unless otherwise indicated) } \\
\hline \multicolumn{11}{|l|}{ National income and prices } \\
\hline Real GDP & 4.6 & 0.7 & 8.1 & 5.1 & 3.9 & 2.1 & 5.5 & 4.5 & 4.3 & 4.2 \\
\hline Real GNP & 2.2 & 0.0 & 10.7 & 5.0 & 4.7 & -2.0 & 4.2 & 3.8 & 3.9 & 4.0 \\
\hline Consumer price index (period average) & 5.0 & 3.4 & 6.1 & 8.0 & 12.0 & 8.5 & 6.6 & 6.1 & 5.5 & 4.9 \\
\hline Consumer price index (end of period) & 5.0 & 3.5 & 6.4 & 10.5 & 13.9 & 8.2 & 7.0 & 6.6 & 5.4 & 4.9 \\
\hline GDP (millions of maloti) & 8,332 & 8,750 & 10,269 & 11,778 & 13,418 & 14,899 & 17,012 & 18,741 & 20,523 & 22,203 \\
\hline GNP (millions of maloti) & 10,240 & 10,683 & 12,844 & 14,722 & 16,898 & 17,998 & 20,298 & 22,224 & 24,244 & 26,178 \\
\hline \multicolumn{11}{|l|}{ External sector } \\
\hline Exports, f.o.b. 2/ & 49.2 & -7.3 & 7.5 & 14.9 & -11.4 & -8.9 & 1.5 & -0.3 & -0.7 & -0.2 \\
\hline Imports, f.o.b. 2/ & 31.0 & 1.0 & 4.6 & 17.3 & 5.2 & -5.9 & 2.0 & 3.5 & 2.6 & 2.3 \\
\hline Nominal effective exchange rate 3 / & 0.5 & -1.8 & -3.6 & 0.6 & $\ldots$ & $\ldots$ & $\cdots$ & $\ldots$ & $\cdots$ & $\ldots$ \\
\hline Real effective exchange rate $3 /$ & 0.7 & 0.0 & -2.2 & 3.6 & $\ldots$ & $\ldots$ & $\ldots$ & $\ldots$ & $\ldots$ & $\ldots$ \\
\hline \multicolumn{11}{|l|}{ Money and credit } \\
\hline Net foreign assets 4/ & 22.4 & 12.4 & 73.2 & 66.0 & $\cdots$ & $\cdots$ & $\cdots$ & $\cdots$ & $\cdots$ & $\ldots$ \\
\hline Net domestic assets 4/ & -19.1 & -3.3 & -37.9 & -49.7 & $\ldots$ & $\ldots$ & $\ldots$ & $\ldots$ & $\ldots$ & $\ldots$ \\
\hline Credit to the government & -23.7 & -8.3 & -22.7 & -52.0 & $\ldots$ & $\ldots$ & $\ldots$ & $\ldots$ & $\ldots$ & $\ldots$ \\
\hline Credit to the rest of the economy & 2.5 & 10.3 & 4.3 & 9.4 & $\cdots$ & $\ldots$ & $\cdots$ & $\ldots$ & $\ldots$ & $\ldots$ \\
\hline Broad money & 3.4 & 9.1 & 35.3 & 16.4 & $\cdots$ & $\ldots$ & $\cdots$ & $\ldots$ & $\cdots$ & $\ldots$ \\
\hline Velocity (GNP/average broad money) & 4.4 & 4.3 & 4.2 & 3.9 & $\ldots$ & $\ldots$ & $\ldots$ & $\ldots$ & $\ldots$ & $\ldots$ \\
\hline Interest rate $5 /$ & 8.5 & 7.2 & 6.9 & 7.8 & $\ldots$ & $\ldots$ & $\cdots$ & $\cdots$ & $\cdots$ & $\cdots$ \\
\hline & \multicolumn{10}{|c|}{ (In percent of GDP; unless otherwise indicated) } \\
\hline \multicolumn{11}{|l|}{ Investment and saving } \\
\hline Investment & 24.8 & 24.7 & 24.3 & 24.3 & 28.2 & 29.0 & 31.0 & 32.8 & 32.6 & 31.2 \\
\hline Public & 7.5 & 7.8 & 7.1 & 9.8 & 10.8 & 13.1 & 14.1 & 14.9 & 14.6 & 13.1 \\
\hline Private & 11.2 & 15.5 & 17.2 & 14.5 & 17.4 & 15.9 & 16.9 & 17.9 & 18.0 & 18.1 \\
\hline Lesotho Highlands Water Project & 6.0 & 1.5 & 0.0 & 0.0 & 0.0 & 0.0 & 0.0 & 0.0 & 0.0 & 0.0 \\
\hline Gross national savings (including remittances) & 19.1 & 17.2 & 28.6 & 37.0 & 24.4 & 20.5 & 24.4 & 27.2 & 27.3 & 26.2 \\
\hline Public & 13.4 & 12.7 & 19.7 & 26.2 & 19.6 & 16.4 & 17.9 & 20.6 & 20.4 & 18.9 \\
\hline Private & 5.6 & 4.5 & 8.9 & 10.8 & 4.9 & 4.1 & 6.6 & 6.6 & 6.8 & 7.4 \\
\hline \multicolumn{11}{|l|}{ Government budget } \\
\hline Revenue & 47.8 & 50.4 & 57.6 & 63.5 & 60.7 & 54.0 & 52.5 & 53.9 & 54.1 & 54.2 \\
\hline Total grants & 2.7 & 2.2 & 1.1 & 1.3 & 2.0 & 3.7 & 4.8 & 5.8 & 4.9 & 2.9 \\
\hline Total expenditure and net lending & 44.7 & 47.6 & 45.9 & 48.3 & 53.9 & 54.4 & 53.5 & 54.0 & 53.1 & 51.3 \\
\hline Overall balance (excluding grants) & 3.1 & 2.8 & 11.7 & 15.2 & 6.8 & -0.3 & -1.0 & -0.1 & 1.0 & 2.9 \\
\hline Overall balance (including grants) & 5.8 & 5.0 & 12.7 & 16.5 & 8.9 & 3.4 & 3.9 & 5.7 & 5.9 & 5.8 \\
\hline Government debt & 57.4 & 54.5 & 50.3 & 42.9 & 52.4 & 43.1 & 39.6 & 37.3 & 36.1 & 35.5 \\
\hline Domestic debt & 9.1 & 9.3 & 8.1 & 6.7 & 5.6 & 4.8 & 4.3 & 4.1 & 3.9 & 3.8 \\
\hline External debt & 48.3 & 45.2 & 42.1 & 36.2 & 46.7 & 38.3 & 35.3 & 33.2 & 32.1 & 31.6 \\
\hline External debt-service ratio 6/ & 8.2 & 10.0 & 6.4 & 5.0 & 2.8 & 4.5 & 4.5 & 4.9 & 4.8 & 5.0 \\
\hline \multicolumn{11}{|l|}{ Balance of payment } \\
\hline Current account balance (excl. official transfers) & -24.2 & -28.6 & -20.2 & -24.8 & -37.5 & -36.9 & -34.3 & -34.8 & -34.4 & -33.8 \\
\hline Current account balance (incl. official transfers) & -5.7 & -7.5 & 4.3 & 12.7 & -3.7 & -8.5 & -6.5 & -5.6 & -5.3 & -4.9 \\
\hline \multicolumn{11}{|l|}{ Gross official reserves (end of period) } \\
\hline Millions of U.S. dollars & 458 & 501 & 693 & 958 & 982 & 885 & 848 & 861 & 876 & 882 \\
\hline Months of imports of goods and services & 4.0 & 4.3 & 5.7 & 6.7 & 6.6 & 6.3 & 5.9 & 5.7 & 5.6 & 5.5 \\
\hline
\end{tabular}

Sources: Lesotho authorities; and IMF staff estimates and projections.

1/ Fiscal year beginning in April. All fiscal data are reported on a calendar basis

2/ U.S. dollars.

3/ Based on partner-country data, new trade weights from 2004; a minus sign indicates a depreciation.

4 / Change in percent of broad money at the beginning of the period.

$5 /$ The average effective rate on three-month treasury bills. 
Table 2. Lesotho: Central Government Operations, 2005/06-2013/14 1/

(In millions of maloti)

\begin{tabular}{|c|c|c|c|c|c|c|c|c|c|c|c|}
\hline & & & Budget & Prel. Act. & Budget & \multicolumn{6}{|c|}{ Projection } \\
\hline & $2005 / 06$ & $2006 / 07$ & $2007 / 08$ & $2007 / 08$ & $2008 / 09$ & $2008 / 09$ & $2009 / 10$ & $2010 / 11$ & $2011 / 12$ & $2012 / 13$ & $\overline{2013 / 14}$ \\
\hline Revenue & 4,496 & 6,387 & 6,392 & 7,047 & 8,002 & 8,517 & 7,898 & 9,276 & 10,385 & 11,335 & 12,261 \\
\hline Tax revenue & 4,006 & 5,787 & 5,817 & 6,331 & 7,268 & 7,790 & 7,094 & 8,384 & 9,395 & 10,252 & 11,086 \\
\hline Customs revenue (SACU) 2/ & 2,306 & 3,945 & 3,836 & 4,098 & 5,082 & 5,290 & 4,299 & 5,229 & 5,928 & 6,471 & 6,998 \\
\hline Noncustoms tax revenue & 1,700 & 1,842 & 1,981 & 2,233 & 2,191 & 2,499 & 2,795 & 3,155 & 3,467 & 3,782 & 4,087 \\
\hline Income taxes & 925 & 973 & 1,043 & 1,221 & 1,051 & 1,363 & 1,525 & 1,725 & 1,897 & 2,071 & 2,240 \\
\hline Sales tax / value-added tax (VAT) & 656 & 715 & 805 & 848 & 961 & 1,012 & 1,138 & 1,274 & 1,395 & 1,518 & 1,637 \\
\hline Petrol levy & 83 & 66 & 84 & 118 & 90 & 72 & 81 & 91 & 100 & 109 & 118 \\
\hline Other tax revenues & 37 & 86 & 46 & 43 & 85 & 49 & 51 & 65 & 74 & 83 & 93 \\
\hline Nontax revenue & 490 & 600 & 575 & 716 & 733 & 727 & 804 & 892 & 990 & 1,083 & 1,175 \\
\hline Water royalties & 236 & 287 & 272 & 292 & 290 & 341 & 390 & 441 & 489 & 537 & 586 \\
\hline Interest received & 8 & 5 & 7 & 7 & 8 & 6 & 6 & 6 & 6 & 6 & 6 \\
\hline Other nontax revenues & 246 & 308 & 296 & 371 & 443 & 380 & 408 & 445 & 495 & 540 & 584 \\
\hline Grants & 171 & 92 & 500 & 178 & 691 & 306 & 632 & 887 & 1,157 & 964 & 648 \\
\hline Of which: MCC & $\ldots$ & $\ldots$ & $\ldots$ & $\ldots$ & 109 & 213 & 502 & 708 & 924 & 712 & 375 \\
\hline Total expenditure and net lending & 4,291 & 4,860 & 7,225 & 5,965 & 9,096 & 7,657 & 8,248 & 9,379 & 10,379 & 11,069 & 11,597 \\
\hline Current expenditure & 3,604 & 4,136 & 5,311 & 4,687 & 6,957 & 6,169 & 6,156 & 6,901 & 7,483 & 8,057 & 8,635 \\
\hline Wages and salaries & 1,282 & 1,433 & 1,746 & 1,632 & 2,133 & 1,894 & 2,088 & 2,343 & 2,558 & 2,771 & 2,997 \\
\hline Interest payments & 225 & 97 & 367 & 293 & 147 & 109 & 117 & 121 & 129 & 132 & 138 \\
\hline External & 179 & 55 & 304 & 243 & 56 & 46 & 66 & 77 & 86 & 94 & 104 \\
\hline Domestic & 46 & 41 & 62 & 50 & 91 & 63 & 50 & 44 & 42 & 38 & 34 \\
\hline Other expenditure & 2,097 & 2,606 & 3,199 & 2,762 & 4,677 & 4,166 & 3,952 & 4,436 & 4,796 & 5,154 & 5,500 \\
\hline Goods and services & 1,119 & 1,436 & 1,724 & 1,399 & 2,288 & 1,888 & 2,114 & 2,390 & 2,629 & 2,869 & 3,103 \\
\hline Transfer and subsidies & 978 & 1,169 & 1,475 & 1,363 & 2,389 & 2,279 & 1,838 & 2,047 & 2,167 & 2,284 & 2,396 \\
\hline Pensions and gratuities & 296 & 308 & 422 & 396 & 1,160 & 1,113 & 543 & 578 & 610 & 642 & 673 \\
\hline Subventions and transfers & 682 & 862 & 1,053 & 967 & 1,229 & 1,166 & 1,295 & 1,468 & 1,557 & 1,643 & 1,723 \\
\hline Capital expenditure & 697 & 733 & 1,924 & 1,291 & 2,157 & 1,498 & 2,101 & 2,488 & 2,906 & 3,022 & 2,972 \\
\hline Domestically funded & 411.7 & 484.1 & $1,103.5$ & 905.0 & $1,111.8$ & 995.1 & $1,128.8$ & $1,293.9$ & $1,442.3$ & $1,595.2$ & $1,757.1$ \\
\hline Externally funded & 286 & 249 & 821 & 386 & 1,045 & 503 & 973 & 1,194 & 1,464 & 1,426 & 1,215 \\
\hline Grant funded & 171 & 92 & 500 & 178 & 691 & 306 & 632 & 887 & 1,157 & 964 & 648 \\
\hline Of which: MCC & $\ldots$ & $\ldots$ & $\ldots$ & $\ldots$ & 109 & 213 & 502 & 708 & 924 & 712 & 375 \\
\hline Loan funded & 114 & 157 & 321 & 208 & 355 & 197 & 340 & 307 & 307 & 462 & 567 \\
\hline Net lending & -10 & -9 & -10 & -13 & -18 & -10 & -10 & -10 & -10 & -10 & -10 \\
\hline Overall balance, before grants & 204 & 1,527 & -833 & 1,082 & $-1,094$ & 860 & -350 & -103 & 6 & 267 & 664 \\
\hline Overall balance, after grants & 376 & 1,620 & -334 & 1,261 & -404 & 1,166 & 282 & 784 & 1,162 & 1,231 & 1,312 \\
\hline Non-SACU balance $3 /$ & $-1,930$ & $-2,325$ & $-4,170$ & $-2,837$ & $-5,486$ & $-4,125$ & $-4,017$ & $-4,445$ & $-4,766$ & $-5,240$ & $-5,687$ \\
\hline Domestic balance 4/ & 659 & 1,822 & 282 & 1,698 & -11 & 1,399 & 679 & 1,158 & 1,546 & 1,777 & 1,973 \\
\hline Total financing & -376 & $-1,517$ & 334 & $-1,016$ & 404 & $-1,166$ & -282 & -784 & $-1,162$ & $-1,231$ & $-1,312$ \\
\hline External financing & -277 & -266 & 52 & 22 & 135 & 42 & 123 & 77 & 60 & 213 & 308 \\
\hline Loan drawings & 114 & 157 & 321 & 208 & 355 & 197 & 340 & 307 & 307 & 462 & 567 \\
\hline Amortization & -391 & -423 & -269 & -186 & -220 & -155 & -217 & -230 & -247 & -249 & -260 \\
\hline Domestic financing & -99 & $-1,251$ & 282 & $-1,038$ & 269 & $-1,208$ & -405 & -861 & $-1,222$ & $-1,444$ & $-1,619$ \\
\hline Bank & -102 & $-1,248$ & $\ldots$ & -999 & $\ldots$ & $\ldots$ & -291 & $\ldots$ & $\ldots$ & $\ldots$ & $\ldots$ \\
\hline Nonbank & 3 & -3 & $\ldots$ & -39 & $\ldots$ & $\ldots$ & -115 & $\ldots$ & $\ldots$ & $\ldots$ & $\ldots$ \\
\hline Statistical discrepancy & 0 & 102 & 0 & 245 & 0 & 0 & 0 & 0 & 0 & 0 & 0 \\
\hline
\end{tabular}


Table 2. Lesotho: Central Government Operations, 2005/06-2013/14 1/ (concluded)

(In percent of GDP; unless otherwise indicated)

\begin{tabular}{|c|c|c|c|c|c|c|c|c|c|c|c|}
\hline & \multirow{2}{*}{$\begin{array}{r}\text { Act } \\
2005 / 06 \\
\end{array}$} & \multirow{2}{*}{$\begin{array}{r}\text { Act. } \\
2006 / 07 \\
\end{array}$} & \multirow{2}{*}{$\begin{array}{r}\text { Budget } \\
2007 / 08 \\
\end{array}$} & \multirow{2}{*}{$\begin{array}{r}\text { Prel. Act. } \\
2007 / 08 \\
\end{array}$} & \multirow{2}{*}{$\begin{array}{r}\text { Budget } \\
2008 / 09 \\
\end{array}$} & \multicolumn{6}{|c|}{ Projection } \\
\hline & & & & & & $2008 / 09$ & $2009 / 10$ & $2010 / 11$ & $2011 / 12$ & $2012 / 13$ & $2013 / 14$ \\
\hline Revenue & 49.2 & 60.0 & 53.0 & 57.8 & 59.3 & 61.8 & 51.2 & 53.2 & 54.1 & 54.1 & 54.1 \\
\hline Customs revenue (SACU) $2 /$ & 25.3 & 37.1 & 31.8 & 33.6 & 37.6 & 38.4 & 27.9 & 30.0 & 30.9 & 30.9 & 30.9 \\
\hline Noncustoms tax revenue & 18.6 & 17.3 & 16.4 & 18.3 & 16.2 & 18.1 & 18.1 & 18.1 & 18.1 & 18.1 & 18.0 \\
\hline Income taxes & 10.1 & 9.1 & 8.7 & 10.0 & 7.8 & 9.9 & 9.9 & 9.9 & 9.9 & 9.9 & 9.9 \\
\hline Sales tax / value-added tax (VAT) & 7.2 & 6.7 & 6.7 & 7.0 & 7.1 & 7.3 & 7.4 & 7.3 & 7.3 & 7.3 & 7.2 \\
\hline Petrol levy & 0.9 & 0.6 & 0.7 & 1.0 & 0.7 & 0.5 & 0.5 & 0.5 & 0.5 & 0.5 & 0.5 \\
\hline Other tax revenues & 0.4 & 0.8 & 0.4 & 0.4 & 0.6 & 0.4 & 0.3 & 0.4 & 0.4 & 0.4 & 0.4 \\
\hline Nontax revenue & 5.4 & 5.6 & 4.8 & 5.9 & 5.4 & 5.3 & 5.2 & 5.1 & 5.2 & 5.2 & 5.2 \\
\hline Water royalties & 2.6 & 2.7 & 2.3 & 2.4 & 2.1 & 2.5 & 2.5 & 2.5 & 2.5 & 2.6 & 2.6 \\
\hline Interest received & 0.1 & 0.0 & 0.1 & 0.1 & 0.1 & 0.0 & 0.0 & 0.0 & 0.0 & 0.0 & 0.0 \\
\hline Other nontax revenues & 2.7 & 2.9 & 2.5 & 3.0 & 3.3 & 2.8 & 2.6 & 2.5 & 2.6 & 2.6 & 2.6 \\
\hline Grants & 1.9 & 0.9 & 4.1 & 1.5 & 5.1 & 2.2 & 4.1 & 5.1 & 6.0 & 4.6 & 2.9 \\
\hline Of which: MCC & $\ldots$ & $\ldots$ & $\ldots$ & $\ldots$ & 0.8 & 1.5 & 3.3 & 4.1 & 4.8 & 3.4 & 1.7 \\
\hline Total expenditure and net lending & 47.0 & 45.6 & 59.9 & 48.9 & 67.4 & 55.5 & 53.5 & 53.8 & 54.1 & 52.9 & 51.2 \\
\hline Current expenditure & 39.5 & 38.8 & 44.1 & 38.5 & 51.5 & 44.7 & 39.9 & 39.6 & 39.0 & 38.5 & 38.1 \\
\hline Wages and salaries & 14.0 & 13.5 & 14.5 & 13.4 & 15.8 & 13.7 & 13.5 & 13.4 & 13.3 & 13.2 & 13.2 \\
\hline Interest payments & 2.5 & 0.9 & 3.0 & 2.4 & 1.1 & 0.8 & 0.8 & 0.7 & 0.7 & 0.6 & 0.6 \\
\hline External & 2.0 & 0.5 & 2.5 & 2.0 & 0.4 & 0.3 & 0.4 & 0.4 & 0.5 & 0.4 & 0.5 \\
\hline Domestic & 0.5 & 0.4 & 0.5 & 0.4 & 0.7 & 0.5 & 0.3 & 0.3 & 0.2 & 0.2 & 0.2 \\
\hline Other expenditure & 23.0 & 24.5 & 26.5 & 22.7 & 34.6 & 30.2 & 25.6 & 25.4 & 25.0 & 24.6 & 24.3 \\
\hline Goods and services & 12.3 & 13.5 & 14.3 & 11.5 & 16.9 & 13.7 & 13.7 & 13.7 & 13.7 & 13.7 & 13.7 \\
\hline Transfers and subsidies & 10.7 & 11.0 & 12.2 & 11.2 & 17.7 & 16.5 & 11.9 & 11.7 & 11.3 & 10.9 & 10.6 \\
\hline Capital expenditure & 7.6 & 6.9 & 16.0 & 10.6 & 16.0 & 10.9 & 13.6 & 14.3 & 15.1 & 14.4 & 13.1 \\
\hline Of which: domestically funded & 4.5 & 4.5 & 9.2 & 7.4 & 8.2 & 7.2 & 7.3 & 7.4 & 7.5 & 7.6 & 7.8 \\
\hline Net lending & -0.1 & -0.1 & -0.1 & -0.1 & -0.1 & -0.1 & -0.1 & -0.1 & -0.1 & 0.0 & 0.0 \\
\hline Overall balance, before grants & 2.2 & 14.3 & -6.9 & 8.9 & -8.1 & 6.2 & -2.3 & -0.6 & 0.0 & 1.3 & 2.9 \\
\hline Overall balance, after grants & 4.1 & 15.2 & -2.8 & 10.3 & -3.0 & 8.5 & 1.8 & 4.5 & 6.1 & 5.9 & 5.8 \\
\hline Non-SACU balance 3/ & -21.1 & -21.8 & -34.6 & -23.3 & -40.6 & -29.9 & -26.0 & -25.5 & -24.8 & -25.0 & -25.1 \\
\hline Domestic balance 4/ & 7.2 & 17.1 & 2.3 & 13.9 & -0.1 & 10.1 & 4.4 & 6.6 & 8.1 & 8.5 & 8.7 \\
\hline Total financing & -4.1 & -14.3 & 2.8 & -8.3 & 3.0 & -8.5 & -1.8 & -4.5 & -6.1 & -5.9 & -5.8 \\
\hline External financing & -3.0 & -2.5 & 0.4 & 0.2 & 1.0 & 0.3 & 0.8 & 0.4 & 0.3 & 1.0 & 1.4 \\
\hline Loan drawings & 1.3 & 1.5 & 2.7 & 1.7 & 2.6 & 1.4 & 2.2 & 1.8 & 1.6 & 2.2 & 2.5 \\
\hline Amortization & -4.3 & -4.0 & -2.2 & -1.5 & -1.6 & -1.1 & -1.4 & -1.3 & -1.3 & -1.2 & -1.1 \\
\hline Domestic financing & -1.1 & -11.8 & 2.3 & -8.5 & 2.0 & -8.8 & -2.6 & -4.9 & -6.4 & -6.9 & -7.1 \\
\hline Bank & -1.1 & -11.7 & 0.0 & -8.2 & 0.0 & 0.0 & -1.9 & $\ldots$ & $\ldots$ & $\ldots$ & $\ldots$ \\
\hline Nonbank & 0.0 & 0.0 & 0.0 & -0.3 & 0.0 & 0.0 & -0.7 & $\ldots$ & $\ldots$ & $\ldots$ & $\ldots$ \\
\hline Statistical discrepancy & 0 & 1.0 & 0.0 & 2.0 & 0 & 0 & 0 & 0 & 0 & 0 & 0 \\
\hline \multicolumn{12}{|l|}{ Memorandum items: } \\
\hline Non-SACU balance & -21.1 & -21.8 & -34.6 & -23.3 & -40.6 & -29.9 & -26.0 & -25.5 & -24.8 & -25.0 & -25.1 \\
\hline GNP at current prices (Millions of maloti) $5 /$ & 10,683 & 12,844 & 13,961 & 14,722 & 16,994 & 16,898 & 17,998 & 20,298 & 22,224 & 24,244 & 26,178 \\
\hline GDP at current prices (Millions of maloti) 5/ & 9130.1 & 10646.43 & 12,052 & 12,188 & 13,501 & 13,788 & 15,427 & 17,445 & 19,187 & 20,943 & 22,651 \\
\hline
\end{tabular}

Sources: Ministry of Finance, and Fund staff estimates and projections.

1/ Fiscal year from April to March.

2/ Adjustment receipts of M 330 million in 2005/06 included.

$3 /$ Overall balance excluding customs revenue (SACU)

4/ Domestic balance excludes grants, foreign-financed capital spending, foreign interest payments, and exceptional factors.

$5 /$ On a fiscal year basis. 
Table 3. Lesotho: Balance of Payments, 2005-2013

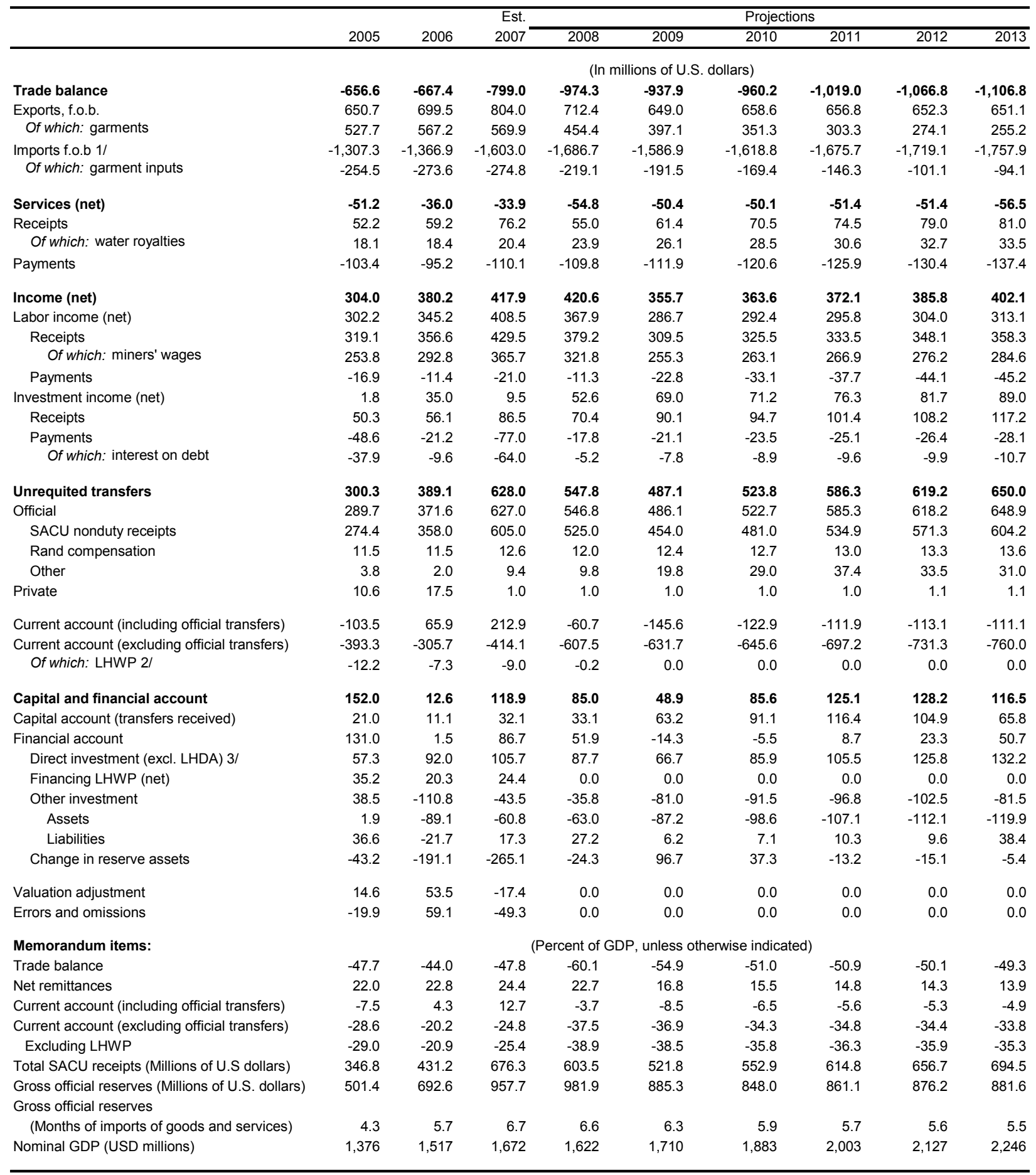

Sources: Central Bank of Lesotho; and IMF staff estimates and projections.

1/ Adjusted for SACU duty receipts.

2/ Lesotho Highlands Water Project.

3/ Lesotho Highlands Development Authority. 
Table 4. Lesotho: Monetary Survey, 2003-08

\begin{tabular}{|c|c|c|c|c|c|c|c|}
\hline & \multirow[b]{2}{*}{2002} & \multicolumn{5}{|c|}{ December } & \multirow{2}{*}{$\begin{array}{r}\text { September } \\
2008\end{array}$} \\
\hline & & 2003 & 2004 & 2005 & 2006 & 2007 & \\
\hline & \multicolumn{7}{|c|}{ (In millions of maloti; unless otherwise indicated) } \\
\hline Net foreign assets & $3,891.1$ & $3,496.3$ & $4,012.0$ & $4,306.9$ & $6,205.3$ & $8,520.6$ & $10,015.8$ \\
\hline Central bank & $3,321.3$ & $2,873.1$ & $2,886.4$ & $3,151.7$ & $4,373.6$ & $6,289.9$ & $7,815.6$ \\
\hline Commercial banks & 569.8 & 623.2 & $1,125.6$ & $1,155.2$ & $1,831.7$ & $2,230.7$ & $2,200.2$ \\
\hline Net domestic assets & $-1,722.9$ & $-1,198.4$ & $-1,636.4$ & $-1,714.3$ & $-2,697.4$ & $-4,439.1$ & $-5,634.7$ \\
\hline Domestic credit & 747.2 & 340.5 & -146.8 & -100.5 & -576.8 & $-2,071.1$ & $-2,405.3$ \\
\hline Claims on central government (net) & -317.5 & -167.0 & -712.1 & -910.1 & $-1,498.8$ & $-3,324.3$ & $-3,939.2$ \\
\hline Central bank & $-1,066.0$ & $-1,090.1$ & $-1,197.8$ & $-1,199.9$ & $-1,973.9$ & $-3,728.8$ & $-4,318.5$ \\
\hline Commercial banks & 748.5 & 923.1 & 485.7 & 289.8 & 475.2 & 404.5 & 379.3 \\
\hline Claims on the rest of the economy & $1,064.7$ & 507.5 & 565.2 & 809.6 & 921.9 & $1,253.3$ & $1,533.9$ \\
\hline Other items (net) & $-2,470.1$ & $-1,538.9$ & $-1,489.6$ & $-1,613.9$ & $-2,120.6$ & $-2,368.1$ & $-3,229.4$ \\
\hline Money and quasi-money (M2) & $2,168.2$ & $2,297.9$ & $2,375.5$ & $2,592.6$ & $3,507.9$ & $4,081.5$ & $4,381.1$ \\
\hline Money & $1,440.9$ & $1,537.7$ & $1,591.9$ & $1,832.0$ & $2,688.8$ & $2,990.7$ & $3,350.3$ \\
\hline \multicolumn{8}{|l|}{ Of which: } \\
\hline Currency outside dep. mon. banks & 179.7 & 183.5 & 204.5 & 212.8 & 309.4 & 339.3 & 379.6 \\
\hline Demand deposits & $1,261.3$ & $1,354.2$ & $1,387.4$ & $1,619.3$ & $2,379.4$ & $2,651.4$ & $2,970.7$ \\
\hline Quasimoney & 727.2 & 760.1 & 783.6 & 760.5 & 819.1 & $1,090.8$ & $1,030.7$ \\
\hline \multicolumn{8}{|l|}{ Of which: } \\
\hline \multirow[t]{2}{*}{ Time and savings deposits } & 727.2 & 760.1 & 783.6 & 760.5 & 819.1 & $1,090.8$ & $1,030.7$ \\
\hline & \multicolumn{7}{|c|}{ (Annual percent changes) } \\
\hline Net foreign assets $1 /$ & -66.4 & -18.2 & 22.4 & 12.4 & 73.2 & 66.0 & 52.8 \\
\hline Central bank & -60.0 & -20.7 & 0.6 & 11.2 & 47.1 & 54.6 & 44.6 \\
\hline Commercial banks & -6.4 & 2.5 & 21.9 & 1.2 & 26.1 & 11.4 & 8.2 \\
\hline Net domestic assets $1 /$ & 75.2 & 24.2 & -19.1 & -3.3 & -37.9 & -49.7 & -36.5 \\
\hline Claims on central government (net) & 15.6 & 6.9 & -23.7 & -8.3 & -22.7 & -52.0 & -22.3 \\
\hline Claims on the rest of the economy & 6.6 & -25.7 & 2.5 & 10.3 & 4.3 & 9.4 & 9.1 \\
\hline Claims on the rest of the econ. (yearly change) & 14.1 & -52.3 & 11.4 & 43.2 & 13.9 & 35.9 & 28.8 \\
\hline Other items (net) & 53.0 & 42.9 & 2.1 & -5.2 & -19.5 & -7.1 & -23.3 \\
\hline Money and quasimoney (M2) 1/ & 8.8 & 6.0 & 3.4 & 9.1 & 35.3 & 16.4 & 16.2 \\
\hline
\end{tabular}

Sources: Central Bank of Lesotho; and IMF staff estimates and projections.

1/ Annual change in percent of beginning-of-year M2, unless otherwise indicated. September is year-on-year. 
Table 5. Lesotho: Indicators of External Vulnerability, 2003-07

(In percent of GDP; unless otherwise indicated)

\begin{tabular}{|c|c|c|c|c|c|}
\hline & 2003 & 2004 & 2005 & 2006 & 2007 \\
\hline \multicolumn{6}{|l|}{ Financial indicators - testing } \\
\hline Public sector debt $1 /$ & 76.2 & 57.4 & 54.5 & 50.3 & 49.1 \\
\hline Broad money (M2; annual percent change) & 6.0 & 3.4 & 9.1 & 35.3 & 16.4 \\
\hline Private sector credit (annual percent change) & -25.7 & 2.5 & 10.3 & 4.3 & 9.4 \\
\hline Treasury-bill yield (percent) 2/ & 12.0 & 8.5 & 7.2 & 6.9 & 7.8 \\
\hline Treasury-bill yield (real, percent) $3 /$ & 4.7 & 3.5 & 3.8 & 0.8 & -0.2 \\
\hline \multicolumn{6}{|l|}{ External indicators } \\
\hline Exports of goods and services (U.S. dollars, annual percent change) & 33.4 & 48.9 & -9.2 & 7.9 & 16.0 \\
\hline Imports of goods and services (U.S. dollars, annual percent change) & 31.9 & 29.7 & 1.5 & 3.6 & 17.2 \\
\hline Current account balance & -12.8 & -5.7 & -7.5 & 4.3 & 12.7 \\
\hline Capital and financial account balance & 12.3 & 7.0 & 11.0 & 0.8 & 7.1 \\
\hline Of which: inward foreign direct investment & 4.2 & 4.1 & 4.2 & 6.1 & 6.3 \\
\hline Net foreign assests of the banking sector (millions of U.S. dollars) & 93.8 & 199.9 & 182.6 & 262.8 & 327.6 \\
\hline Foreign assets of the banking sector (millions of U.S. dollars) & 128.4 & 224.1 & 199.5 & 273.3 & 338.3 \\
\hline Foreign liabilities of the banking sector (millions of U.S. dollars) & 34.6 & 24.1 & 16.9 & 10.5 & 10.8 \\
\hline Gross official reserves (millions of U.S. dollars) & 446.5 & 458.2 & 501.4 & 692.6 & 957.7 \\
\hline Gross official reserves (months of imports of goods and services) & 5.0 & 4.0 & 4.3 & 5.7 & 6.7 \\
\hline Ratio of reserve money to reserves (percent) & 17.5 & 21.7 & 23.1 & 14.1 & 10.5 \\
\hline Ratio of broad money to reserves (percent) & 77.5 & 92.1 & 81.7 & 72.7 & 62.6 \\
\hline Total external debt $4 /$ & 69.5 & 55.4 & 45.5 & 40.9 & 37.4 \\
\hline Ratio of total external debt to exports of goods and services (percent) & 133.0 & 92.3 & 89.0 & 81.8 & 71.1 \\
\hline \multicolumn{6}{|l|}{ Memorandum items: } \\
\hline GDP (millions of U.S. dollars) & 994 & 1,290 & 1,376 & 1,517 & 1,672 \\
\hline Nominal exchange rate (maloti per U.S. dollar, end-of-period) & 6.6 & 5.6 & 6.3 & 7.0 & 6.8 \\
\hline
\end{tabular}

Sources: Lesotho authorities; and IMF staff estimates and projections.

1/ National government debt.

2/ End of period.

3/ Backward-looking with actual consumer price index (CPI).

4/ Excludes private debt within the Common Monetary Area. 
Table 6. Lesotho: Millennium Development Goals

\begin{tabular}{|c|c|c|c|c|}
\hline & 1990 & 1995 & 2000 & 2007 \\
\hline \multicolumn{5}{|l|}{ Goal 1: Eradicate extreme poverty and hunger } \\
\hline Employment to population ratio, $15+$, total (in percent) & 54.0 & 54.0 & 35.0 & 37.0 \\
\hline Employment to population ratio, ages $15-24$, total (in percent) & 40.0 & 39.0 & 24.0 & 25.0 \\
\hline Income share held by lowest 20 percent & $\ldots$ & 1.5 & $\ldots$ & $\ldots$ \\
\hline Malnutrition prevalence, weight for age (percent of children under 5) & $\ldots$ & $\ldots$ & 15.0 & 16.6 \\
\hline Poverty headcount ratio at national poverty line (percent of population) & $\ldots$ & 49.2 & 68.0 & $\ldots$ \\
\hline Prevalence of undernourishment (percent of population) & 17.0 & 14.0 & $\ldots$ & $\ldots$ \\
\hline Vulnerable employment, total (percent of total employment) & $\ldots$ & $\ldots$ & $\ldots$ & $\ldots$ \\
\hline \multicolumn{5}{|l|}{ Goal 2: Achieve universal primary education } \\
\hline Literacy rate, youth female (percent of females ages 15-24) & $\ldots$ & $\ldots$ & $\ldots$ & $\ldots$ \\
\hline Literacy rate, youth male (percent of males ages $15-24$ ) & $\ldots$ & $\ldots$ & $\ldots$ & $\ldots$ \\
\hline Persistence to last grade of primary, total (percent of cohort) & $\ldots$ & $\ldots$ & 54.0 & 62.0 \\
\hline Primary completion rate, total (percent of relevant age group) & 56.0 & 63.0 & 60.0 & 78.0 \\
\hline Total enrollment, primary (percent net) & $\ldots$ & $\ldots$ & 78.0 & 75.0 \\
\hline Goal 3: Promote gender equality and empower women & 132.0 & & 117.5 & 113.5 \\
\hline Proportion of seats held by women in national parliament (in percent) & $\ldots$ & 5.0 & 4.0 & 24.0 \\
\hline Ratio of female to male enrollments in tertiary education & 130.0 & $\ldots$ & 153.0 & 119.0 \\
\hline Ratio of female to male primary enrollment & 122.0 & $\ldots$ & 104.0 & 100.0 \\
\hline Ratio of female to male secondary enrollment & 142.0 & $\ldots$ & 131.0 & 127.0 \\
\hline Ratio of young literate females to males (percent ages 15-24) & $\ldots$ & $\ldots$ & $\ldots$ & $\ldots$ \\
\hline \multicolumn{5}{|l|}{ Share of women employed in the nonagricultural sector } \\
\hline (in percent of total nonagricultural employment) & $\ldots$ & 44.1 & $\ldots$ & $\ldots$ \\
\hline \multicolumn{5}{|l|}{ Goal 4: Reduce child mortality } \\
\hline Immunization, measles (percent of children ages $12-23$ months) & 80.0 & 83.0 & 74.0 & 85.0 \\
\hline Mortality rate, infant (per 1,000 live births) & 81.0 & 73.0 & 86.0 & 102.0 \\
\hline Mortality rate, under-5 (per 1,000$)$ & 101.0 & 91.0 & 108.0 & 132.0 \\
\hline \multicolumn{5}{|l|}{ Goal 5: Improve maternal health } \\
\hline Adolescent fertility rate (births per 1,000 women ages $15-19$ ) & $\ldots$ & 94.0 & 91.0 & 77.0 \\
\hline Births attended by skilled health staff (percent of total) & $\ldots$ & 50.0 & 60.0 & $\ldots$ \\
\hline Contraceptive prevalence (percent of women ages 15-49) & 23.0 & $\ldots$ & 30.0 & $\ldots$ \\
\hline Maternal mortality ratio (modeled estimate, per 100,000 live births) & $\ldots$ & $\ldots$ & $\ldots$ & 960.0 \\
\hline Pregnant women receiving prenatal care (in percent) & $\ldots$ & 88.0 & 85.0 & $\ldots$ \\
\hline Unmet need for contraception (percent of married women ages 15-49) & $\ldots$ & $\ldots$ & $\ldots$ & $\ldots$ \\
\hline \multicolumn{5}{|l|}{ Goal 6: Combat HIVIAIDS, malaria, and other diseases } \\
\hline Children with fever receiving antimalarial drugs (percent of children under age 5 with fever) & $\ldots$ & $\ldots$ & $\ldots$ & $\ldots$ \\
\hline Condom use, population ages $15-24$, female (percent of females ages $15-24$ ) & $\ldots$ & $\ldots$ & $\ldots$ & $\ldots$ \\
\hline Condom use, population ages $15-24$, male (percent of males ages $15-24$ ) & $\ldots$ & $\ldots$ & $\ldots$ & $\ldots$ \\
\hline Incidence of tuberculosis (per 100,000 people) & 184.0 & 323.0 & 553.0 & 635.0 \\
\hline Prevalence of HIV, female (percent ages 15-24) & $\ldots$ & $\ldots$ & $\ldots$ & 14.9 \\
\hline Prevalence of HIV, total (percent of population ages 15-49) & $\ldots$ & $\ldots$ & 23.9 & 23.2 \\
\hline Tuberculosis cases detected under DOTS (in percent) & $\ldots$ & 59.0 & 72.0 & 79.0 \\
\hline \multicolumn{5}{|l|}{ Goal 7: Ensure environmental sustainability } \\
\hline Annual freshwater withdrawals, total (percent of internal resources) & $\ldots$ & $\ldots$ & 1.0 & $\ldots$ \\
\hline CO2 emissions (kg per PPP \$ of GDP) & $\ldots$ & $\ldots$ & $\ldots$ & $\ldots$ \\
\hline $\mathrm{CO} 2$ emissions (metric tons per capita) & $\ldots$ & $\ldots$ & $\ldots$ & $\ldots$ \\
\hline Forest area (percent of land area) & 0.0 & $\ldots$ & 0.0 & 0.0 \\
\hline Improved sanitation facilities (percent of population with access) & $\ldots$ & 33.0 & 34.0 & 36.0 \\
\hline Improved water source (percent of population with access) & $\ldots$ & 77.0 & 77.0 & 78.0 \\
\hline Marine protected areas, (percent of surface area) & $\ldots$ & $\ldots$ & $\ldots$ & $\ldots$ \\
\hline Nationally protected areas (percent of total land area) & $\ldots$ & $\ldots$ & $\ldots$ & $\ldots$ \\
\hline \multicolumn{5}{|l|}{ Goal 8: Develop a global partnership for development } \\
\hline Aid per capita (current US\$) & 87.0 & 65.0 & 19.0 & 36.0 \\
\hline Debt service (PPG and IMF only, percent of exports of G\&S, excl. workers' remittances) & 4.2 & 6.1 & 11.3 & 4.0 \\
\hline Internet users (per 100 people) & $\ldots$ & 0.0 & 0.2 & 3.5 \\
\hline Mobile phone subscribers (per 100 people) & $\ldots$ & $\ldots$ & 1.1 & 22.7 \\
\hline Telephone mainlines (per 100 people) & 0.8 & 1.0 & 1.2 & 2.7 \\
\hline \multicolumn{5}{|l|}{ Other } \\
\hline Fertility rate, total (births per woman) & 4.9 & 4.5 & 4.0 & 3.5 \\
\hline GNI per capita, Atlas method (current US\$) & 640.0 & 770.0 & 590.0 & $1,000.0$ \\
\hline GNI, Atlas method (current US\$) (billions) & 1.0 & 1.3 & 1.1 & 2.0 \\
\hline Gross capital formation (percent of GDP) & 52.7 & 60.6 & 42.6 & 36.1 \\
\hline Life expectancy at birth, total (years) & 59.0 & 57.0 & 49.0 & 43.0 \\
\hline Literacy rate, adult total (percent of people ages 15 and above) & $\ldots$ & $\ldots$ & 82.0 & $\ldots$ \\
\hline Population, total (in millions) & 1.6 & 1.7 & 1.9 & 2.0 \\
\hline Trade (percent of GDP) & 139.3 & 141.8 & 123.1 & 150.5 \\
\hline
\end{tabular}

Source: World Development Indicators database. 


\section{INTERNATIONAL MONETARY FUND}

\section{KINGDOM OF LESOTHO}

\section{Staff Report for the 2008 Article IV Consultation-Informational Annex}

Prepared by the Staff Representatives for the 2008 Consultation with the Kingdom of Lesotho Approved by Robert Sharer and Anthony Boote

January 23, 2009

- $\quad$ Relations with the Fund. Describes financial and technical assistance by the IMF, and provides information on the exchange rate system. Lesotho's last Fund arrangement, a PRGF, expired in 2004.

- The JMAP Bank-Fund. Describes the priorities and main activities of the World Bank Group and the IMF, and areas of cooperation in their work with the authorities of Lesotho.

- $\quad$ Statistical Issues. Assesses the quality of statistical data. Economic data are adequate for surveillance purposes, though some deficiencies remain. Lesotho subscribes to the GDDS. 


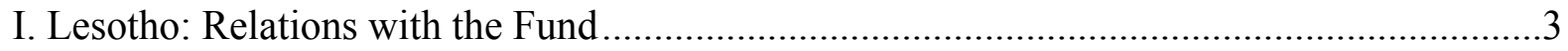

II. Lesotho: The JMAP Bank-Fund Matrix....................................................................6

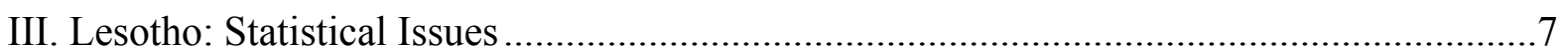




\section{LESOTHO: RELATIONS WITH THE FUND}

I. Membership status: Joined 07/25/1968; accepted the obligations of Article VIII, Sections 2, 3, and 4: 03/05/1997.

II. General resources account:

Quota

Fund holdings of currency

Reserve position in Fund

III. SDR Department:

Net cumulative allocation

Holdings

IV. Outstanding purchases and loans:

PRGF arrangements

\section{$\underline{\text { SDR Million }}$}

34.90

31.32

3.61

$\underline{\text { SDR Million }}$

3.74

3.98

$\underline{\text { SDR Million }}$

20.65 $\underline{\text { Percent Quota }}$

100.00

89.75

10.35

$\underline{\text { Percent Allocation }}$

100.00

106.39

$\underline{\text { Percent Quota }}$

59.17

V. Financial arrangements:

$\begin{array}{ccccc}\text { Type } & \begin{array}{c}\text { Approval } \\ \text { Date }\end{array} & \begin{array}{c}\text { Expiration } \\ \text { Date }\end{array} & \begin{array}{c}\text { Amount Approved } \\ \text { (SDR Million) }\end{array} & \begin{array}{c}\text { Amount Drawn } \\ \text { (SDR Million) }\end{array} \\ \text { PRGF } & 03 / 09 / 2001 & 10 / 31 / 2004 & 24.50 & 24.50 \\ \text { Stand-By } & 09 / 23 / 1996 & 9 / 22 / 1997 & 7.17 & 0.0 \\ \text { Stand-by } & 07 / 31 / 1995 & 7 / 30 / 1996 & 7.17 & 0.0\end{array}$

VI. Projected obligations to fund (SDR million; based on existing use of resources and present holdings of SDRs):

\begin{tabular}{lllll} 
Forthcoming \\
\hline$\underline{2008}$ & $\underline{2009}$ & $\underline{2010}$ & $\underline{2011}$ & $\underline{2012}$
\end{tabular}

$\begin{array}{llllll}\text { Principal } & 1.40 & 3.85 & 4.90 & 4.55 & 3.15 \\ \text { Charges/Interest } & 0.05 & 0.09 & 0.06 & 0.04 & 0.02 \\ \text { Total } & \mathbf{1 . 4 5} & \mathbf{3 . 9 4} & \mathbf{4 . 9 6} & \mathbf{4 . 5 9} & \mathbf{3 . 1 7}\end{array}$


VII. Under the Fund's safeguards assessment policy, the CBL was subject to a safeguards assessment with respect to PRGF arrangement approved on March 09, 2001. The assessment, completed on July 24, 2003, found that while the bank has made notable progress in strengthening its safeguards since the off-site assessment in July 2001, vulnerabilities remain, in particular in the areas of internal audit and internal controls.

\section{Exchange arrangement:}

Lesotho's currency, the loti (plural maloti), is pegged to the South African rand at par. The CBL deals with commercial banks mainly in South African rand. As of December 23, 2008, the rand (maloti) rate per U.S. dollar was M 9.74.

\section{Article IV consultation:}

The 2007 Article IV consultation was concluded by the Executive Board on November 14, 2007. Lesotho is on the standard 12-month Article IV consultation cycle.

\section{Technical assistance missions:}

$\begin{array}{llll}\text { Monetary policy implementation } & \text { MAE } & \text { short-term } & 2001 \\ \text { Public accounts } & \text { FAD } & \text { short-term } & 2002 \\ \text { General Data Dissemination System (GDDS) project for } & \text { STA } & \text { short-term } & 2002 \\ \text { Anglophone Africa } & & & \\ \text { Balance of payments } & \text { STA } & \text { short-term } & 2002 \\ \text { Monetary policy and AML/CFT } & \text { MFD } & \text { short-term } & 2003 \\ \text { Government finance statistics } & \text { STA } & \text { short-term } & 2003 \\ \text { National accounts } & \text { STA } & \text { short-term } & 2003 \\ \text { National accounts statistics } & \text { STA } & \text { short-term } & 2004 \\ \text { GDDS project for Anglophone Africa } & \text { STA } & \text { short-term } & 2004 \\ \text { Legislative drafting/FIU } & \text { LEG } & \text { Short-term } & 2005 \\ \text { Monetary operations/payments issues/banking supervision } & \text { MFD } & \text { short-term } & 2005 \\ \text { Government finance statistics/GDDS } & \text { STA } & \text { short-term } & 2005 \\ \text { Banking law } & \text { LEG } & \text { short-term } & 2005 \\ \text { National accounts statistics/GDDS } & \text { STA } & \text { short-term } & 2005 \\ \text { Monetary operations } & \text { MFD } & \text { short-term } & 2005 \\ \text { Payments issues } & \text { MFD } & \text { short-term } & 2005 \\ \text { Banking law } & \text { LEG } & \text { short-term } & 2005 \\ \text { BOP statistics/GDDS } & \text { STA } & \text { short-term } & 2005 \\ \text { GDDS consultation } & \text { STA } & \text { short-term } & 2005 \\ \text { Government Finance Statistics: GDDS Project for Anglophone } & \text { STA } & \text { short-term } & 2006 \\ \text { African Countries } & & & \\ \text { Payments System Reform } & \text { LEG } & \text { short-term } & 2006 \\ \text { Central Banking and Banking Law } & \text { LEG } & \text { short-term } & 2006 \\ \text { Bank Supervision/Monetary Operations/Payments \& Settlements } & \text { MFD } & \text { short-term } & 2006 \\ \text { Legal drafting, AML/CFT, FIU, supervisory and institutional } & \text { LEG } & \text { short-term } & 2006 \\ \text { Regional workshop on the statistical treatment of SACU transfers } & \text { STA } & \text { short-term } & 2007\end{array}$


Monetary and Financial Statistics

Nonbank supervision

Bank supervision

Monetary operations/Bank supervision

Payments systems

Government Finance Statistics: GDDS Project for Anglophone

African Countries

Monetary operations

Legal drafting of the Financial Institutions Act

Balance of payment

Monetary operations/Bank supervision

Missions planned for 2009

Bank supervision

Money market

National accounts

Pension supervision

$\begin{array}{lll}\text { STA } & \text { short-term } & 2007 \\ \text { MCM } & \text { short-term } & 2007 \\ \text { MCM } & \text { short-term } & 2007 \\ \text { MCM } & \text { short-term } & 2007 \\ \text { MCM } & \text { short-term } & 2007 \\ \text { STA } & \text { short-term } & 2008 \\ & & \\ \text { MCM } & \text { short-term } & 2008 \\ \text { LEG } & \text { short-term } & 2008 \\ \text { STA } & \text { short-term } & 2008 \\ \text { MCM } & \text { short-term } & 2008\end{array}$

MCM short-term 2009

MCM short-term 2009

STA short-term 2009

MCM short-term 2009

\section{Resident Representatives:}

The Senior Resident Representative posted in South Africa has been covering Lesotho since January 2004. 


\section{LESOTHO: ThE JMAP BANK-FUND MATRIX}

(As of December 15, 2008)

\begin{tabular}{|c|c|c|c|}
\hline Title & Products & $\begin{array}{l}\text { Provisional Timing of } \\
\text { missions }\end{array}$ & $\begin{array}{c}\text { Expected } \\
\text { Delivery date }\end{array}$ \\
\hline \multicolumn{4}{|c|}{ A. Mutual information on relevant work programs } \\
\hline $\begin{array}{l}\text { Bank work } \\
\text { program in next } \\
12 \text { months }\end{array}$ & $\begin{array}{l}\text { - CAS } \\
\text { - ESW on Regional Trade and } \\
\text { Integration } \\
\text { - PRSC series of three operations } \\
\text { - Private Sector competitiveness and } \\
\text { Economic Diversification project } \\
\text { - A series of policy notes supporting the } \\
\text { authorities' growth strategy. }\end{array}$ & $\begin{array}{l}\text { - July/August } 2008 \\
\text { - July } 2008 \\
\text { - August/September } 2008 \\
\text { - Ongoing regular } \\
\text { - } \text { missions } \\
\text { - Ongoing }\end{array}$ & $\begin{array}{l}\text { - } 2009 \\
\text { - } \text { May } 2008 \\
\text { for first } \\
\text { release } \\
\text { - } \text { Project } \\
\text { effective }\end{array}$ \\
\hline $\begin{array}{l}\text { IMF work } \\
\text { program in next } \\
12 \text { months }\end{array}$ & $\begin{array}{l}\text { - One-person, one-day staff visit } \\
\text { - } 2008 \text { Article IV Consultation } \\
\text { - IMF Working Paper on SACU growth } \\
\text { performance and macroeconomic } \\
\text { synchronization. }\end{array}$ & $\begin{array}{ll}\text { - } & \text { June-July } 2008 \\
\text { - } & \text { Oct. 29-Nov. 11, } 2008 \\
\text { - } & \text { N/A }\end{array}$ & $\begin{array}{l}\text { - } \mathrm{N} / \mathrm{A} \\
\text { - Feb. 9, } 2009 \\
\text { - Forthcoming } \\
\text { in } 2009\end{array}$ \\
\hline \multicolumn{4}{|c|}{ B. Requests for work program inputs } \\
\hline $\begin{array}{l}\text { Fund request to } \\
\text { Bank }\end{array}$ & $\begin{array}{l}\text { - Growth diagnostic } \\
\text { - Assisting the authorities with food } \\
\text { strategy and scaling up agricultural } \\
\text { yield (An agriculture team is planning } \\
\text { to undertook an initial assessment of } \\
\text { the sector and reviewed the scope of } \\
\text { assistance/analysis needed). }\end{array}$ & $\begin{array}{ll}\text { - } & \text { On-going } \\
\text { - } & \text { August } 2008\end{array}$ & \\
\hline $\begin{array}{l}\text { Bank request to } \\
\text { Fund }\end{array}$ & $\begin{array}{l}\text { - Data and macro framework (including } \\
\text { the underlying analytical model). }\end{array}$ & - Ongoing & \\
\hline \multicolumn{4}{|c|}{ C. Agreements on joint products and missions } \\
\hline $\begin{array}{l}\text { Joint products } \\
\text { in next } 12 \\
\text { months }\end{array}$ & Low income debt sustainability analysis & Oct. 29-Nov. 11, 2008 & Feb. 9, 2009 \\
\hline
\end{tabular}




\section{Lesotho: Statistical ISSUES}

The statistical database is adequate for surveillance, although there are some deficiencies in core surveillance data including national accounts balance of payments statistics, and socio-economic data required to monitor progress toward the MDGs.

As one of 22 countries participating in the GDDS Project for Anglophone African Countries (funded by the U.K. Department for International Development (DFID)), Lesotho has undertaken the GDDS as framework for the development of its national statistical system. GDDS metadata have been posted on the Fund's Dissemination Standards Bulletin Board since August 2003 and were last updated - except national accounts - in the last quarter of 2007 .

\section{National accounts}

Revised national accounts were released in November 2008 by the Bureau of Statistics of Lesotho (BSL). Technical assistance from the EU and DFID supported the revision and the rebasing of the GDP data from 1981 to 2007. Important new sources of data include the use of VAT records and the 2002/03 household budget survey. Annual current and constant price (2004) estimates of GDP by economic activity, expenditure and income data are compiled by the BSL. These estimates have been published on the BSL's web site from 1998 through 2007. Other macroeconomic indicators, such as industrial production indices, are used to monitor developments during the year.

There is a need to improve and expand on the source data used for compiling national accounts. Although access to data from the VAT records has proved valuable in revising the national accounts, there is still a need for more detailed information on economic activities. Therefore, the business register kept by the BSL should be overhauled and updated accordingly.

\section{Prices}

Since November 2004, BSL produces a monthly consumer price index (CPI) that covers Maseru and ten other urban centers. While the index has a reference period of April 1997=100, the weights are still based on the 1994-95 HBS. In addition, a separate CPI is compiled and published for Maseru City. The 2002-03 HBS is yet to be used to derive new weights.

\section{Government finance statistics}

Data on government finance statistics are provided by the Ministry of Finance to AFR Department periodically, although timeliness of reports remains problematic. Current and capital expenditure would benefit from more disaggregated reporting, including for 
health and education; revenues should be classified according to the GFSM 2001; and the reporting lag for the functional breakdown of expenditure needs to be addressed. GFS should distinguish clearly between current and capital (project) grants and external loan disbursements.

A mission in 2004 assisted the authorities in implementing the reporting framework of GFSM 2001. The mission identified several shortcomings in the data. Underreporting of recurrent revenue and expenditure occurred as ministries net revenue against unreported expenditure. Reporting of acquisition of nonfinancial assets was incomplete as ministries do not fully report donor project outlays. These data were also insufficiently detailed to allow appropriate economic classifications.

\section{A subsequent mission in March 2006 found that progress with the implementation of the previous missions' recommendations had been impeded by lack of resources and high turnover of staff. More specifically, no progress has been observed in implementing the recommendation to expand the coverage of fiscal statistics to include extrabudgetary units of government, or in aligning the classification of transactions in the source accounting systems with GFSM 2001 classifications. A revision of the chart of accounts with the introduction of the Integrated Financial Management Information System has led to improvements in availability of source data.}

A follow up mission in 2007 helped the authorities to improve both annual and high frequency data for budgetary accounts. It also assisted with the analysis of local government accounts and made recommendation on the inclusion of data on extra-budgetary units in data for central government. However, the timeliness of data for these entities does not match the timeliness of budgetary central government data. Therefore, recently submitted 2005-06 data for publication in the Government Finance Statistical Yearbook 2007 was limited to budgetary central government, with a commitment to submit data on the other government units when these become available. No high frequency data are reported for publication in the International Finance Statistics.

\section{Monetary and financial statistics}

Lesotho compiles monetary statistics based on the Standardized Report Forms, which provide improved classification and sectorization of the accounts, and began reporting these data to the Statistics Department (STA) in December 2004. Monthly data following this new presentation are disseminated in the International Finance Statistics Supplement.

Despite the progress achieved, the authorities need, in line with STA recommendations, to expand the institutional coverage of the other depository corporations sector and to value all financial instruments at market value. A monetary and financial statistics mission in September 2007 found that work had started on the inventory of all financial corporations (deposit-taking and others), and that it was expected that credit cooperatives would be fully 
covered in the other depository corporations survey in last quarter in 2008. The compilation of the other financial corporations' survey is targeted for the first quarter in 2009.

\section{Balance of payments and external debt}

The CBL compiles quarterly balance of payments statistics, usually with a lag of three months. However, some timeliness issues remain in forwarding these data to the Fund.

A STA mission in mid-2002 found that the methodology underlying the compilation of the balance of payments data suffers from the use of outdated benchmark surveys and deterioration in the coverage and timeliness of some of data sources. The main recommendations of the mission were as follows:

- It is urgent to resolve both the inadequate coverage and the delayed reporting of data on exports and imports of goods. The Department of Customs and Excise (DCE) does not provide data in a timely fashion. The CBL, therefore, generates its own estimates and there are significant differences between these estimates and DCE's data. For example, CBL believes that DCE is underreporting total imports and has been adjusting the level of imports upward by 30 percent. There are also differences between the trade data compiled by the CBL and the BSL.

- Closer cooperation and new statistical surveys are needed to improve the quality of data on exports and imports of services. Most of the data on exports of services are based on outdated benchmark estimates adjusted for movements in the consumer price index. Data available at the ministries and other organizations could also be of better use for estimates of balance of payments variables. Government ministries and the CBL should cooperate more and make better use of data sources in organizations like the Lesotho Highlands Development Authority, the Lesotho National Development Corporation, the South African Reserve Bank, and the Employment Bureau of South Africa.

A STA mission in July-August 2008 made several recommendations. In general, the authorities need to strengthen their institutional capacity. The CBL should be given formal institutional responsibility for the compilation of balance of payments statistics. This work is currently undertaken by the CBL but there is no legislation or official decree that designates this responsibility. Together with other members of the Common Monetary Area, Lesotho's authorities also need to consider measures to harmonize concepts, methodology, and data collection for regional balance of payments data. 


\section{Lesotho: Table of Common Indicators Required for Surveillance}

(As of December 31, 2008)

\begin{tabular}{|c|c|c|c|c|c|}
\hline & 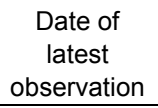 & $\begin{array}{l}\text { Date } \\
\text { received }\end{array}$ & $\begin{array}{c}\text { Frequency } \\
\text { of } \\
\text { Data 1/ }\end{array}$ & $\begin{array}{c}\text { Frequency } \\
\text { of } \\
\text { Reporting 1/ }\end{array}$ & $\begin{array}{l}\text { Frequency of } \\
\text { Publication } 1 /\end{array}$ \\
\hline Exchange rates & Dec. 2008 & Dec. 2008 & $\mathrm{D}$ & M & D \\
\hline $\begin{array}{l}\text { International reserve assets and reserve liabilities } \\
\text { of the monetary authorities } 2 /\end{array}$ & Sep 2008 & Oct. 2008 & M & Q & Q \\
\hline Reserve/base money & Sep. 2008 & Oct., 2008 & M & Q & Q \\
\hline Broad money & Sep. 2008 & Oct., 2008 & M & Q & Q \\
\hline Central bank balance sheet & Sep. 2008 & Oct., 2008 & M & Q & Q \\
\hline Consolidated balance sheet of the banking system & Sep. 2008 & Oct., 2008 & M & Q & Q \\
\hline Interest rates $3 /$ & Sep. 2008 & Oct., 2008 & M & M & M \\
\hline Consumer price index & Nov. 2008 & Oct. 2008 & M & M & M \\
\hline $\begin{array}{l}\text { Revenue, expenditure, balance and composition of } \\
\text { financing } 4 / \text {-general government } 5 /\end{array}$ & $\mathrm{N} / \mathrm{A}$ & N/A & N/A & $\mathrm{N} / \mathrm{A}$ & $\mathrm{N} / \mathrm{A}$ \\
\hline $\begin{array}{l}\text { Revenue, expenditure, balance and composition of } \\
\text { financing } 4 / \text {-central government }\end{array}$ & Sep. 2008 & Oct. 2008 & M & 1 & Q \\
\hline $\begin{array}{l}\text { Stocks of central government and central } \\
\text { government-guaranteed debt } 6 /\end{array}$ & Dec. 2007 & Cct. 2008 & M & 1 & Q \\
\hline External current account balance & Q2 2008 & Oct. 2008 & Q & Q & Q \\
\hline Exports and imports of goods and services & Q2 2008 & Oct. 2008 & Q & Q & Q \\
\hline GDP/GNP & 2007 & Oct 2008 & A & A & A \\
\hline Gross external debt & Dec. 2007 & Oct. 2008 & M & Q & Q \\
\hline International investment position $7 /$ & Q1 2008 & Oct. 2008 & Q & Q & Q \\
\hline
\end{tabular}

1/ Both market-based and officially-determined, including discount rates; money market rates; and rates on treasury bills, notes, and bonds.

2/ Foreign, domestic bank, and domestic nonbank financing.

$3 /$ The general government consists of the central government (budgetary funds, extra budgetary funds, and social security funds) and state and local governments.

4/ Including currency and maturity composition.

$5 /$ Includes external gross financial asset and liability positions vis-à-vis nonresidents.

6/Daily (D), weekly (W), monthly (M), quarterly (Q), annually (A), irregular (I); and not available.

$7 /$ Includes reserve assets pledged or otherwise encumbered as well as net derivative positions. 


\title{
INTERNATIONAL MONETARY FUND AND \\ INTERNATIONAL DEVELOPMENT ASSOCIATION
}

\author{
KINGDOM OF LESOTHO
}

\section{Joint World Bank/IMF Debt Sustainability Analysis}

Prepared by the staffs of the International Monetary Fund and the International Development Association

Approved by Robert Sharer and Anthony Boote (IMF) and Carlos A. Primo Braga and Sudhir Shetty (IDA)

January 23, 2009

This debt sustainability analysis (DSA) is based on end-2007 data for external and public debt provided by the Lesotho authorities, and World Bank and IMF staff estimates for debt outstanding to multilateral creditors. The overall staff assessment is that similar to last year's DSA, Lesotho is at a moderate risk of debt distress and remains vulnerable to adverse shocks to the exchange rate, Southern African Customs Union (SACU) revenues and the GDP growth rate, although debt appears manageable in a baseline scenario.

\section{INTRODUCTION}

1. This DSA has been prepared jointly by IMF and World Bank staff. It comprises external and domestic debt, and is based on the framework for low-income countries approved by the respective Executive Boards. The framework takes into account indicative thresholds for debt burden indicators determined by the quality of the country's policies and institutions, ${ }^{1}$ and comprises baseline and alternative scenarios.

2. Lesotho's nominal public sector debt has shown a significant decline since 2002 falling from 92 percent of GDP to 43 percent of GDP (US\$742.1 million) at the end of 2007. Much of the decline is attributed to the authorities' early repayment of non concessional loans, limit on new borrowing, and a significant exchange rate appreciation from 2003 to 2006 . Of the total public sector debt, US\$625.7 million was externally owed,

\footnotetext{
${ }^{1}$ The World Bank Country Policy and Institutional Assessment has ranked Lesotho using the three-year moving average as a "medium performer" in terms of policy and institutions with a rating of 3.5. The applicable indicative thresholds for debt sustainability, proposed under the framework for low-income countries are: (i) 40 percent for the NPV of debt-to-GDP ratio, (ii) 150 percent for NPV of debt-to-exports ratio; (iii) 250 percent for the NPV of debt-to-fiscal revenues ratio; (iv) 20 percent for the debt service to exports ratio; and (v) 30 percent for the debt service to revenue ratio.
} 
with about 92 percent of the total (US\$575.8 million) owed to multilateral creditors, mainly IDA and the African Development Fund. ${ }^{2}$ Government also has domestic debt held by residents in the amount of US\$116.4 million. For private sector debt, only obligations toward countries outside the Common Monetary Area are recorded. At end 2007, these obligations were estimated at US\$2.9 million.

\begin{tabular}{|c|c|c|}
\hline & $\begin{array}{l}\text { In Millions of } \\
\text { U.S. Dollars }\end{array}$ & $\begin{array}{r}\text { In Percent } \\
\text { of GDP }\end{array}$ \\
\hline Total debt & 745.0 & 43.1 \\
\hline Domestic and external ppg debt & 742.1 & 42.9 \\
\hline Domestic debt & 116.4 & 6.7 \\
\hline External public ppg debt & 625.7 & 36.2 \\
\hline Multilateral sources & 575.8 & 33.1 \\
\hline World Bank Group & 275.7 & 15.9 \\
\hline African Development Fund & 189.4 & 10.9 \\
\hline EU & 27.6 & 1.6 \\
\hline IMF & 32.2 & 1.9 \\
\hline Others & 50.9 & 2.9 \\
\hline Bilateral sources & 35.4 & 2.0 \\
\hline Commercial sources & 14.5 & 0.8 \\
\hline Total private external debt & 2.9 & 0.2 \\
\hline \multicolumn{3}{|l|}{ Memorandum item: } \\
\hline NPV of total external debt & 389.5 & 21.9 \\
\hline
\end{tabular}

\section{The global financial crisis and economic downturn will adversely affect}

Lesotho's economy. Lesotho's economy is affected by economic developments in its major trading partners, South Africa and the United States which account for 69 percent and 19 percent of its total trade, respectively. The main transmission channels include: (i) SACU revenues: A slower growth in South Africa's economy reduces import demand, hence, customs duties and excise revenues (the major sources of SACU revenues); SACU revenues have already been revised down by 6 percent of GDP in 2009; (ii) textiles: With the slowdown in the U.S. economy (Lesotho's main export market for garments), exports from Lesotho have declined by 15 percent during the first 10-months of 2008 and Lesotho has been losing market share in the U.S. textile market. In addition, as most of the textile

\footnotetext{
${ }^{2}$ The nominal public debt data does not include debt issued by the Lesotho Highlands Development Authority (LHDA). Under the terms of the agreement with South Africa, the liabilities of the LHDA, which are equal to about 10 percent of GDP, are not included since LHDA obtains financing to cover its debt service obligations from South Africa.
} 
factories in Lesotho are owned by firms in Asia, some of them are facing difficulties obtaining trade credit for input financing from their Asian banks, which have been affected by the global credit crunch; (iii) mining: weak prices for diamond and the global credit crunch have already affected mining production and weakened prospects in the near term; and (iv) worker remittances (20 percent of GDP) — mainly from South Africa-are likely to decline amid the slowdown in South Africa's mines which employ many Basothos.

4. Overall, real GDP growth is projected to slow down from 5.1 percent in 2007 to 3.9 percent and 2.1 percent in 2008 and 2009, respectively. Much of the slowdown in 2009 is attributable to falling mining and textile production, which are each projected to fall by 6 percent. However, as the global economy recovers, growth is expected to increase to a range of 4-5 percent in the medium term.

\section{Assumptions}

5. The baseline scenario is premised on a number of macroeconomic projections and reflects the global financial crisis and economic downturn in the near term (Box 1). Compared to last year's DSA, the 2008 DSA assumes a slightly lower rate of real GDP growth and a current account that is projected to be in deficit. Real GDP growth is now projected to average 4.1 percent up to 2013 with long-term growth remaining at 4.3 percent, compared to average growth rates of 5.3 in the medium-term projected in the last DSA. Sizable grants (US $\$ 362.5$ million, equivalent to 22 percent of GDP) will be made available to the government during the next five years under the Millennium Challenge Compact (MCC), which will allow it to undertake significant capital investment in the development of the country's health infrastructure, water supply, and private sector development. In particular, the construction of the Metolong dam is expected to increase the potential for the location of "wet industries" such as fabric production, which would allow Lesotho to take fuller advantage of the African Growth and Opportunity Act (AGOA) beyond 2012. Overall, this is expected to contribute around 1.5 percent to growth in the medium term. In addition, the recent approval by South Africa of the second phase of the LHDA water project scheduled to begin in 2012/13, will help in contributing to the slightly better medium-term growth performance than has been observed in the past. However, the recent problems in the mining and manufacturing sectors are expected to adversely affect growth and as such the projections for this year's DSA are somewhat lower than last year's.

\section{The 2008 DSA assumes the current account will return to deficit as the level of} SACU transfers drop to a lower level and the textile and mining sectors come under increasing pressure. After a few years of good performance the textile sector has begun to experience a decline. In addition, diamond exports and revenue from the SACU are projected to be lower and should adversely affect the current account in the medium term. Under the 


\section{Box 1. Main Assumptions Under the Baseline Scenario}

- $\quad$ Real GDP growth is assumed to be supported by the implementation of projects under the MCC and the second phase of the LHDA water project. Growth should increase from 3.3 percent over the last ten years to about 4.1 percent in 2008-13 and then from 2014 to stabilize at 4.3 percent.

- Inflation (as measured by the implicit GDP deflator) is assumed to move from an average of about $7 \frac{1}{2}$ percent over the last ten years to about 6 percent over the medium term and falling to about 4 percent over the longer term. This is in line with anticipated inflation developments in South Africa.

- $\quad$ Despite lower SACU revenues, the overall fiscal balance is projected to be in surplus over the medium term (5 percent of GDP) consistent with a stable non-SACU deficit of 24-25 percent of GDP. Over the long term, the fiscal surplus is projected to narrow down to below 4 percent of GDP, with revenues growing in line with nominal GDP and primary spending constant in real terms.

- $\quad$ Growth in exports of goods and services (in U.S. dollar terms) after averaging 14 percent over the last 10 years is assumed to drop on average by 3 percent over the medium term before recovering to about 8 percent in the long term. Import growth is assumed at about 2 percent over the medium term before climbing to more than 8 percent in the longer term broadly in line with GDP growth.

- The current account balance (including official transfers) is determined by the above trends, declining from a 12.7 percent of GDP surplus in 2007 to subsequent deficits as SACU transfers, textile and diamond exports decline significantly. Net income is also assumed to decrease gradually over the long term as remittances from South Africa continue to become less important over time.

- $\quad$ Net external public sector financing rises gradually, peaking at 3 percent of GDP by 2015 and stabilizes at about $1 \frac{1}{2}$ percent of GDP over the long term. Foreign grants are assumed to increase to about 5 percent of GDP over the medium term, reflecting the MCC compact, and thereafter to decline to about $1 \frac{1}{2}$ percent of GDP. After 2012, it is assumed that borrowing from IDA would be at hardened terms. It is further assumed that new non-IDA borrowing would be contracted on highly concessional terms

- Domestic debt is projected to fall in nominal terms gradually over time. Private sector debt is projected to increase only marginally in terms of GDP, to 0.5 percent by 2028 .

Atlas method, Lesotho's GNI per capita stood at US\$1,000 in 2007. Taking into account the lags included in this methodology and the assumptions about growth, Lesotho would no longer be able to borrow at standard IDA terms after 2010. ${ }^{3}$ In the baseline scenario, Lesotho starts facing "IDA-hardened" terms after 2012 as a result of the growth in its GNI per capita.

\footnotetext{
${ }^{3}$ In the DSA, IDA-hardened terms are incorporated and substituted for standard IDA terms after 2012, and implies a charge of 0.75 percent, grace period of 10 years and a maturity period of 20 years (including the grace period), compared to the 40-year maturity of standard IDA terms.
} 
Comparison of Key Variables in Debt Sustainability Analysis 2006-13

\begin{tabular}{|c|c|c|c|c|c|c|c|c|}
\hline & \multicolumn{4}{|c|}{ Real GDP Growth } & \multicolumn{4}{|c|}{ Noninterest Current Account (In Percent of GDP) } \\
\hline & 2006 & 2007 & 2008 & Actual & 2006 & 2007 & 2008 & Actual \\
\hline 2006 & 2.5 & $\ldots$ & $\ldots$ & 8.1 & -3.2 & $\ldots$ & $\ldots$ & 4.9 \\
\hline 2007 & 1.4 & 4.9 & $\ldots$ & 5.1 & 1.8 & 2.1 & $\ldots$ & 16.7 \\
\hline 2008 & 1.4 & 5.2 & 3.9 & $\ldots$ & -1.4 & 1.2 & -3.4 & $\ldots$ \\
\hline 2009 & 2.6 & 5.4 & 2.1 & $\ldots$ & -4.7 & 2.0 & -8.1 & $\ldots$ \\
\hline 2010 & 2.7 & 5.5 & 5.5 & $\ldots$ & -6.1 & 1.1 & -6.1 & $\ldots$ \\
\hline 2011 & 2.7 & 5.6 & 4.5 & $\ldots$ & -6.5 & -0.8 & -5.1 & $\ldots$ \\
\hline 2012 & 2.8 & 5.1 & 4.3 & $\ldots$ & -7.3 & -1.7 & -4.9 & $\ldots$ \\
\hline 2013 & 3.0 & 5.2 & 4.2 & $\ldots$ & -8.1 & -2.1 & -4.5 & $\ldots$ \\
\hline
\end{tabular}

\section{EXTERNAL DEBT SUSTAINABILITY}

\section{Baseline}

7. At end-2007, the NPV of external debt stood at 21.9 percent of GDP (Table 1a). Under the baseline scenarios, Lesotho's external debt indicators remain well below the thresholds throughout the projection period. The PV of debt-to-GDP ratio is expected to gradually decrease to 20 percent in 2018 , below the policy-based indicative threshold (40 percent); and the PV of debt-to-export ratio would rise to 64 percent by 2018, although it would still be significantly below the 150 percent threshold. The highly concessional nature of the existing debt and new borrowing contributes to debt service ratios below the indicative threshold throughout the projection period. The government undertook to repay early a significant amount of non concessional debt resulting in lower scheduled interest payments and hence a declining effective interest on debt. Borrowing, however, is expected to still be on broadly concessional terms. ${ }^{4}$

\section{Alternative scenarios and stress tests}

8. Sensitivity tests show that while Lesotho's debt burden would worsen in the event of an adverse macroeconomic shock or weaker economic performance compared to historical outcomes, it would remain below the indicative thresholds in most cases (Table 1b and Figure 1). Real GDP growth in the 10-year period up to 2007 averaged 3.3 percent, less than that assumed in the projection period. The noninterest current account was also significantly weaker than that projected under the baseline scenario due to the lower level of SACU transfers. As a result, the evolution of debt would be adversely affected should the key macroeconomic variables during the projection period revert to their pre-2007 levels. Debt indicators in the historical scenario (scenario A1) tend to be more elevated than

\footnotetext{
4 The depreciation of the loti had a significant effect on the debt-to-GDP ratio in 2008.
} 
under the baseline scenario, with some indicators (PV of debt to GDP and PV of debt to exports) crossing the debt thresholds by 2020, underscoring the need to become more competitive, boost export receipts and grow at a faster rate.

9. A shock to SACU transfers could be sizable. In October 2008, SACU revenue projection for Lesotho was revised down by about 6 percent of GDP relative to the previous projection for 2009/10. The baseline scenario already incorporates this downward revision. The sensitivity scenario of reducing SACU transfers thus assumes a permanent decline of the same magnitude ( 6 percent of GDP) relative to the baseline for the entire projection period starting in 2009. The same sensitivity exercise is also conducted for public debt DSA; see below. SACU transfers represent more than 50 percent of export receipts. When SACU transfers are reduced permanently relative to the baseline by about 6 percent of GDP, (Table $1 \mathrm{~b}$; scenario A3), all debt indicators worsen. More importantly, two debt indicators (PV of debt to GDP and PV of debt to exports) cross their thresholds as early as 2014 and 2016, respectively, underscoring the importance of these transfers to sustainability of the current account.

10. The bound tests reveal that Lesotho would face the most distress if there were to be a much lower level of non debt creating flows (scenario B1). Under this scenario, the PV of debt-to-GDP ratio first increases to 40 percent of GDP in 2010 before falling to 33 percent of GDP in 2018. There are also underlying vulnerabilities with respect to the combined impact of lower GDP, export growth and nondebt creating flows. Such a shock would lead to higher PV of debt-to-GDP and PV of debt-to- exports ratios compared to the baseline scenario.

\section{Public Sector Debt Sustainability}

\section{Baseline}

11. At end-2007 domestic debt contributed only marginally to the baseline scenarios for Lesotho's public debt ratios (Table 2a). Lesotho has a low level of domestic debt, and so public debt indicators are very closely aligned to those of public external debt. Domestic debt, which was at 6.7 percent of GDP at the end of 2007, is expected to be gradually reduced by 2018 to 3 percent of GDP. Domestic debt has been issued by government mostly to provide an impetus to the formation of a money market. The proceeds from the sale of Tbills are held in deposits at the Central Bank of Lesotho; therefore these bills have not represented additions to net debt.

\section{The trajectory of gross debt understates somewhat the decline in Lesotho's net}

indebtedness especially in the next few years. This trajectory takes into account the disbursement of loans in the pipeline even in periods when fiscal surpluses would permit a net reduction of debt. It is also assumed that the authorities will stick to the original schedule for the repayment of the highly concessional debt. This implies that the public sector will 
accumulate some assets over the medium term, as indicated by large residuals in tables 1a and 2a. Alternatively, the analysis could be assumed to show that there is margin for lower primary surpluses that would remain consistent with the reduction in gross debt shown in Figure 2. However, because of limited implementation capacity, the authorities are not expected to significantly increase their level of capital investment making it more likely that they will accumulate assets rather than significantly increase their investment levels.

\section{Alternative scenarios and stress tests}

13. In the standard sensitivity tests public sector debt to GDP ratios will deteriorate (Table 2b and Figure 2). The indicators are most sensitive to a permanent decline in SACU revenues and deviations from the baseline growth path. In the scenario with variables at historical averages, ratios initially rise but eventually decline. The most extreme shock is linked to a permanent decline in SACU revenues further reinforcing the need to guard fiscal sustainability.

\section{A permanent adverse shock to SACU revenues could lead to a rising public debt} profile. A shock of the same magnitude as in the external DSA (sustained reduction in SACU revenue) would increase the PV of public debt to GDP ratio from 31 percent in 2009 to 40 percent in 2013, a sizable increase over only five years. By 2028, the ratio would stand at 68 percent, twice the ratio in 2008 .

\section{Conclusion}

\section{Lesotho faces a moderate risk of debt distress although in the baseline scenario} key debt ratios are below the indicative thresholds for a country with Lesotho's performance rating. The risk would materialize if key variables such as economic growth and the current account deficit were to revert to their historical levels or if Lesotho were to face a sizable adverse shock to SACU revenues. These results therefore underscore the need for the authorities to enhance non-SACU revenues, contain overall spending, ensure adequate levels of reserves, and accelerate growth, through increased competitiveness while continuing to seek grants and highly concessional loans. 
Table 1a. Lesotho: External Debt Sustainability Framework, Baseline Scenario, 2005-2028 1/

(In percent of GDP; unless otherwise indicated)

\begin{tabular}{|c|c|c|c|c|c|c|c|c|c|c|c|c|c|c|c|}
\hline & \multicolumn{3}{|c|}{ Actual } & \multirow{2}{*}{$\begin{array}{l}\text { Historical } \\
\text { Average }\end{array}$} & \multirow{2}{*}{$\begin{array}{l}\text { Standard } \\
\text { Deviation }\end{array}$} & \multicolumn{5}{|c|}{ Projections } & \multicolumn{3}{|c|}{$2008-2013$} & \multicolumn{2}{|r|}{$2014-2028$} \\
\hline & 2005 & 2006 & 2007 & & & 2008 & 2009 & 2010 & 2011 & 2012 & 2013 & Average & 2018 & 2028 & Average \\
\hline External debt (nominal) $1 /$ & 45.4 & 42.3 & 36.3 & & & 46.9 & 38.5 & 35.5 & 33.4 & 32.4 & 31.9 & & 31.6 & 24.9 & \\
\hline Ot which: Public and publicly guaranteed (PPG) & 45.2 & 42.1 & 36.2 & & & 46.7 & 38.3 & 35.3 & 33.2 & 32.1 & 31.6 & & 31.4 & 24.4 & \\
\hline Change in external debt & -3.0 & -3.1 & -6.0 & & & 10.6 & -8.5 & -2.9 & -2.1 & -1.0 & -0.5 & & -0.3 & -0.2 & \\
\hline Identified net debt-creating flows & 0.2 & -14.6 & -22.9 & & & -3.1 & 3.8 & 0.0 & -1.2 & -1.9 & -2.2 & & -1.6 & -1.8 & \\
\hline Noninterest current account deficit & 6.5 & -4.9 & -16.7 & 8.1 & 12.7 & 3.4 & 8.1 & 6.1 & 5.1 & 4.9 & 4.5 & & 5.4 & 6.0 & 5.2 \\
\hline Deficit in balance of goods and services & 51.4 & 46.4 & 49.8 & & & 63.4 & 57.8 & 53.7 & 53.4 & 52.6 & 51.8 & & 44.5 & 41.6 & \\
\hline Exports & 51.1 & 50.0 & 52.7 & & & 47.3 & 41.6 & 38.7 & 36.5 & 34.4 & 32.6 & & 31.0 & 31.3 & \\
\hline Imports & 102.5 & 96.4 & 102.5 & & & 110.8 & 99.4 & 92.4 & 90.0 & 86.9 & 84.4 & & 75.5 & 72.9 & \\
\hline Net current transfers (negative $=$ inflow) & -21.8 & -25.7 & -37.6 & -21.2 & 6.3 & -33.8 & -28.5 & -27.8 & -29.3 & -29.1 & -28.9 & & -21.2 & -10.3 & -17.9 \\
\hline Of which: Official & -21.1 & -24.5 & -37.5 & & & -33.7 & -28.4 & -27.8 & -29.2 & -29.1 & -28.9 & & -27.8 & -29.0 & \\
\hline Other current account flows (negative $=$ net inflow) & -23.1 & -25.7 & -29.0 & & & -26.2 & -21.2 & -19.8 & -19.0 & -18.6 & -18.4 & & -17.9 & -25.2 & \\
\hline Net FDI (negative $=$ inflow) & -4.2 & -6.1 & -6.3 & -4.4 & 1.0 & -5.4 & -3.9 & -4.6 & -5.3 & -5.9 & -5.9 & & -6.3 & -7.3 & -6.6 \\
\hline Endogenous debt dynamics $2 /$ & -2.1 & -3.6 & 0.2 & & & -1.2 & -0.4 & -1.5 & -1.0 & -0.9 & -0.8 & & -0.8 & -0.6 & \\
\hline Contribution from nominal interest rate & 0.9 & 0.6 & 4.1 & & & 0.3 & 0.5 & 0.4 & 0.5 & 0.5 & 0.5 & & 0.5 & 0.4 & \\
\hline Contribution from real GDP growth & -0.3 & -3.3 & -1.9 & & & -1.5 & -0.9 & -1.9 & -1.5 & -1.4 & -1.3 & & -1.3 & -1.0 & \\
\hline Contribution from price and exchange rate changes & -2.7 & -0.9 & -2.0 & & & & & & & & & & & & \\
\hline Residual (3-4) $3 /$ & -3.2 & 11.5 & 16.9 & & & 13.7 & -12.2 & -3.0 & -0.9 & 0.9 & 1.7 & & 1.4 & 1.6 & \\
\hline Of which: Exceptional tinancing & 0.0 & 0.0 & 0.0 & & & 0.0 & 0.0 & 0.0 & 0.0 & 0.0 & 0.0 & & 0.0 & 0.0 & \\
\hline PV of external debt $4 /$ & $\ldots$ & $\ldots$ & 21.9 & & & 23.3 & 22.8 & 21.4 & 20.3 & 19.8 & 19.6 & & 19.8 & 16.5 & \\
\hline In percent of exports & $\ldots$ & $\ldots$ & 41.5 & & & 49.2 & 55.0 & 55.2 & 55.6 & 57.7 & 60.2 & & 64.0 & 52.7 & \\
\hline PV of PPG external debt & $\ldots$ & $\ldots$ & 21.7 & & & 23.1 & 22.7 & 21.2 & 20.1 & 19.6 & 19.4 & & 19.7 & 16.0 & \\
\hline In percent of exports & $\ldots$ & $\ldots$ & 41.2 & & & 48.8 & 54.5 & 54.6 & 55.0 & 57.0 & 59.5 & & 63.5 & 51.1 & \\
\hline In percent of government revenues & $\ldots$ & $\ldots$ & 34.2 & & & 38.0 & 41.9 & 40.3 & 37.2 & 36.2 & 35.8 & & 36.7 & 29.8 & \\
\hline Debt service-to-exports ratio (in percent) & 11.6 & 5.4 & 11.5 & & & 2.8 & 4.5 & 4.5 & 4.9 & 4.8 & 5.0 & & 4.4 & 4.7 & \\
\hline PPG debt service-to-exports ratio (in percent) & 11.6 & 5.4 & 11.5 & & & 2.8 & 4.5 & 4.5 & 4.9 & 4.8 & 5.0 & & 4.4 & 4.7 & \\
\hline PPG debt service-to-revenue ratio (in percent) & 11.7 & 4.7 & 9.5 & & & 2.2 & 3.5 & 3.3 & 3.3 & 3.0 & 3.0 & & 2.5 & 2.7 & \\
\hline Total gross financing need (billions of U.S. dollars) & 0.1 & -0.1 & -0.3 & & & 0.0 & 0.1 & 0.1 & 0.0 & 0.0 & 0.0 & & 0.0 & 0.0 & \\
\hline Noninterest current account deficit that stabilizes debt ratio & 9.5 & -1.8 & -10.8 & & & -7.1 & 16.6 & 9.0 & 7.2 & 5.9 & 5.0 & & 5.7 & 6.2 & \\
\hline Key macroeconomic assumptions & & & & & & & & & & & & & & & \\
\hline Real GDP growth (in percent) & 0.7 & 8.1 & 5.1 & 3.3 & 2.4 & 3.9 & 2.1 & 5.5 & 4.5 & 4.3 & 4.2 & 4.1 & 4.3 & 4.3 & 4.3 \\
\hline GDP deflator in US dollar terms (change in percent) & 6.0 & 2.0 & 4.9 & 4.0 & 17.2 & -6.7 & 3.2 & 4.3 & 1.8 & 1.8 & 1.3 & 1.0 & 4.0 & 4.0 & 4.0 \\
\hline Effective interest rate (percent) 5/ & 2.0 & 1.4 & 10.7 & 3.1 & 2.7 & 0.8 & 1.2 & 1.3 & 1.4 & 1.4 & 1.5 & 1.3 & 1.6 & 1.8 & 1.6 \\
\hline Growth of exports of G\&S (US dollar terms, in percent) & -9.2 & 7.9 & 16.0 & 14.0 & 20.1 & -12.8 & -7.4 & 2.6 & 0.3 & 0.0 & 0.1 & -2.9 & 8.6 & 8.2 & 8.2 \\
\hline Growth of imports of G\&S (US dollar terms, in percent) & 1.5 & 3.6 & 17.2 & 5.7 & 16.6 & 4.9 & -5.4 & 2.4 & 3.6 & 2.7 & 2.5 & 1.8 & 7.8 & 8.3 & 7.4 \\
\hline Grant element of new public sector borrowing (in percent) & & $\ldots$ & & $\ldots$ & $\ldots$ & 22.2 & 34.0 & 30.6 & 31.1 & 35.9 & 34.1 & 31.3 & 37.2 & 29.7 & 34.7 \\
\hline Government revenues (excluding grants, in percent of GDP) & 50.4 & 57.6 & 63.5 & & & 60.7 & 54.0 & 52.5 & 53.9 & 54.1 & 54.2 & & 53.7 & 53.7 & 53.7 \\
\hline Aid flows (in Billions of US dollars) $7 /$ & 0.0 & 0.0 & 0.0 & & & 0.0 & 0.1 & 0.1 & 0.1 & 0.1 & 0.1 & & 0.0 & 0.1 & \\
\hline Of which: Grants & 0.0 & 0.0 & 0.0 & & & 0.0 & 0.1 & 0.1 & 0.1 & 0.1 & 0.1 & & 0.0 & 0.1 & \\
\hline Of which: Concessional loans & 0.0 & 0.0 & 0.0 & & & 0.0 & 0.0 & 0.0 & 0.0 & 0.0 & 0.0 & & 0.0 & 0.0 & \\
\hline Grant-equivalent financing (in percent of GDP) $8 /$ & $\ldots$ & $\ldots$ & $\ldots$ & & & 2.3 & 4.5 & 5.4 & 6.2 & 5.7 & 3.7 & & 2.4 & 2.0 & 2.3 \\
\hline Grant-equivalent financing (in percent of external financing) $8 /$ & $\ldots$ & $\ldots$ & $\ldots$ & & & 72.7 & 74.5 & 80.5 & 86.6 & 80.4 & 70.8 & & 55.8 & 54.1 & 55.9 \\
\hline Memorandur & & & & & & & & & & & & & & & \\
\hline Nominal GDP (billions of U.S. dollars) & 1.4 & 1.5 & 1.7 & & & 1.6 & 1.7 & 1.9 & 2.0 & 2.1 & 2.2 & & 3.4 & 7.6 & \\
\hline Nominal dollar GDP growth & 6.7 & 10.2 & 10.2 & & & -3.0 & 5.4 & 10.1 & 6.4 & 6.2 & 5.6 & 5.1 & 8.5 & 8.5 & 8.5 \\
\hline PV of PPG external debt (in billions of U.S. dollars) & & & 0.4 & & & 0.4 & 0.4 & 0.4 & 0.4 & 0.4 & 0.4 & & 0.7 & 1.2 & \\
\hline (PVt-PVt-1)/GDPt-1 (in percent) & & & & & & 0.7 & 0.8 & 0.6 & 0.2 & 0.7 & 0.9 & 0.7 & 1.6 & 1.1 & 1.3 \\
\hline
\end{tabular}

\section{Source: Staff simulations.}

$1 /$ Includes both public and private sector external debt.

2/ Derived as $[r-g-r(1+g)] /(1+g+r+g r)$ times previous period debt ratio, with $r=$ nominal interest rate; $g=$ real GDP growth rate, and $r=$ growth rate of GDP deflator in U.S. dollar terms.

3 / Includes exceptional financing (i.e., changes in arrears and debt relief); changes in gross foreign assets; and valuation adjustments. For projections also includes contribution from price and exchange rate changes.

4/ Assumes that PV of private sector debt is equivalent to its face value.

6/ Historical averages and standard deviations are generally derived over the past 10 years, subject to data availability.

7/ Defined as grants, concessional loans, and debt relief.

8/ Grant-equivalent financing includes grants provided directly to the government and through new borrowing (difference between the face value and the PV of new debt). 


\section{Table 1b.Lesotho: Sensitivity Analysis for Key Indicators of Public and Publicly Guaranteed External Debt, 2008-2028}

(Projections; in percent)

\begin{tabular}{llllllll}
2008 & 2009 & 2010 & 2011 & 2012 & 2013 & 2018 & 2028 \\
\hline
\end{tabular}

Baseline

PV of debt-to GDP ratio

A. Alternative Scenarios

A1. Key variables at their historical averages in 2008-2028 1/

A2. New public sector loans on less favorable terms in 2008-2028 2/

A3.Permanent decline in SACU transfers by 6 percent of GDP starting in 2009

$\begin{array}{llllllll}23 & 23 & 21 & 20 & 20 & 19 & 20 & 16\end{array}$

B. Bound Tests

B1. Real GDP growth at historical average minus one standard deviation in 2009-2010

B2. Export value growth at historical average minus one standard deviation in 2009-2010 3/

B3. US dollar GDP deflator at historical average minus one standard deviation in 2009-2010

B4. Net non-debt creating flows at historical average minus one standard deviation in 2009-2010 4/

B5. Combination of B1-B4 using one-half standard deviation shocks

B6. One-time 30 percent nominal depreciation relative to the baseline in 2009 5/

Baseline

A. Alternative Scenarios

A1. Key variables at their historical averages in 2008-2028 $1 /$

A2. New public sector loans on less favorable terms in 2008-2028 $2 /$

A3.Permanent decline in SACU transfers by 6 percent of GDP starting in 2009

B. Bound Tests

B1. Real GDP growth at historical average minus one standard deviation in 2009-2010

B2. Export value growth at historical average minus one standard deviation in 2009-2010 3/

B3. US dollar GDP deflator at historical average minus one standard deviation in 2009-2010

B4. Net non-debt creating flows at historical average minus one standard deviation in 2009-2010 4/

B5. Combination of B1-B4 using one-half standard deviation shocks

B6. One-time 30 percent nominal depreciation relative to the baseline in 2009 5/

$\begin{array}{llllllll}23 & 21 & 21 & 22 & 24 & 27 & 39 & 51 \\ 23 & 23 & 22 & 21 & 21 & 21 & 22 & 20 \\ 23 & 27 & 29 & 32 & 36 & 39 & 54 & 63\end{array}$

$\begin{array}{llllllll}23 & 23 & 22 & 21 & 20 & 20 & 21 & 17 \\ 23 & 21 & 19 & 18 & 18 & 18 & 18 & 16 \\ 23 & 26 & 29 & 28 & 27 & 27 & 27 & 22 \\ 23 & 32 & 40 & 39 & 38 & 37 & 33 & 20 \\ 23 & 28 & 33 & 32 & 31 & 30 & 29 & 20 \\ 23 & 32 & 30 & 28 & 28 & 27 & 28 & 22\end{array}$

PV of debt-to-exports ratio

$\begin{array}{llllllll}49 & 55 & 55 & 55 & 57 & 60 & 63 & 51\end{array}$

Baseline

\section{A. Alternative Scenarios}

A1. Key variables at their historical averages in 2008-2028 1/

A2. New public sector loans on less favorable terms in 2008-2028 2/

A3.Permanent decline in SACU transfers by 6 percent of GDP starting in 2009

$\begin{array}{rrrrrrrr}49 & 54 & 54 & 55 & 57 & 59 & 63 & 51 \\ 49 & 48 & 50 & 50 & 52 & 54 & 59 & 50 \\ 49 & 54 & 54 & 55 & 57 & 59 & 63 & 51 \\ 49 & 78 & 103 & 106 & 110 & 114 & 106 & 63 \\ 49 & 54 & 60 & 60 & 63 & 65 & 65 & 46 \\ 49 & 54 & 54 & 55 & 57 & 59 & 63 & 51\end{array}$

B. Bound Tests

B1. Real GDP growth at historical average minus one standard deviation in 2009-2010

B2. Export value growth at historical average minus one standard deviation in 2009-2010 3/

B3. US dollar GDP deflator at historical average minus one standard deviation in 2009-2010

B4. Net non-debt creating flows at historical average minus one standard deviation in 2009-2010 4/

B5. Combination of B1-B4 using one-half standard deviation shocks

B6. One-time 30 percent nominal depreciation relative to the baseline in 2009 5/

PV of debt-to-revenue ratio

$\begin{array}{llllllll}38 & 42 & 42 & 39 & 38 & 37 & 38 & 31 \\ 38 & 39 & 37 & 34 & 33 & 33 & 34 & 29 \\ 38 & 49 & 56 & 52 & 50 & 50 & 51 & 41 \\ 38 & 60 & 76 & 71 & 70 & 69 & 61 & 37 \\ 38 & 51 & 63 & 58 & 57 & 56 & 54 & 38 \\ 38 & 59 & 57 & 52 & 51 & 50 & 51 & 42\end{array}$




\section{Table 1b.Lesotho: Sensitivity Analysis for Key Indicators of Public and Publicly Guaranteed External Debt, 2008-2028}

(Projections; in percent)

\begin{tabular}{llllllll}
2008 & 2009 & 2010 & 2011 & 2012 & 2013 & 2018 & 2028 \\
\hline
\end{tabular}

Baseline

Debt service-to-exports ratio

$\begin{array}{llllllll}3 & 5 & 5 & 5 & 5 & 5 & 4 & 5\end{array}$

A. Alternative Scenarios

A1. Key variables at their historical averages in 2008-2028 1/

A2. New public sector loans on less favorable terms in 2008-2028 2/

A3.Permanent decline in SACU transfers by 6 percent of GDP starting in 2009

$\begin{array}{lllllll}5 & 5 & 5 & 5 & 5 & 4\end{array}$

B. Bound Tests

B1. Real GDP growth at historical average minus one standard deviation in 2009-2010

B2. Export value growth at historical average minus one standard deviation in 2009-2010 3/

B3. US dollar GDP deflator at historical average minus one standard deviation in 2009-2010

B4. Net non-debt creating flows at historical average minus one standard deviation in 2009-2010 4/

B5. Combination of B1-B4 using one-half standard deviation shocks

B6. One-time 30 percent nominal depreciation relative to the baseline in 2009 5/

\begin{tabular}{|c|c|c|c|c|c|}
\hline 4 & 4 & 5 & 5 & 5 & 6 \\
\hline 5 & 5 & 5 & 5 & 5 & 5 \\
\hline 5 & 5 & 6 & 6 & 6 & 8 \\
\hline
\end{tabular}

Baseline

$\begin{array}{lllllll}5 & 5 & 5 & 5 & 5 & 4 & 5 \\ 4 & 4 & 5 & 5 & 5 & 4 & 5 \\ 5 & 5 & 5 & 5 & 5 & 4 & 5 \\ 5 & 5 & 6 & 6 & 7 & 8 & 7 \\ 4 & 4 & 4 & 4 & 5 & 5 & 4 \\ 5 & 5 & 5 & 5 & 5 & 4 & 5\end{array}$

A. Alternative Scenarios

A1. Key variables at their historical averages in 2008-2028 1/

A2. New public sector loans on less favorable terms in 2008-2028 2/

A3.Permanent decline in SACU transfers by 6 percent of GDP starting in 2009

Debt service-to-revenue ratio

\section{B. Bound Tests}

B1. Real GDP growth at historical average minus one standard deviation in 2009-2010

B2. Export value growth at historical average minus one standard deviation in 2009-2010 3/

B3. US dollar GDP deflator at historical average minus one standard deviation in 2009-2010

B4. Net non-debt creating flows at historical average minus one standard deviation in 2009-2010 4/

B5. Combination of B1-B4 using one-half standard deviation shocks

B6. One-time 30 percent nominal depreciation relative to the baseline in 2009 5/

Memorandum item:

Grant element assumed on residual financing (i.e., financing required above baseline) 6/

$\begin{array}{llllllll}2 & 3 & 4 & 3 & 3 & 3 & 3 & 3 \\ 2 & 3 & 3 & 3 & 3 & 3 & 2 & 3 \\ 2 & 4 & 5 & 5 & 4 & 4 & 3 & 4 \\ 2 & 3 & 4 & 4 & 4 & 4 & 5 & 4 \\ 2 & 4 & 4 & 4 & 4 & 4 & 4 & 4 \\ 2 & 5 & 5 & 5 & 4 & 4 & 4 & 4 \\ 30 & 30 & 30 & 30 & 30 & 30 & 30 & 30\end{array}$

Source: Staff projections and simulations.

1/ Variables include real GDP growth, growth of GDP deflator (in U.S. dollar terms), non-interest current account in percent of GDP, and non-debt creating flows.

2/ Assumes that the interest rate on new borrowing is by 2 percentage points higher than in the baseline, while grace and maturity periods are the same as in the baseline.

3/ Exports values are assumed to remain permanently at the lower level, but the current account as a share of GDP is assumed to return to its baseline level after the shock (implicitly assuming an offsetting adjustment in import levels).

4 / Includes official and private transfers and FDI.

5/ Depreciation is defined as percentage decline in dollar/local currency rate, such that it never exceeds 100 percent.

6/ Applies to all stress scenarios except for A2 (less favorable financing) in which the terms on all new financing are as specified in footnote 2. 
Table 2a. Lesotho: Public Sector Debt Sustainability Framework, Baseline Scenario, 2005-2028

(In percent of GDP, unless otherwise indicated)

\begin{tabular}{|c|c|c|c|c|c|c|c|c|c|c|c|c|c|c|c|}
\hline & \multirow{2}{*}{\multicolumn{3}{|c|}{ Actual }} & \multirow[b]{3}{*}{ Average } & \multirow{3}{*}{$\begin{array}{l}\text { Standard } \\
\text { Deviation }\end{array}$} & \multicolumn{10}{|c|}{ Projections } \\
\hline & & & & & & \multirow[b]{2}{*}{2008} & \multirow[b]{2}{*}{2009} & \multirow[b]{2}{*}{2010} & \multirow[b]{2}{*}{2011} & \multirow[b]{2}{*}{2012} & \multicolumn{2}{|c|}{$2008-13$} & \multirow[b]{2}{*}{2018} & \multirow[b]{2}{*}{2028} & \multirow{2}{*}{$\begin{array}{c}2014-28 \\
\text { Average }\end{array}$} \\
\hline & 2005 & 2006 & 2007 & & & & & & & & 2013 & Average & & & \\
\hline Public sector debt $1 /$ & 54.5 & 50.3 & 42.9 & & & 52.4 & 43.1 & 39.6 & 37.3 & 36.1 & 35.5 & & 34.7 & 26.7 & \\
\hline Ut which: roreign-currency denominated & 45.2 & 42.1 & 36.2 & & & 46.7 & 38.3 & 35.3 & 33.2 & 32.1 & 31.6 & & 31.4 & 24.4 & \\
\hline Change in public sector debt & -2.9 & -4.3 & -7.4 & & & 9.4 & -9.3 & -3.4 & -2.3 & -1.2 & -0.6 & & -0.4 & -0.6 & \\
\hline Identified debt-creating flows & -2.1 & -16.9 & -24.0 & & & 0.8 & -13.3 & -8.0 & -8.3 & -8.3 & -7.8 & & -6.4 & -5.8 & \\
\hline Primary deficit & -6.6 & -13.8 & -20.9 & -4.0 & 9.2 & -9.6 & -4.2 & -4.6 & -6.4 & -6.6 & -6.4 & -6.3 & -4.3 & -4.2 & -4.2 \\
\hline Revenue and grants & 52.6 & 58.7 & 64.8 & & & 62.8 & 57.7 & 57.3 & 59.7 & 59.0 & 57.1 & & 55.0 & 55.0 & \\
\hline Of which: Grants & 2.2 & 1.1 & 1.3 & & & 2.0 & 3.7 & 4.8 & 5.8 & 4.9 & 2.9 & & 1.3 & 1.3 & \\
\hline Primary (noninterest) expenditure & 46.0 & 44.9 & 43.9 & & & 53.2 & 53.6 & 52.8 & 53.4 & 52.5 & 50.7 & & 50.7 & 50.8 & \\
\hline Automatic debt dynamics & 4.5 & -3.1 & -3.0 & & & 10.4 & -9.1 & -3.4 & -1.9 & -1.8 & -1.3 & & -2.1 & -1.6 & \\
\hline Contribution from interest rate/growth differential & -0.8 & -5.0 & 0.5 & & & -2.0 & -1.5 & -2.6 & -1.8 & -1.7 & -1.6 & & -1.5 & -1.2 & \\
\hline Ut which: Contribution trom average real interest rate & -0.4 & -1.0 & 3.0 & & & -0.4 & -0.4 & -0.3 & -0.1 & -0.1 & -0.1 & & -0.1 & 0.0 & \\
\hline Ot which: Contribution trom real GUF growth & -0.4 & -4.1 & -2.4 & & & -1.6 & -1.1 & -2.3 & -1.7 & -1.5 & -1.5 & & -1.4 & -1.1 & \\
\hline Contribution from real exchange rate depreciation & 5.3 & 1.9 & -3.6 & & & 12.5 & -7.6 & -0.9 & -0.1 & -0.1 & 0.2 & & $\ldots$. &... & \\
\hline Residual, including asset changes & -0.8 & 12.6 & 16.6 & & & 8.6 & 4.0 & 4.6 & 6.0 & 7.1 & 7.2 & & 6.1 & 5.2 & \\
\hline \multicolumn{16}{|l|}{ Other sustainability indicators } \\
\hline $\mathrm{PV}$ of public sector debt & 41.2 & 38.1 & 27.7 & & & 33.5 & 27.9 & 25.8 & 24.5 & 23.8 & 23.5 & & 23.2 & 18.6 & \\
\hline Ut which: roreign-currency denominated & 32.0 & 30.0 & 21.0 & & & 27.9 & 23.1 & 21.5 & 20.4 & 19.8 & 19.7 & & 19.9 & 16.2 & \\
\hline Of which: txternal & 32.0 & 30.0 & 21.0 & & & 27.9 & 23.1 & 21.5 & 20.4 & 19.8 & 19.7 & & 19.9 & 16.2 & \\
\hline PV of contingent liabilities (not included in public sector debt) & $\ldots$ & $\ldots$ & $\ldots$ & & & $\ldots$ & $\ldots$ & $\ldots$ & $\ldots$ & $\ldots$ & $\ldots$ & & $\ldots$ & $\ldots$ & \\
\hline Gross financing need $2 /$ & -0.1 & -10.6 & -14.5 & & & -7.9 & -1.9 & -2.6 & -4.4 & -4.7 & -4.7 & & -2.8 & -2.6 & \\
\hline PV of public sector debt-to-revenue and grants ratio (in percent) & 78.4 & 65.0 & 42.8 & & & 53.4 & 48.3 & 45.1 & 41.0 & 40.3 & 41.1 & & 42.2 & 33.8 & \\
\hline PV of public sector debt-to-revenue ratio (in percent) & 81.8 & 66.2 & 43.6 & & & 55.2 & 51.6 & 49.2 & 45.4 & 44.0 & 43.3 & & 43.2 & 34.6 & \\
\hline Ut which: External 31 & 63.4 & 52.1 & 33.0 & & & 45.9 & 42.7 & 41.0 & 37.8 & 36.7 & 36.3 & & 37.2 & 30.2 & \\
\hline Debt service-to-revenue and grants ratio (in percent) $4 /$ & 12.3 & 5.3 & 10.0 & & & 2.8 & 3.9 & 3.5 & 3.4 & 3.1 & 3.1 & & 2.7 & 2.9 & \\
\hline Debt service-to-revenue ratio (in percent) $4 /$ & 12.9 & 5.4 & 10.2 & & & 2.9 & 4.1 & 3.9 & 3.7 & 3.4 & 3.3 & & 2.8 & 2.9 & \\
\hline Primary deficit that stabilizes the debt-to-GDP ratio & -3.7 & -9.5 & -13.6 & & & -19.1 & 5.1 & -1.1 & -4.1 & -5.3 & -5.8 & & -3.9 & -3.5 & \\
\hline \multicolumn{16}{|l|}{ Key macroeconomic and fiscal assumptions } \\
\hline Real GDP growth (in percent) & 0.7 & 8.1 & 5.1 & 3.3 & 2.4 & 3.9 & 2.1 & 5.5 & 4.5 & 4.3 & 4.2 & 4.1 & 4.3 & 4.3 & 4.3 \\
\hline Average nominal interest rate on forex debt (in percent) & 2.0 & 1.4 & 10.8 & 3.1 & 2.7 & 0.8 & 1.2 & 1.3 & 1.4 & 1.5 & 1.5 & 1.3 & 1.6 & 1.8 & 1.6 \\
\hline Average real interest rate on domestic debt (in percent) & 2.3 & -3.1 & -3.2 & 4.8 & 14.4 & -1.9 & -1.5 & -1.6 & 0.4 & 0.1 & 0.5 & -0.7 & 0.3 & 0.3 & 0.3 \\
\hline Real exchange rate depreciation (in percent, + indicates depreciation) & 11.2 & 4.7 & -8.2 & 0.0 & 27.4 & 36.1 & & . & $\ldots$ & & & $\ldots$ & $\ldots$ & $\ldots$ & $\ldots$ \\
\hline Inflation rate (GDP deflator, in percent) & 4.3 & 8.6 & 9.2 & 7.4 & 3.1 & 9.6 & 8.7 & 8.2 & 5.4 & 5.0 & 3.8 & 6.8 & 4.0 & 4.0 & 4.0 \\
\hline Growth of real primary spending (deflated by GDP deflator, in percent) & 0.1 & 0.1 & 0.0 & 0.0 & 0.1 & 0.3 & 0.0 & 0.0 & 0.1 & 0.0 & 0.0 & 0.1 & 0.0 & 0.0 & 0.0 \\
\hline Grant element of new external borrowing (in percent) & $\ldots$ & $\ldots$ & $\ldots$ & $\ldots$ & $\ldots$ & 22.2 & 34.0 & 30.6 & 31.1 & 35.9 & 34.1 & 31.3 & 37.2 & 29.7 & $\ldots$ \\
\hline
\end{tabular}

Sources: Lesotho authorities; and Fund staff estimates and projections.

$1 /$ Covers gross debt of the general government.

2/ Gross financing need is defined as the primary deficit plus debt service plus the stock of short-term debt at the end of the last period.

$3 /$ Revenues excluding grants.

4/ Debt service is defined as the sum of interest and amortization of medium and long-term debt.

5/ Historical averages and standard deviations are generally derived over the past 10 years, subject to data availability. 


\section{Table 2b.Lesotho: Sensitivity Analysis for Key Indicators of Public Debt, Projections 2008-2028}

A. Alternative scenarios

A1. Real GDP growth and primary balance are at historical averages

A2. Primary balance is unchanged from 2008

A3. Permanently lower GDP growth 1/

A4.Permanent decline in SACU revenues by 6 percent of GDP starting in 2009 2/

$\begin{array}{rrrrrrrr}34 & 28 & 26 & 27 & 29 & 31 & 33 & 31 \\ 34 & 23 & 17 & 13 & 9 & 6 & \ldots & \ldots \\ 34 & 28 & 27 & 26 & 27 & 28 & 36 & 62 \\ 34 & 31 & 32 & 34 & 37 & 40 & 53 & 68\end{array}$

\section{B. Bound tests}

B1. Real GDP growth is at historical average minus one standard deviations in 2009-2010 B2. Primary balance is at historical average minus one standard deviations in 2009-2010

B3. Combination of B1-B2 using one half standard deviation shocks

B4. One-time 30 percent real depreciation in 2009

B5. 10 percent of GDP increase in other debt-creating flows in 2009

Baseline

A. Alternative scenarios

A1. Real GDP growth and primary balance are at historical averages

A2. Primary balance is unchanged from 2008

A3. Permanently lower GDP growth 1/

A4.Permanent decline in SACU revenues by 6 percent of GDP starting in 2009 2/

\section{B. Bound tests}

B1. Real GDP growth is at historical average minus one standard deviations in 2009-2010

B2. Primary balance is at historical average minus one standard deviations in 2009-2010

B3. Combination of B1-B2 using one half standard deviation shocks

B4. One-time 30 percent real depreciation in 2009

B5. 10 percent of GDP increase in other debt-creating flows in 2009

\section{Baseline}

\section{A. Alternative scenarios}

A1. Real GDP growth and primary balance are at historical averages

A2. Primary balance is unchanged from 2008

A3. Permanently lower GDP growth 1/

A4.Permanent decline in SACU revenues by 6 percent of GDP starting in 2009 2/

$\begin{array}{llllllll}34 & 28 & 30 & 31 & 32 & 34 & 43 & 53 \\ 34 & 36 & 42 & 41 & 40 & 39 & 36 & 27 \\ 34 & 32 & 35 & 35 & 35 & 36 & 40 & 40 \\ 34 & 36 & 34 & 32 & 31 & 30 & 28 & 22 \\ 34 & 37 & 34 & 33 & 32 & 32 & 30 & 23\end{array}$

PV of Debt-to-Revenue Ratio 3/

B. Bound tests

B1. Real GDP growth is at historical average minus one standard deviations in 2009-2010 B2. Primary balance is at historical average minus one standard deviations in 2009-2010

B3. Combination of B1-B2 using one half standard deviation shocks

B4. One-time 30 percent real depreciation in 2009

B5. 10 percent of GDP increase in other debt-creating flows in 2009

$\begin{array}{llllllll}53 & 49 & 52 & 51 & 54 & 60 & 79 & 95 \\ 53 & 63 & 74 & 68 & 67 & 69 & 66 & 49 \\ 53 & 55 & 61 & 58 & 60 & 63 & 72 & 73 \\ 53 & 63 & 58 & 53 & 52 & 53 & 50 & 40 \\ 53 & 64 & 60 & 55 & 54 & 55 & 54 & 41\end{array}$

Debt Service-to-Revenue Ratio 3/

Sources: Lesotho authorities; and Fund staff estimates and projections.

1/ Assumes that real GDP growth is at baseline minus one standard deviation divided by the length of the projection period

2/ In October 2008, SACU revenue projection was revised down by about 6 percent of GDP which is incorporated in the baseline scenario.

The alternative scenario assumes a permanent decline of the same magnitude relative to the baseline for the entire projection period starting in 2009

3/ Revenues are defined inclusive of grants. 
Figure 1. Lesotho: Indicators of Public and Publicly Guaranteed External Debt under Alternatives Scenarios, 2008-2028 1/
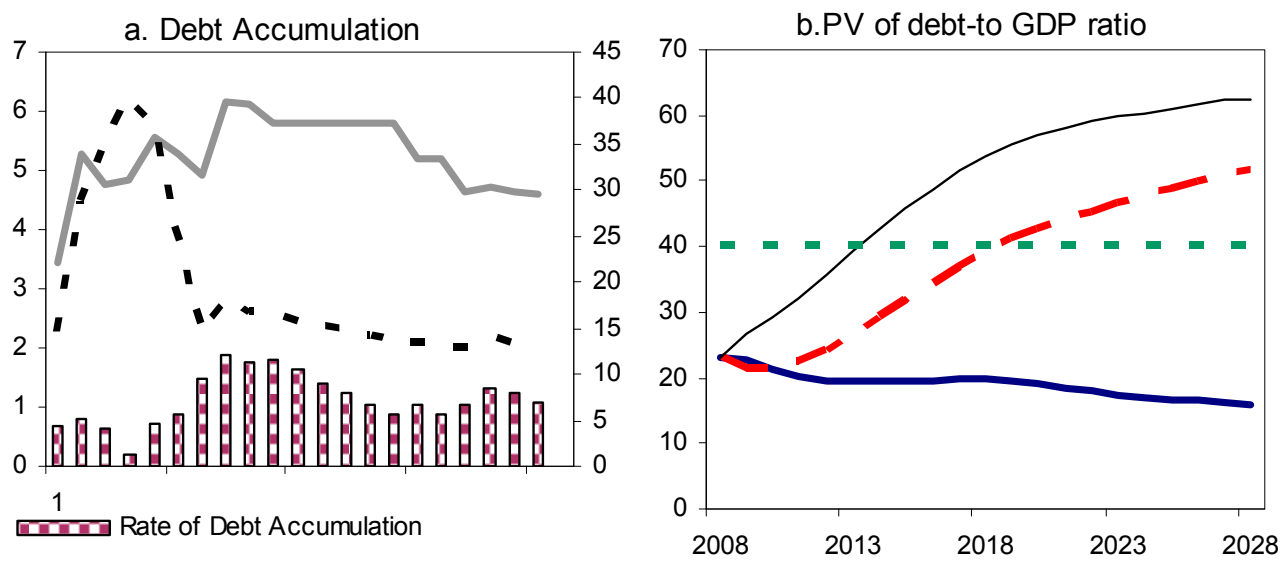

Grant element of new borrow ing (\% right
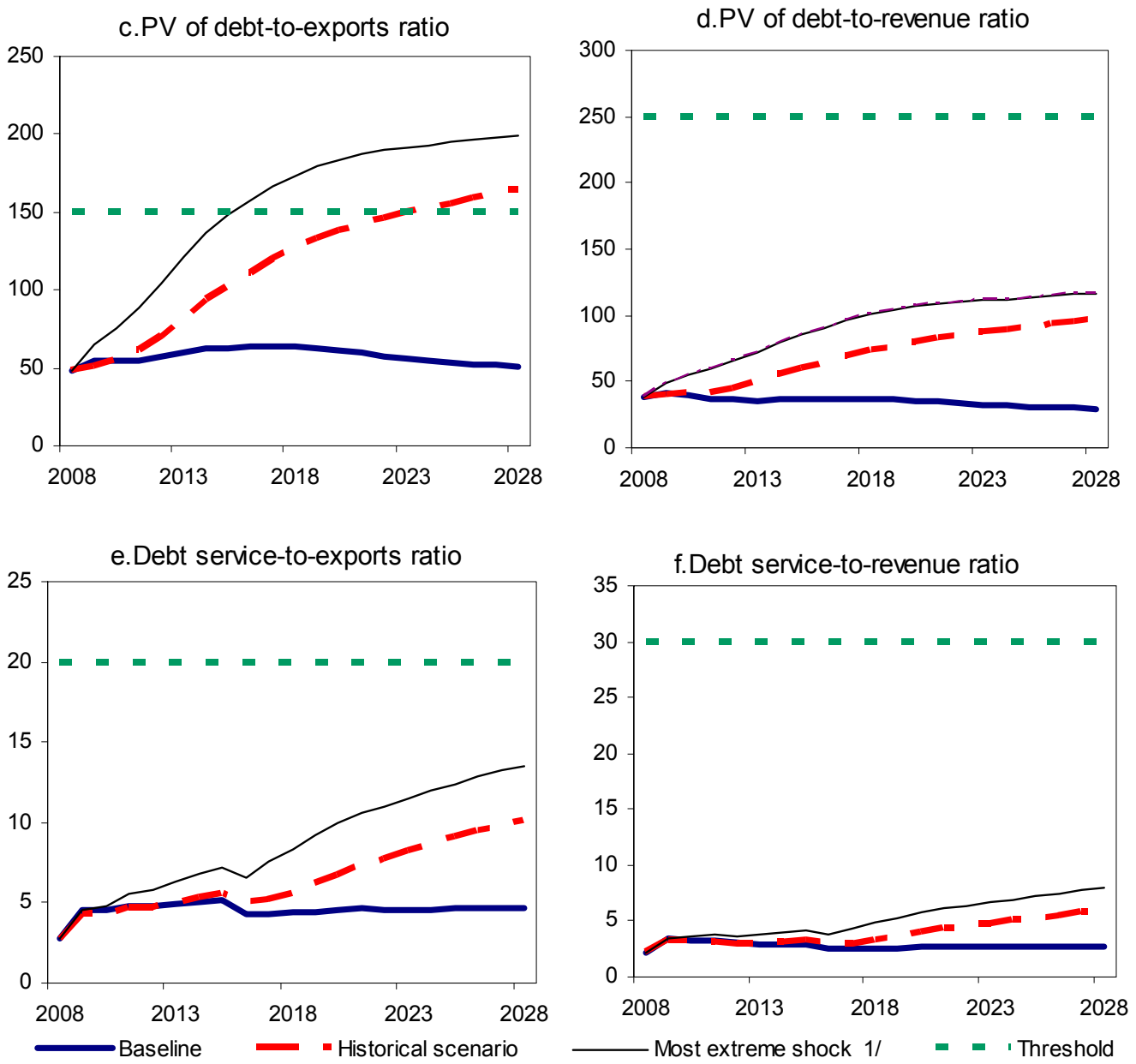

Source: Staff projections and simulations.

1/ The most extreme stress test is the test that yields the highest ratio in 2018. In all the panels it corresponds to a sustained reduction in SACU revenue. 
Figure 2 Lesotho: Indicators of Public Debt Under Alternative Scenarios, 2008-2028 1/
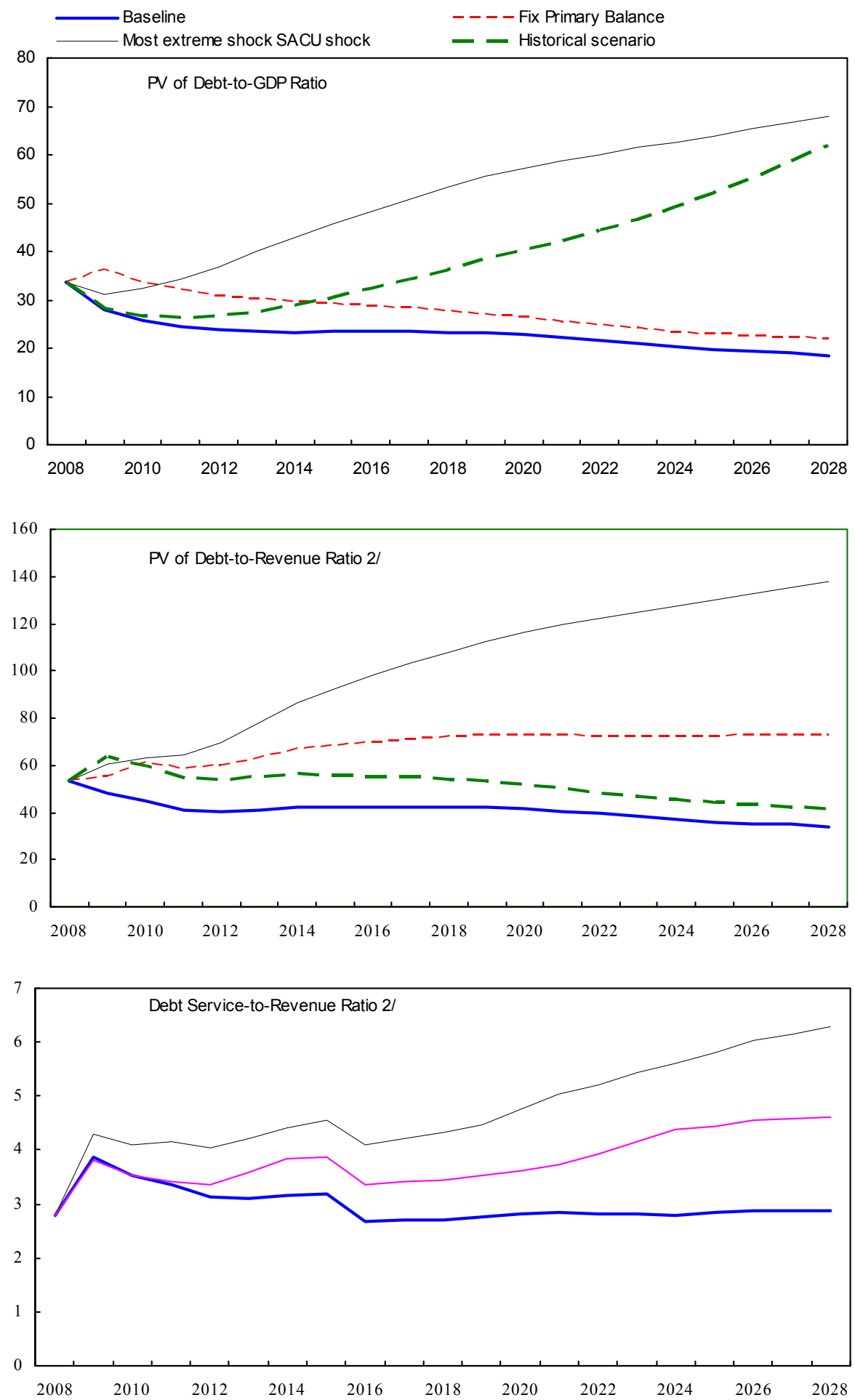

Sources: Lesotho authorities; and Fund staff estimates and projections.

1/ The most extreme stress test is the test that yields the highest ratio in 2018.

2/ Revenues are defined inclusive of grants. 


\section{INTERNATIONAL MONETARY FUND}

EXTERNAL

Public Information Notice

RELATIONS

DEPARTMENT

Public Information Notice (PIN) No. 09/132

International Monetary Fund

FOR IMMEDIATE RELEASE

$70019^{\text {th }}$ Street, NW

December 10, 2009

Washington, D. C. 20431 USA

\section{IMF Executive Board Concludes 2008 Article IV Consultation with the Kingdom of Lesotho}

On February 9, 2009, the Executive Board of the International Monetary Fund (IMF) concluded the Article IV consultation with the Kingdom of Lesotho. ${ }^{1}$

\section{Background}

Lesotho has made significant progress in macroeconomic performance but the pace of implementation of key structural reforms has been slow. After a decade of low growth, economic activity surged above historic trends, averaging 6.6 percent during 2006-07 driven by the mining, textile and construction sectors. However, poverty has seen only a modest decline, and the HIVIAIDS epidemic continues to impact negatively on the economy, public service delivery and social development, and threatens the achievement of the Millennium Development Goals (MDGs).

Large transfers from the Southern African Customs Union (SACU), together with workers' remittances and exports of textiles and diamonds, contributed to a sizable build-up of international reserves equivalent to 6.7 months of imports at end-2007. Inflation has surged from 6.4 percent at end-2006 to 11.8 percent at end-November 2008 owing mainly to the rise in food and fuel prices. Growth in broad monetary aggregates remains high, reflecting the continued accumulation of net foreign assets.

\footnotetext{
${ }^{1}$ Under Article IV of the IMF's Articles of Agreement, the IMF holds bilateral discussions with members, usually every year. A staff team visits the country, collects economic and financial information, and discusses with officials the country's economic developments and policies. On return to headquarters, the staff prepares a report, which forms the basis for discussion by the Executive Board. At the conclusion of the discussion, the Managing Director, as Chairman of the Board, summarizes the views of Executive Directors, and this summary is transmitted to the country's authorities.
} 
In 2007/08, Lesotho recorded its fifth consecutive fiscal surplus reflecting buoyant SACU receipts, increases in value-added and income taxes as well as underspending of both recurrent and development expenditure. Debt indicators have also improved as the use of SACU receipts to retire nonconcessional debt contributed to a decline in the ratio of external debt-to-GDP to 36 percent at the end of 2007 from 85.5 percent in 2002.

Financial sector vulnerabilities stem from the weakly supervised nonbank financial institutions and the potential re-emergence of Ponzi schemes. The banking sector thus far appears not to have been seriously affected by the current global financial crisis.

The medium term economic outlook is somewhat clouded in the wake of the global financial crisis and economic downturn. Downside risks remain from a potential further decline in SACU revenues, and lower exports of textiles and minerals should the global economic downturn be protracted.

\section{Executive Board Assessment}

Executive Directors commended the authorities for their prudent macroeconomic management, which has contributed to the recent strong economic performance and a continued build-up of international reserves. Directors noted, however, that Lesotho continues to face the challenges of sustaining economic growth, tackling widespread poverty and the high incidence of HIVIAIDS, and achieving the Millennium Development Goals. Addressing these challenges has been further complicated by the on-going global economic and financial crisis, which could result in a reduction in Southern African Customs Union (SACU) revenues, remittances, and exports of textiles and minerals.

Against this background, Directors emphasized the need for continued vigilance on the macroeconomic front, with a view to preserving fiscal and external stability. They also considered it crucial to fast-track the implementation of structural reforms, aimed particularly at promoting broad-based growth. In this regard, Directors welcomed the authorities' growth diagnostic study, which would guide the prioritization of efforts to remove constraints to growth. They stressed in particular the importance of land reform, which has the potential to reinvigorate the financial, tourism, and manufacturing sectors, critical for employment creation and sustained poverty reduction.

Directors called on the authorities to accelerate the pace of reforms, supported by the World Bank and the Millennium Challenge Corporation (MCC). Determined efforts to reduce the cost of doing business, improve the investment climate, and increase investment in human and physical capital would help consolidate the recent gains in growth performance and improve competitiveness. Directors welcomed the authorities' intention to set up a high-level committee that will ensure rapid implementation of these reforms.

Directors encouraged the authorities to adopt a fiscal strategy to mitigate risks from potentially lower SACU revenues, while creating fiscal space for increased social spending and growthenhancing investment. This includes strengthening non-SACU revenues and tax administration, 
and containing recurrent spending. Directors also saw the need to accelerate civil service reform and to contain the wage bill, including by linking future increases to performance and to qualified professionals within the health and education sectors.

Directors welcomed the authorities' intention to improve the process of public expenditure management. They supported the plan to formulate future budgets on the basis of the expected outturn, noting that this would make the budget a more effective tool for public policy, while improving transparency, accountability, and effective use of resources. Directors also underscored the importance of prudent debt policy, including seeking loans on highly concessional terms.

Directors agreed that Lesotho's monetary and exchange rate regime under the Common Monetary Area helps maintain price stability and facilitates capital and current transactions with the country's most important economic partner, South Africa. They emphasized, however, that maintaining external sustainability will require enhanced productivity and competitiveness, and pursuit of a prudent fiscal policy.

Directors noted that, while the banking sector has weathered the global financial crisis relatively well, more needs to be done to improve regulatory oversight and increase access to financial services for small- and medium-scale enterprises and the underserved population. In this regard, they welcomed the efforts underway, supported by the MCC and the International Fund for Agricultural Development, to deepen and enhance efficiency of the financial sector.

Directors welcomed the authorities' recent decision to close the largest Ponzi scheme, and looked forward to an early resolution. They urged that prompt action be taken to address vulnerabilities arising from the weakly supervised nonbank financial institutions, including by expeditiously amending the Financial Institution Act to strengthen the supervision and regulatory role of the central bank. Directors also supported the planned amendment of the Cooperative Societies Act, which would prohibit deposit mobilization from nonmembers of credit cooperatives. They welcomed the passage of the Anti-Money Laundering Law, and looked forward to the establishment of the Financial Intelligence Unit.

Public Information Notices (PINs) form part of the IMF's efforts to promote transparency of the IMF's views and analysis of economic developments and policies. With the consent of the country (or countries) concerned, PINs are issued after Executive Board discussions of Article IV consultations with member countries, of its surveillance of developments at the regional level, of post-program monitoring, and of ex post assessments of member countries with longer-term program engagements. PINs are also issued after Executive Board discussions of general policy matters, unless otherwise decided by the Executive Board in a particular case. 
Lesotho: Selected Economic and Financial Indicators, 2004-08 1/

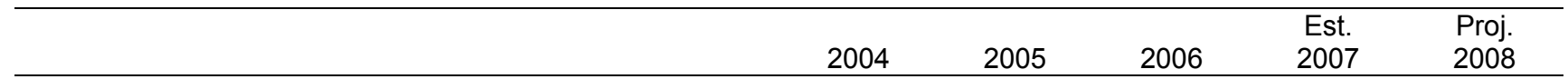

(Annual percent change; unless otherwise specified)

Real economy

Real GDO growth

GDP at current market prices (in millions of maloti)

Inflation (period average

$\begin{array}{rrrrr}4.6 & 0.7 & 8.1 & 5.1 & 3.9 \\ 8,332 & 8,750 & 10,269 & 11,778 & 13,418 \\ 5.0 & 3.4 & 6.1 & 8.0 & 12.0\end{array}$

(In percent of GDP)

\section{National accounts}

Gross domestic investment

Gross national savings

$\begin{array}{rrrrr}24.8 & 24.7 & 24.3 & 24.3 & 28.2 \\ 19.1 & 17.2 & 28.6 & 37.0 & 24.4 \\ & & & & \\ 50.5 & 52.6 & 58.7 & 64.8 & 62.7 \\ 47.8 & 50.4 & 57.6 & 63.5 & 60.7 \\ 2.7 & 2.2 & 1.1 & 1.3 & 2.0 \\ 44.7 & 47.6 & 45.9 & 48.3 & 53.9 \\ 3.1 & 2.8 & 11.7 & 15.2 & 6.8 \\ 5.8 & 5.0 & 12.7 & 16.5 & 8.9\end{array}$

\section{Central government}

Revenue and grants

Revenue

Total grants

Total expenditure and net lending

Overall balance (excluding grants)

Overall balance

(Annual percent change; unless otherwise specified)

\section{Money and credit}

Net domestic assets of the banking system 2/

Money and quasi-money (M2)

\begin{tabular}{rrrrr}
-19.1 & -3.3 & -37.9 & -49.7 & -36.5 \\
3.4 & 9.1 & 35.3 & 16.4 & 16.2 \\
\multicolumn{5}{c}{ (In percent of GDP) }
\end{tabular}

\section{External sector}

Current account balance

Excluding official transfers

Including official transfers

External public debt 3/

External debt-service ratio 4/

Gross official reserves (end of period)

In millions of U.S. dollars

In months of imports of goods and services

\begin{tabular}{rrrrr}
-24.2 & -28.6 & -20.2 & -24.8 & -37.5 \\
-5.7 & -7.5 & 4.3 & 12.7 & -3.7 \\
48.3 & 45.2 & 42.1 & 36.2 & 46.7 \\
8.2 & 10.0 & 6.4 & 5.0 & 2.8 \\
& & & & \\
458.2 & 501.4 & 692.6 & 957.7 & 981.9 \\
4.0 & 4.3 & 5.7 & 6.7 & 6.6 \\
& & & & \\
\hline
\end{tabular}

Sources: Lesotho authorities; and Fund staff estimates and projections.

1/ Fiscal year beginning in April. All fiscal data are reported on a calendar basis.

2/ Change in percent of M2 at the beginning of the period. Data for 2008 refer to end-September 2008.

$3 /$ The depreciation of the loti had a significant effect on the debt-to-GDP ratio in 2008.

4/ In percent of exports of goods and services.

Washington, D.C. 20431 • Telephone 202-623-7100 • Fax 202-623-6772 • www.imf.org 


\section{Statement by Moeketsi Majoro, Executive Director for the Kingdom of Lesotho February 9, 2009}

\section{Introduction}

On behalf of the Lesotho authorities, I would like to thank staff for the candid and productive discussions during the Article IV mission, and the Executive Board and Management for their continued support. The authorities value the advice proffered by the Fund and are in general agreement with the thrust of the reports.

Lesotho has made significant progress in achieving macroeconomic stability over the years. However, Lesotho remains susceptible to external shocks. Growth has been supported by the manufacturing and diamond mining sectors. Prudent use of fiscal surpluses to reduce public debt and build up reserves has enhanced fiscal and external stability. While the current global financial crisis has thus far had limited impact on Lesotho's banking system, the economy has been affected indirectly through reduced demand for exports, tightened trade credit for the textile industry which has links with Asian financial markets and significantly reduced investment flows for the diamond mining sector.

The authorities remain committed to the implementation of prudent policies to preserve macroeconomic stability and promote sustainable growth.

\section{Recent Economic Developments and Macroeconomic Outlook}

Lesotho's economic growth is estimated to have slowed down to 5.1 percent in 2007, from 7.2 percent in 2006, mainly due to a decline in agricultural output as a result of adverse weather conditions. Growth was largely supported by increased production in the mining and manufacturing sectors. The mining sector benefited from additional investment and efficiency gains in diamond mining as well as the favorable outlook on the international prices of precious stones. The manufacturing sector's contribution emanated mainly from textiles and clothing exports, which were mostly destined to the US. Inflation continued to rise throughout most of the last year, mainly as a result of high fuel and food prices and it reached 12 percent per annum in September 2008. However, it declined to 10.6 percent in December, as the international oil and food prices abated.

Fiscal performance remained strong in 2007/08, with government budgetary operations recording a surplus for the fifth consecutive year. The surplus, estimated at around 10 percent of GDP, was supported by the increased SACU and domestic tax revenues as well as lower expenditures. Surpluses continued to be realized during the first half of 2008/09 fiscal year. The external position also improved, resulting in the accumulation of foreign reserves to about 7 months of import cover by the end of 2007. The improvement in the balance of payments position largely reflected increased SACU receipts as well as a rise in foreign direct investment in manufacturing and mining industries. 
Medium term economic prospects seem to be mixed. Growth is expected to benefit from the recovery of the agricultural sector and increased construction activity due to commencement of new public infrastructure projects. Investment projects to be implemented under the Millennium Challenge Corporation (MCC) compact and implementation of Phase II of the Lesotho Highlands Water Project are expected to contribute significantly to growth. Prudent macroeconomic policies are expected to continue to support macroeconomic stability.

Downside risks include the negative impact of unfavorable developments in the external environment and the persistent drought on agriculture. The manufacturing sector is expected to be adversely affected by the reduction in demand in the US economy, as the recession intensifies and credit conditions tighten in Asia, where most of the firms source their financing, while the diamond mining sector could be adversely affected by the falling international prices of rough diamonds. Developments in the global regime for textiles, such as the erosion of trade preferences, would also add strain to Lesotho's export sector. Inflationary pressures are expected to continue to follow developments in international oil and food prices. The slowdown in the South African economy is also expected to have negative fiscal and external effects on Lesotho's economy through declining SACU revenues and export demand. The authorities are, however taking necessary measures to help address these challenges.

Government is looking at long term measures such as irrigation to mitigate the problem of persistent droughts.

\section{Policies}

\section{Fiscal Policy}

The authorities remain committed to prudent macroeconomic management and consolidation of macroeconomic stability that has been achieved over the last years. Prudent fiscal policy will continue to be the main tool for macroeconomic management and will be aimed at ensuring sustainable fiscal and external stability. Debt sustainability and external stability has improved significantly over the years, as government used the fiscal surpluses to reduce nonconcessional public debt.

Going forward, SACU revenues are expected to decline as a result of the reduction in the common revenue pool cause by the slowdown in the South African economy and the effects of trade liberalization on customs revenues. This poses a significant challenge to fiscal policy given that SACU revenues constitute the bulk of government revenues. In this regard, the authorities will continue their efforts to bolster domestic revenue collections mainly through measures to improve revenue administration. Some expenditure restraint, consistent with the projected fall in SACU revenues while at the same time providing for the infrastructure and social needs of the country, will also be exercised. Fiscal surpluses are expected to continue to be realized, albeit at narrower levels, in the medium term. 
Significant progress is being made in strengthening public financial management (PFM). PFM reforms which were initiated in 2005 include the review of procurement regulations, introduction of the medium term expenditure framework (MTEF) and the introduction of the Integrated Financial Management Information System (IFMIS). Implementation of the new procurement regulations began in 2007 and the process of implementing IFMIS is at an advanced stage. The IFMIS system is already in place and will start operating during the coming budget year (2009/10). The IFMIS is expected to enhance all the accounting aspects of the budget process, including commitment control and generation of reports for budget monitoring.

The authorities are also putting measures in place to improve budgeting in order to improve the effectiveness of the budget as an instrument to achieve the national development objectives and to enhance the efficiency of resource allocation among ministries. In this connection the MTEF, which had been introduced on a pilot basis, has now been rolled out to all government ministries and its functionality is now being expanded. New Public Financial Management and Accountability and Audit bills have been drafted to underpin the PFM reforms and are currently undergoing review.

Public service effectiveness will be addressed under the Public Sector Improvement and Reform Program (PSIRP), which is undergoing some design. It is intended to cover civil service reform and decentralization of public services.

Lesotho's public and publicly guaranteed debt is sustainable, although there is a moderate risk of debt distress. To safeguard debt sustainability, the authorities will continue to reduce the amount of commercial debt outstanding and to the extent possible, limit borrowing to concessional terms.

\section{Monetary Policy and Financial Sector Issues}

Lesotho's CMA membership, which provides for the loti to be pegged at par to the rand and for free movement of capital within the CMA, has supported price stability. Monetary policy in Lesotho continues to be aimed at maintaining a strong external reserve position to support the exchange rate parity with the South African rand as well as to meet the country's external obligations. This has been supported by prudent fiscal policies.

The current global financial crisis has thus far had limited impact on Lesotho's banking system. The banking sector remains strong, with banks being profitable, well-capitalized, and liquid with relatively moderate and well provisioned non-performing loans (NPLs). Downside

risks posed by the relatively high concentration of the banks' portfolios on a few borrowers are mitigated by the full collateralization of most of the loans to the top twenty borrowers.

Financial intermediation has continued to be limited as banks remain risk averse and as a result access to financial services is lacking for the bulk of the population. The authorities are making efforts to increase access to financial services, with the support of the MCC and under 
the International Fund for Agricultural Development (IFAD) rural financial intermediation program (RUFIP). These include the strengthening of the institutional and operational framework of the Lesotho Postbank, and the development of the microfinance sector. The improvement of financial intermediation would also be facilitated by the creation of the credit reference bureau, development of a national identity system, an Automatic Clearing House and modernization of the commercial court. The ongoing review of legislation for the non-bank financial institutions (NBFIs), under RUFIP, is expected to usher a sound regulatory framework for NBFIs, which pose a challenge to the ability of the authorities to supervise and regulate as they are not covered by the current financial institutions laws.

The new Anti-Money Laundering legislation has been approved by Parliament and preparations are underway to develop regulations and to establish a Financial Intelligence Unit.

\section{Growth Policies}

The Government's key priority remains that of promoting sustainable economic growth to reduce poverty and move towards achieving the MDGs. The authorities have developed a draft Growth Strategy Paper (GSP), which identifies opportunities and binding constraints on growth. Going forward, the authorities plan to devote significant resources in the medium term fiscal framework and the annual budgets, as well as the Government's reform initiatives with the priorities identified in the GSP.

To address the constraints on growth, the authorities are, with the support of the World Bank and the MCC, undertaking reforms to improve the investment climate and international competitiveness. Effective implementation of the MCC's private sector development component and the World Bank's ongoing Private Sector Competitiveness and Economic Diversification Program would help reduce the cost of doing business in Lesotho. These would include addressing the legal and regulatory constraints, improving private sector access to credit and piloting economic diversification initiatives in tourism and agribusiness.

A trade and investment facilitation centre has already been established to serve as a one stop shop for potential investors and to reduce regulatory impediments and facilitate investment. A modernized Companies Bill is under preparation to support these business reforms.

\section{Conclusion}

In conclusion, I wish to reaffirm the authorities' commitment to the prudent macroeconomic management which has facilitated the achievement of macroeconomic stability. Promotion of growth and poverty reduction also remain high in their policy agenda. They are confident that, with the continued support of the Fund and the international community, they will make meaningful progress toward achieving their developmental goals. 